University of Tennessee Health Science Center

UTHSC Digital Commons

\title{
Role of SMARCE1 and BHLHE40 in Breast Cancer Metastasis
}

Aarti Sethuraman

University of Tennessee Health Science Center

Follow this and additional works at: https://dc.uthsc.edu/dissertations

Part of the Biological Phenomena, Cell Phenomena, and Immunity Commons, Medical Cell Biology Commons, Medical Molecular Biology Commons, and the Neoplasms Commons

\section{Recommended Citation}

Sethuraman, Aarti (http://orcid.org/https://orcid.org/0000-0002-7349-7586), "Role of SMARCE1 and BHLHE40 in Breast Cancer Metastasis" (2018). Theses and Dissertations (ETD). Paper 454. http://dx.doi.org/10.21007/etd.cghs.2018.0450.

This Dissertation is brought to you for free and open access by the College of Graduate Health Sciences at UTHSC Digital Commons. It has been accepted for inclusion in Theses and Dissertations (ETD) by an authorized administrator of UTHSC Digital Commons. For more information, please contact jwelch30@uthsc.edu. 


\section{Role of SMARCE1 and BHLHE40 in Breast Cancer Metastasis}

\section{Document Type}

Dissertation

Degree Name

Doctor of Philosophy (PhD)

\section{Program}

Biomedical Sciences

Research Advisor

Meiyun Fan, M.D., Ph.D.

\section{Keywords}

BHLHE40 / DEC1, Breast cancer metastasis, HBEGF, HIF1A, PTK2 / FAK, SMARCE1 / BAF57

\section{Subject Categories}

Biological Phenomena, Cell Phenomena, and Immunity | Diseases | Medical Cell Biology | Medical Molecular Biology | Medical Sciences | Medicine and Health Sciences | Neoplasms 


\title{
Role of SMARCE1 and BHLHE40 in Breast Cancer Metastasis
}

\author{
A Dissertation \\ Presented for \\ The Graduate Studies Council \\ The University of Tennessee \\ Health Science Center
}

\author{
In Partial Fulfillment \\ Of the Requirements for the Degree \\ Doctor of Philosophy \\ From The University of Tennessee
}

By

Aarti Sethuraman

May 2018 
Chapter 2 (C) 2016 by Biomed Central Ltd All other material (C) 2018 by Aarti Sethuraman. All rights reserved. 


\section{DEDICATION}

This is dedicated to my family and friends without their support, encouragements and firm faith in me this journey would not be possible. 


\section{ACKNOWLEDGEMENTS}

I would like to thank my graduate advisor, Dr. Meiyun Fan and my advisory Committee: Dr. Kui Li, Dr. Tiffany Seagroves, Dr. Lawrence Pfeffer and Dr. Zhao-hui $\mathrm{Wu}$ for their instrumental comments, ideas, support and motivation. I would also like to acknowledge Raya Krutilini and Martin Brown for their helpful assistance in projects presented here. I would also like to thank Jo Megan Garner, Martin Brown and Debolina Ganguly for providing me with daily scientific and comforting senseless discussions. Furthermore, I would like to recognize the Molecular Research Core (MRC) for their timely results and assistance. Finally, I would like to thank all the faculty and staff of the University of Tennessee Health Science Center, Center for Cancer Research for providing a friendly environment for learning and research. 


\begin{abstract}
Metastatic breast cancer (MBC) is mainly responsible for breast cancer-related deaths worldwide. MBC is currently untreatable and has a 5-year survival rate of as low as $26 \%$ in patients. Hypoxia has been identified as a driving force for distant metastasis in breast cancer. Hypoxic response is primarily mediated by hypoxia-inducible factor, HIF1A which recruits chromatin remodelers and activates target genes directly or via downstream effector transcription factors. Several accessory molecules have also been implicated to be involved in the hypoxic response elicited by tumor cells. In this study, we focus on two such transcriptional regulators, SMARCE1 and BHLHE40, which play a pro-metastatic role in breast cancer progression in-vivo.

SMARCE1 is a subunit of the chromatin remodeler complex SWI/SNF which causes nucleosome remodeling to facilitate expression of target genes. While aberrant activation of the chromatin-remodeling SWI/SNF complexes has been associated with cancer development and progression, the role of each subunit in tumor cells is poorly defined. This study characterizes the role of SMARCE1/BAF57 in regulating metastasis of breast cancer cells via the PTK2 pathway. SMARCE1-knockdown reduced lung metastasis of breast cancer cells and sensitized tumor cells to anoikis. In response to loss of attachment, SMARCE1 is shown to interact with and potentiate transcriptional activity of HIF1A, resulting in rapid PTK2 activation. Both HIF1A and PTK2 were indispensable for SMARCE1-mediated protection against anoikis by promoting activation of MAPK and AKT pathways while suppressing expression of pro-apoptotic BIM protein. Expression data analysis of a large cohort of human breast tumors revealed high expression of SMARCE1 or PTK2 is associated with poor prognosis and tumor relapse, and PTK2 expression is positively correlated with SMARCE1 expression in basal-like and luminal B subtypes of breast tumors.
\end{abstract}

BHLHE40 is a validated target of HIF1A and its expression level is correlated with breast cancer grade and progression in patients. However, its exact role in the metastatic process is unclear. Here we reveal a novel role of BHLHE40 in regulating hypoxia-induced secretion of HBEGF to promote tumor cell growth and metastasis. BHLHE40 knockdown significantly reduced primary tumor growth and lung metastasis in orthotopic xenograft and experimental metastasis models of breast cancer. Gene expression analysis implicated a role of BHLHE40 in transcriptional activation of HBEGF, a heparin binding epidermal growth factor. Chromatin immunoprecipitation (ChIP) and co-immunoprecipitation (CoIP) assays revealed that BHLHE40 induces HBEGF transcription by blocking DNA binding of HDAC1 and HDAC2. Cell-based assays showed that HBEGF is secreted through exosomes and acts to promote cell survival and migration. Public database provided evidence linking high expression of BHLHE40 and HBEGF to poor prognosis of triple negative breast cancer.

In summary, this study focused on two novel pro-metastatic mechanisms employed by HIF1A in breast cancer cells, which can be manipulated to target and modulate metastatic breast cancer therapeutically. While SMARCE1 plays an essential 
role in breast cancer metastasis by protecting cells against anoikis through the HIF1A/PTK2 pathway, BHLHE40 increases metastatic potential by regulating exosomal secretion of HBEGF, which promotes breast cancer cell survival and migration. 


\section{TABLE OF CONTENTS}

CHAPTER 1. INTRODUCTION .....................................................................................1

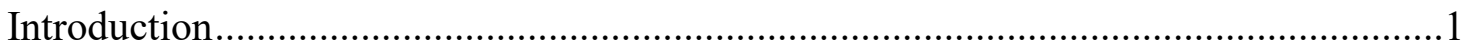

Prerequisites for Metastasis ............................................................................. 1

Role of Tumor Hypoxia in Metastasis ........................................................................

Hypoxia Inducible Factor HIF1A Drives Metastasis ................................................

Screening for Transcriptional Regulators Involved in Metastasis of Breast Cancer

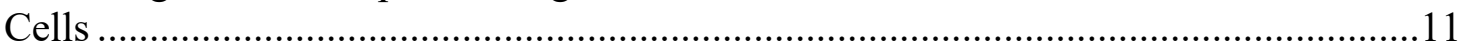

BHLHE40 and SMARCE1 Knockdown Reduces Lung Metastasis of Breast Cancer

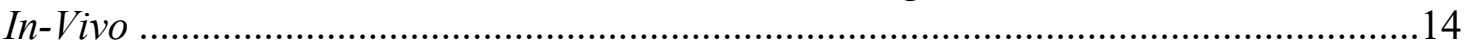

\section{CHAPTER 2. ROLE OF SMARCE1 IN METASTATIC BREAST CANCER ........17}

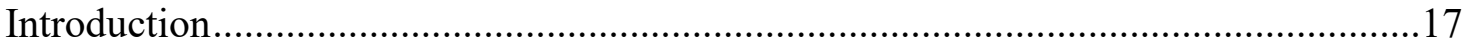

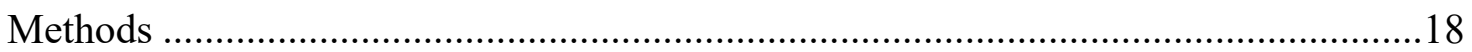

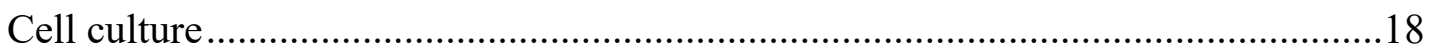

Orthotopic xenograft model and experimental lung metastasis model (tail-vein

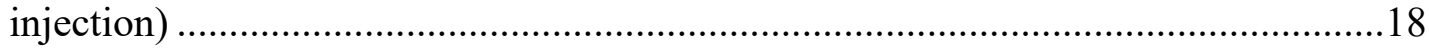

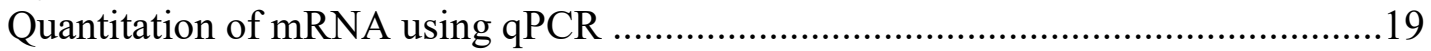

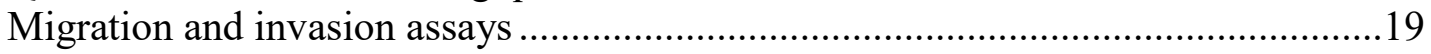

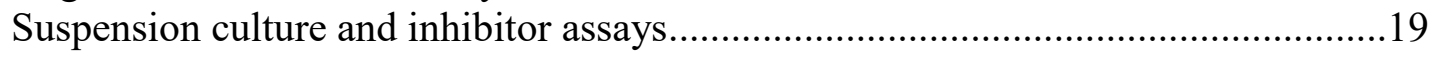

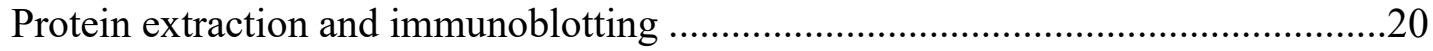

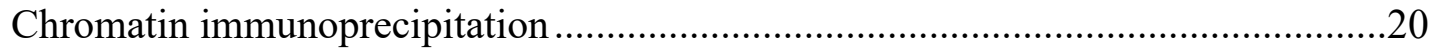

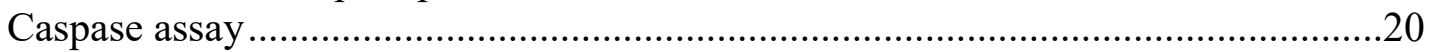

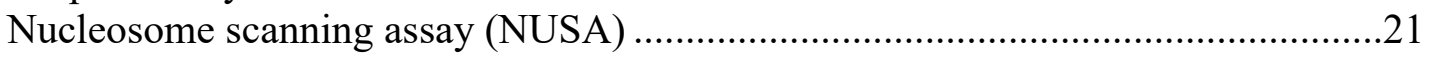

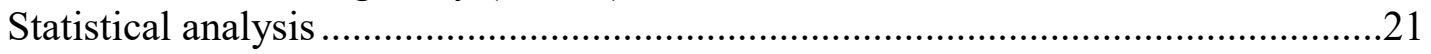

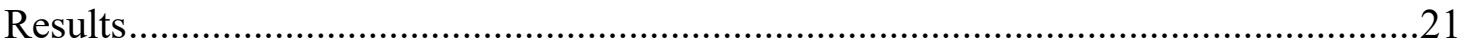

SMARCE1 knockdown reduces lung metastasis of breast cancer in vivo.................21

SMARCE1 knockdown reduces lung colonization of tumor cells inoculated

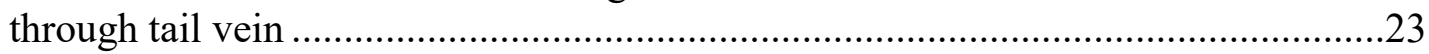

SMARCE1 knockdown sensitizes tumor cells to anoikis.......................................23

SMARCE1-mediated anoikis resistance requires activation of PTK2, MAPK and

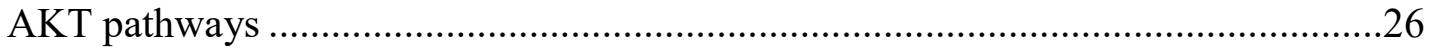

SMARCE1 collaborates with HIF1A to activate PTK2 transcription in detached cells ..........................................................................................................29

HIF1A activates $P T K 2$ transcription and downstream activation of survival

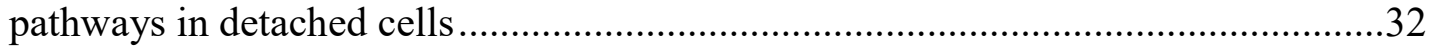

SMARCE1 knockdown sensitizes non-tumorigenic mammary epithelial cells to

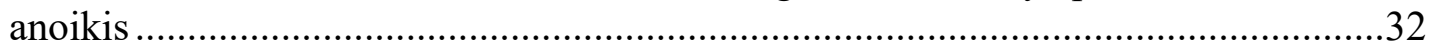

Higher SMARCE1 and PTK2 expression is associated with poor prognosis of patients with basal-like or luminal B subtype of breast tumors ................................36 Genomic alterations contribute to deregulation of SWI/SNF complexes in breast

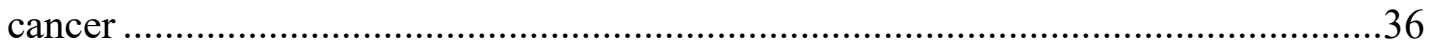

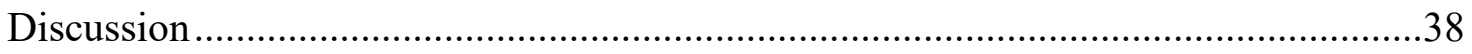


CHAPTER 3. ROLE OF BHLHE40 IN BREAST CANCER METASTASIS............40

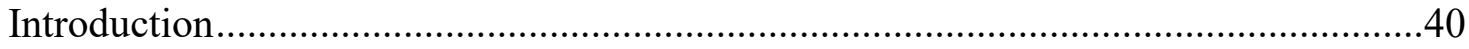

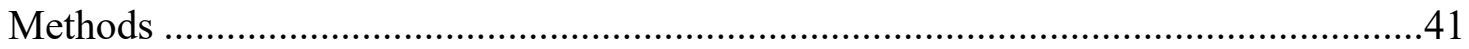

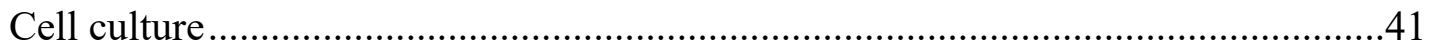

Orthotropic xenograft and experimental lung metastasis models ...............................41

Migration, invasion and wound healing assays …………….................................42

Suspension culture, viable cell counting and caspase assays....................................42

Luciferase reporter assay for HIF activity ............................................................42

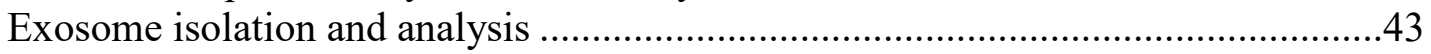

Gene expression microarray and qPCR analysis ......................................................43

Protein extraction, co-immunoprecipitation and immunoblotting..............................44

Chromatin immunoprecipitation ......................................................................44

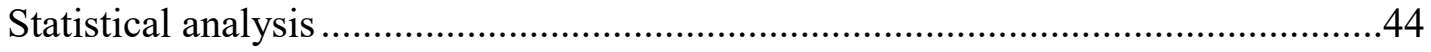

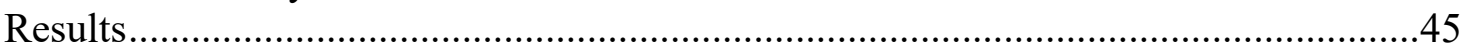

BHLHE40 knockdown leads to decreased primary tumor growth and lung metastases

BHLHE40 knockdown reduces lung colonization of tumor cells inoculated

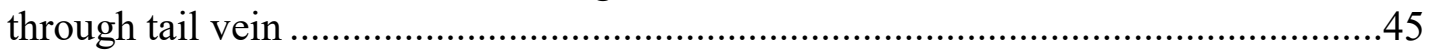

BHLHE40 acts to promote cell migration, invasion and survival .............................48

BHLHE40 is required for transcription activation of a set of cytokines and growth factors ..................................................................................................5

BHLHE40 activates HBEGF transcription by sequestering HDAC1 and HDAC2

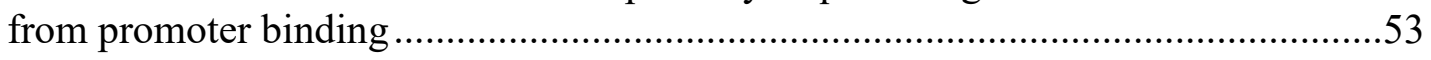

HBEGF acts to promote cell survival and migration.................................................53

High expression of BHLHE40 and HBEGF is associated with poor prognosis of

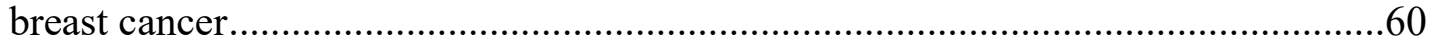

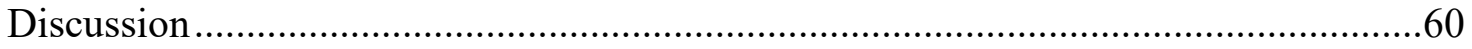

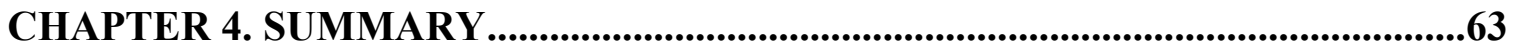

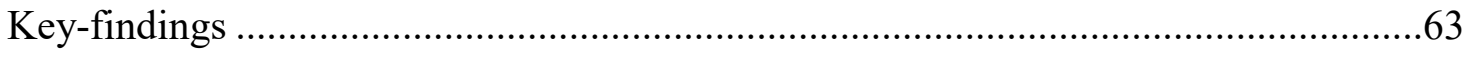

SMARCE1: Implications in Other Cancers and Further Direction ...............................66

BHLHE40: Implications in Other Cancers and Further Direction ...............................67

LIST OF REFERENCES .................................................................................68

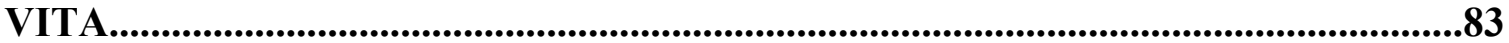




\section{LIST OF TABLES}

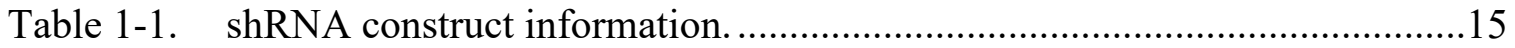

Table 1-2. Mean percentage of metastatic foci in-vivo. ...............................................16

Table 3-1. Correlated expression of BHLHE40 and its putative targets in breast

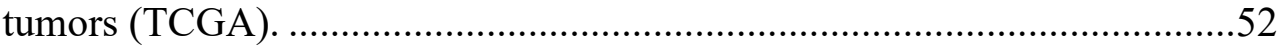

Table 4-1. Summary of epigenetic factors involved in hypoxia-regulated gene

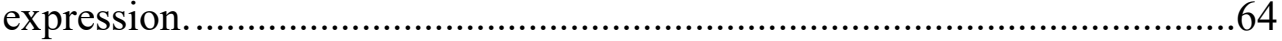




\section{LIST OF FIGURES}

Figure 1-1. Breast cancer metastasis. .....................................................................2

Figure 1-2. Metastatic cascade and signaling pathways involved..................................

Figure 1-3. Cell intrinsic and extrinsic pressures that drive metastasis..........................5

Figure 1-4. Transcriptional targets of HIF.......................................................

Figure 1-5. HIF1A inhibitors modulate different aspects of the HIF1A activation

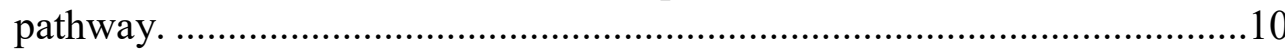

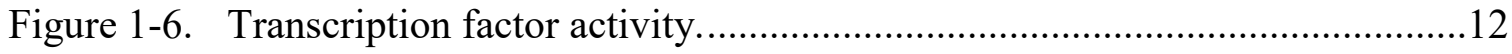

Figure 1-7. Orthotropic Xenograft model studies..................................................... 15

Figure 1-8. Fluorescent Lung metastases. ...................................................... 16

Figure 2-1. SMARCE1 knockdown reduces lung metastasis of breast cancer in vivo....22

Figure 2-2. SMARCE1 knockdown reduces lung colonization of tumor cells inoculated through tail veins.

Figure 2-3. SMARCE1 knockdown promotes cell migration, but sensitize cells to anoikis.

Figure 2-4. SMARCE1-mediated anoikis resistance requires activation of PTK2, MAPK and AKT pathways.

Figure 2-5. SMARCE1-dependent activation of PTK2, MAPK and AKT pathways by cell detachment.

Figure 2-6. SMARCE1 collaborates with HIF1A to activate PTK2 transcription in detached cells.

Figure 2-7. SMARCE1 orchestrates chromatin remodeling of PTK2 promoter in detached cells.

Figure 2-8. HIFIA activates PTK2 transcription and downstream survival pathways in detached cells.

Figure 2-9. PTK2 activation is essential for SMARCE1-mediated anoikis resistance in breast cancer cells.

Figure 2-10. Blocking SMARCE1 expression sensitizes non-tumorigenic mammary epithelial cells to anoikis. 
Figure 2-11. Higher SMARCE1 and PTK2 expression is associated with poor prognosis of breast cancer patients

Figure 3-1. BHLHE40 knockdown (KD) significantly reduced primary tumor size and lung metastatic burden in an orthotopic xenograft model.

Figure 3-2. BHLHE40 knockdown (KD) reduced lung colonization of tumor cells inoculated into the circulation via tail veins.

Figure 3-3. BHLHE40 depletion impaired cell migration, invasion and survival.

Figure 3-4. BHLHE40 knockdown reduced hypoxia-induced expression of a panel of cytokines and growth factors.

Figure 3-5. Exosomic secretion of HBEGF was reduced by BHLHE40 depletion in MDA-MB231 or TR cells.

Figure 3-6. BHLHE40 activates gene expression by sequestering HDAC1 and HDAC2 from genome DNA binding in MDA-MB-231 cells exposed to hypoxia and low glucose $\left(1 \% \mathrm{O}_{2} / \mathrm{LG}, 4 \mathrm{~h}\right)$.

Figure 3-7. BHLHE40 depletion reduced phosphorylation of EGFR, while increased Caspase 9 cleavage, in cells exposed to glucose depletion and hypoxia $\left(1 \% \mathrm{O}_{2} / \mathrm{GF}\right)$.

Figure 3-8. HIF-BHLHE40-HBEGF axis plays a role in promoting cell migration during wound healing.

Figure 3-9. Effect of BHLHE40 overexpression on molecular and phenotypic changes caused by HIF1A/EPAS1 double knockout (HIF-dKO) in MDA-MB-231 cells.

Figure 3-10. High expression of BHLHE40 and HBEGF is associated with poor prognosis of breast cancer.

Figure 4-1. Summary of results. 65 


\section{LIST OF ABBREVIATIONS}

\begin{tabular}{|c|c|}
\hline$\mu \mathrm{g}$ & Microgram \\
\hline$\mu \mathrm{M}$ & Micromolar \\
\hline $\mathrm{AKT} / \mathrm{Pi} 3 \mathrm{~K}$ & Phosphoinositide-3-kinase B \\
\hline ALDH & Aldehyde dehydrogenase \\
\hline ANGPTL4 & Angiopoietin like protein 4 \\
\hline BCL2 & B-cell lymphoma 2 \\
\hline BHLHE40 & Basic helix-loop-helix family member E40 \\
\hline BHLHE41 & Basic helix-loop-helix family member E41 \\
\hline BIM/BCL2L11 & BCL2 interacting mediator of cell death \\
\hline $\mathrm{BMF}$ & BCL2 modifying factor \\
\hline BT549 & Triple negative breast cancer cell line \\
\hline CCL & $\mathrm{C}-\mathrm{C}$ motif chemokine ligand \\
\hline $\mathrm{CD} 24$ & Small cell lung carcinoma cluster 4 antigen \\
\hline CD274 & Programmed death ligand 1 \\
\hline CD44 & Hematopoietic cell E- and L-selectin ligand \\
\hline CDH1 & E-cadherin \\
\hline $\mathrm{CDH} 2$ & N-cadherin \\
\hline CHIP & Chromatin immunoprecipitation \\
\hline CLOCK/BMAL1 & Cicardian locomoter output cycles kaput gene, clock family gene \\
\hline CoIP & Co-immunoprecipitation \\
\hline CREB & cAMP responsive element binding protein \\
\hline CREBBP & CREB binding protein \\
\hline
\end{tabular}




\begin{tabular}{ll} 
CSF1/M-CSF & Colony stimulating factor 1 \\
CTGF & Connecting tissue growth factor \\
CXCL & C-X-C motif chemokine ligand \\
CXCR & C-X-C motif chemokine receptor \\
DFMS & Distant metastasis free survival \\
dsDNase & Double strand specific DNase \\
E2F & Transcription factor in eukaryotes \\
ECM & Extracellular matrix \\
EGF & Epidermal growth factor \\
EGFR & Epidermal growth factor receptor \\
EHMT2 & Eukaryotic histone lysine methyltransferase 2 \\
EMT & Epithelial Mesenchymal Transition \\
EP300 & E1A binding protein P300 \\
EPAS1/HIF2A & Endothelial PAS domain, hypoxia inducible factor 2 alpha \\
ERBB2/HER2 & Human epidermal growth factor receptor 2 \\
EV & Empty vector \\
FACS & Flourescence-activated cell sorting \\
FOS & FBJ murine osteosarcoma viral proto-oncogene \\
H & Time in hours \\
HBEarin binde negating epidermal growth factor & \\
HC & Heacetylase \\
\hline
\end{tabular}




\begin{tabular}{|c|c|}
\hline HIF & Hypoxia inducible factor \\
\hline HIF $1 \mathrm{~A} / \mathrm{HIF} 1 \alpha$ & Hypoxia inducible factor 1 alpha subunit \\
\hline $\mathrm{HIF}-\mathrm{dKO}$ & HIF1A and EPAS1 double knockout clone \\
\hline IL & Interleukins \\
\hline ISWI & Imitation SWI chromatin remodeler \\
\hline ITGA & Integrin alpha subunit \\
\hline JNK & c-Jun N-terminal kinases \\
\hline KD & Knockdown \\
\hline KDM6B & Lysine-specific demethylase 6B \\
\hline $\mathrm{KO}$ & Knockout \\
\hline KRAS & C-kristen-Ras proto-oncogene, GTPase \\
\hline L1CAM & L1 cell adhesion molecule protein \\
\hline LDHA & Lactate dehydrogenase \\
\hline LM & Lung metastatic cellline derived from MDA-MB-231 \\
\hline LOX & Lysyl oxidase \\
\hline MAPK/ERK & Mitogen activated protein kinase \\
\hline $\mathrm{MBC}$ & Metastatic breast cancer \\
\hline MDA-MB-231 & Triple negative breast cancer cell line (MB231) \\
\hline MEGM & Mammary epithelial cell growth medium \\
\hline MEM & Minimal essential medium \\
\hline $\mathrm{ml}$ & Milliliter \\
\hline $\mathrm{mm}$ & Millimeter \\
\hline MMP & Matrix metalloprotease \\
\hline
\end{tabular}




$\begin{array}{ll}\text { MMTV-PyMt } & \text { Transgenic mice model } \\ \text { MYC } & \text { Myc proto-oncogene } \\ \text { NF-kB } & \text { Nuclear factor kappa B subunit } \\ \text { nm } & \text { Namometer } \\ \text { NSG } & \text { NOD Cg-Prkdcscis Il2rgtm1Wjl/SzJ mice model } \\ \text { NUSA } & \text { Nucleosome scanning assay } \\ \text { NYF } & \text { Nuclear transcription factor } \\ \text { PDGF } & \text { Platelet-derived growth factor } \\ \text { PD-L1 } & \text { Programmed cell death ligand 1 } \\ \text { PHD } & \text { Prolyl dehydrogenase } \\ \text { pO } 2 & \text { Partial pressure of oxygen } \\ \text { PTK2/FAK } & \text { protein tyrosine focal adhesion kinase } \\ \text { qPCR } & \text { Quantitative polymerase chain reaction } \\ \text { RANKL } & \text { Receptor activator of nuclear factor kappa B ligand } \\ \text { RFS } & \text { Relapse free survival } \\ \text { RIPA } & \text { Radioimmunoprecipitation assay buffer } \\ \text { ROS } & \text { Reactive oxygen species } \\ \text { sd } & \text { Standard deviation } \\ \text { SDS-PAGE } & \text { short hairpin RNA } \\ \text { shRNA } & \text { Short inhibitory RNA } \\ \text { siRNA } & \text { BARCA2/BRM } \\ \text { SMAR/BRGe, ATPase subunit of SWI/SNF }\end{array}$


SMARCE1/BAF57 BRG1 associated factor 57, SWI/SNF subunit

SNAI Snail family zinc finger transcription factor

STAT Signal transducer and activator of transduction family protein

SWI/SNF Switching defective sucrose non-fermenting complex

TCF3 Transcription factor 3

TCGA The cancer genome atlas

TFGB/TGF $\beta \quad$ Transforming growth factor beta

TNBC Triple negative breast cancer

TNFSF11 Tumor necrosis factor superfamily, member 11

TP53 Tumor protein 53

TR Tamoxifen resistant cell line derived from MCF7 cell line

TWIST Twist family basic helix-loop-helix transcription factor

U Units

VEGF Vascular endothelial growth factor

ZEB Zinc finger E-box binding homeobox protein 


\section{CHAPTER 1. INTRODUCTION}

\section{Introduction}

Metastasis is defined as the development of secondary malignant lesions distant from the primary site of origin. Breast cancer commonly metastasizes to the lung, liver, bones, and brain. Clinically metastatic breast cancer, known as stage IV (advanced stage) or recurrent cancer is responsible for $90 \%$ of metastasis-related deaths and currently does not have a cure [1]. Statistically, the National Cancer Institute predicts five-year relative breast cancer patient survival is as low as $26 \%$ in patients detected with stage-IV breast cancer as opposed to $99 \%$ in patients detected with stage-I breast cancer (Figure 1-1) [2]. Therefore, research has been strife [3] in this arena of metastatic breast cancer to find novel avenues for therapeutic intervention in patients.

Gene expression profiling has shown that clinical outcomes of patients diagnosed with breast cancer can be predicted using a signature gene array expression pattern $[4,5]$. Specifically, breast tumors that occur by dysregulation of C-K-Ras proto-oncogene (KRAS) or HIF1A pathway show a higher proclivity for metastatic dissemination [6]. However, most researchers concede that metastasis proceeds in a sequential cascade of steps during which cells acquire specific characteristics for evading the immune system and enabling the cells to evade internal and environmental stressors[7]. Metastasis proceeds in a distinct cascade comprising the following sequence of steps:

- Epithelial-Mesenchymal Transition (EMT), migration and invasion: tumor cells become locally invasive and migratory and invade the basement membrane

- Intravasation: tumor cells enter the lymphatic and blood circulation

- Circulation: tumor cells get transported to distant tissues via lymph and blood flow

- Extravasation: tumor cells exit the blood circulation to distant secondary metastasis sites and may encounter dormancy

- Distant metastasis: tumor cells overcome hostile stress in the distant stroma, reinitiate growth and from micro-metastasis that eventually form macrometastasis.

\section{Prerequisites for Metastasis}

Many genes and signaling pathways converge to achieve each of the above-listed steps, endowing cancer cells with abilities to challenge the defined organization and established homeostasis of target organ, as shown in Figure 1-2 [8]. While metastatic sites may be pre-disposed as outlined in the famous "seed and soil" concept proposed by Stephan Paget [9], prerequisites for the initiation of the metastatic cascade are numerous and contain key elements such as: the intrinsic tumor-initiating capacity, altered cell adhesions, loss of cell adhesion, motility, resistance to extracellular death signals and 
A

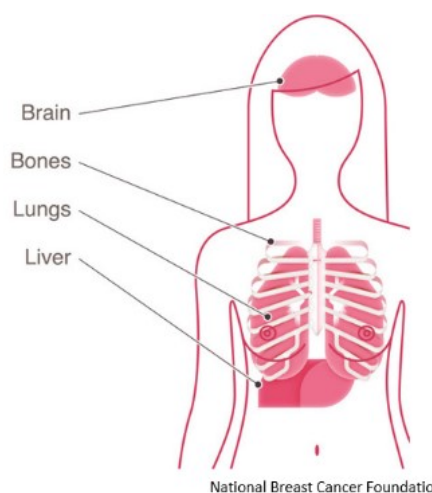

B

\begin{tabular}{|l|r|}
\hline $\begin{array}{l}\text { Estimated New } \\
\text { Cases in 2017 }\end{array}$ & 252,710 \\
$\begin{array}{l}\text { \% of All New } \\
\text { Cancer Cases }\end{array}$ & $15.0 \%$ \\
\hline \begin{tabular}{l|} 
Estimated \\
Deaths in 2017
\end{tabular} & 40,610 \\
\hline $\begin{array}{l}\text { \%of All } \\
\text { Cancer Deaths }\end{array}$ & $6.8 \%$ \\
\hline
\end{tabular}

C

Percent of Cases \& 5-Year Relative Survival by Stage at Diagnosis: Female Breast Cancer

Percent of Cases by Stage

5-Year Relative Survival

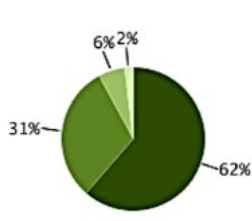

- Localized (62\%) Confined to
Primary Site

口 Regional (31\%) Spread to Regional
Lymph Nodes

Distant (6\%)

Metastasized

Unknown (2\%)
Unstaged

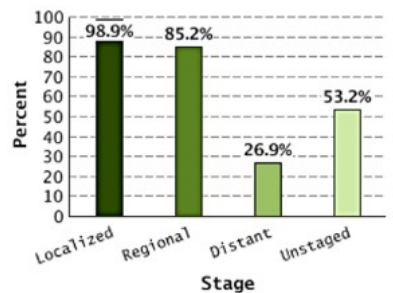

Stage

SEER 18 2007-2013, All Races, Females by SEER Summary Stage 2000

Cancer Statistics - National Cancer Institute

\section{Figure 1-1. Breast cancer metastasis.}

A. Breast cancer metastasis sites. Reprinted with permission from National Breast Cancer Foundation, Inc. (www.nationalbreastcancer.org/metastatic-breast-cancer) B. Breast cancer case statistics for year 2017. C. 5-year relative survival statistics for 2003-2013 Reprinted with permission from SEER Cancer Stat Facts: Female Breast Cancer, National Cancer Institute, Bethesda, MD.

(http://seer.cancer.gov/ststfacts/html/breast.html) 


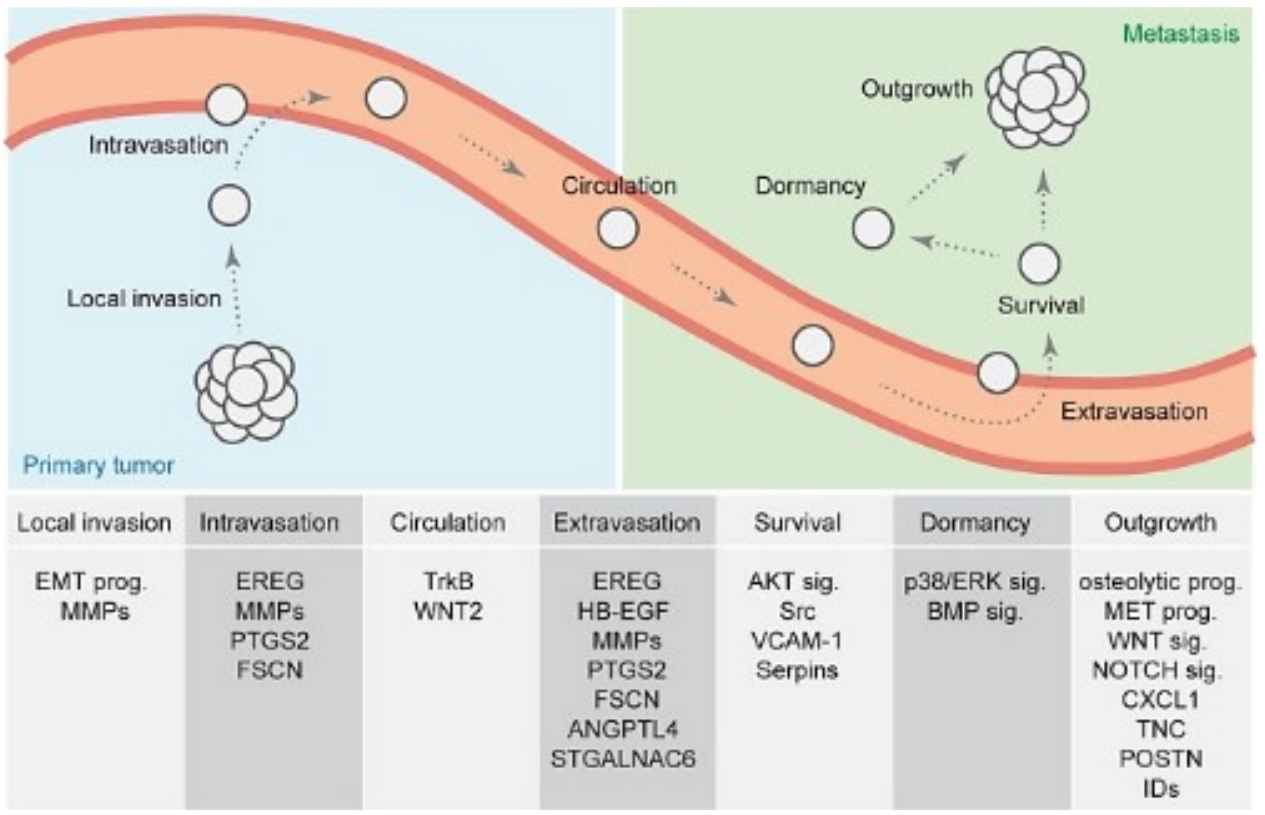

Figure 1-2. Metastatic cascade and signaling pathways involved.

Reprinted with permission from Xin Jin, Targeting Breast Cancer Metastasis. Breast Cancer (Auckl), 2015; 9(Suppl 1): 23-34. 
eventual disruption of basement membrane[10]. Various intrinsic and extrinsic factors such as those listed in Figure 1-3 fueled by genomic and epigenetic instabilities are activated in each of the stages of the cascade enabling tumor cells to maximize metastatic potential [10]. Al-Hajj et al., first used fluorescence associated cell sorting experiments (FACS) to show that a CD $44^{+} / \mathrm{CD} 24^{-/ 10 w}$ isolated cell population were more tumorigenic than other cell populations obtained from primary human xenograft tumors [11]. Since that integral discovery, many researchers have focused on the identification of markers linked with tumor initiation capacity. The evidence of self-renewal of a small subset of tumor cells is minimal and yet to fully elucidated. However, this property is considered an essential pre-requisite to initiate metastasis [10]. Metastatic cells also lose intercellular adhesiveness molecularly explained by the loss of $\mathrm{CDH} 1$ (E-cadherin) and gain of $\mathrm{CDH} 2$ (N-cadherin), leading to EMT. Following the loss of adhesion, metastatic cells gain motility via the upregulation of growth factor pathways. In MDA-MB-231, upregulation of epidermal growth factor receptors (EGFR) leads to increased invasiveness and cell motility, as shown in Matrigel-based invasion assays and scratch wound assays [12]. Evasion of apoptotic signals encountered by the metastatic cells which are generated by environmental stressors such as loss of adhesion, low nutrients, hypoxia and immune surveillance; is another vital prerequisite for metastatic cells [13]. Not only do metastatic cells evade immune surveillance but also manage to upregulate the secretion of a variety of matrix metalloproteases (MMP's), which enable cells to invade the basement membrane, a key process required to form an overt metastatic lesion [14].

Following intravasation, a whole new set of challenges and difficulty awaits a migratory cell. Hypoxia-activated matrix metalloproteases help in breaching the basement membrane as discussed above and cancer-associated fibroblasts have been implicated in the production of pro-metastatic cytokines like CXC motif chemokine ligand 12 (CXCL12), which facilitates intravasation, anoikis resistance and extravasation of cancer cells to distant organs $[15,16]$. The pattern of colonization can be defined by the seed and soil concept proposed by Paget. Once extravasation has occurred, cancer cells may enter a state of dormancy, but eventually the cell must overcome this state in order to generate overt metastatic lesions [17]. Several mechanisms are involved in surmounting this dormancy, which are specific to the secondary organ being colonized. For example, production of colony stimulating factor (CSF1), tumor necrosis factor ligand (TNFSF11), and interleukins (IL6, IL8, IL11) are essential for successful colonization of tumor cells in bones [10].

\section{Role of Tumor Hypoxia in Metastasis}

Tumor hypoxia is a dynamic condition that results in heterogeneity in tumor cell population and contributes to overall aggressiveness of the tumor [18]. Rapid utilization of oxygen and nutrients by the actively dividing tumor cells in solid tumors leads to the generation of hypoxia pockets and low nutrient availability, which is worsened by structurally and functionally abnormal vessels that form within solid tumors [19]. One way of measuring oxygen levels in tissue is by using electrodes to measure the partial 


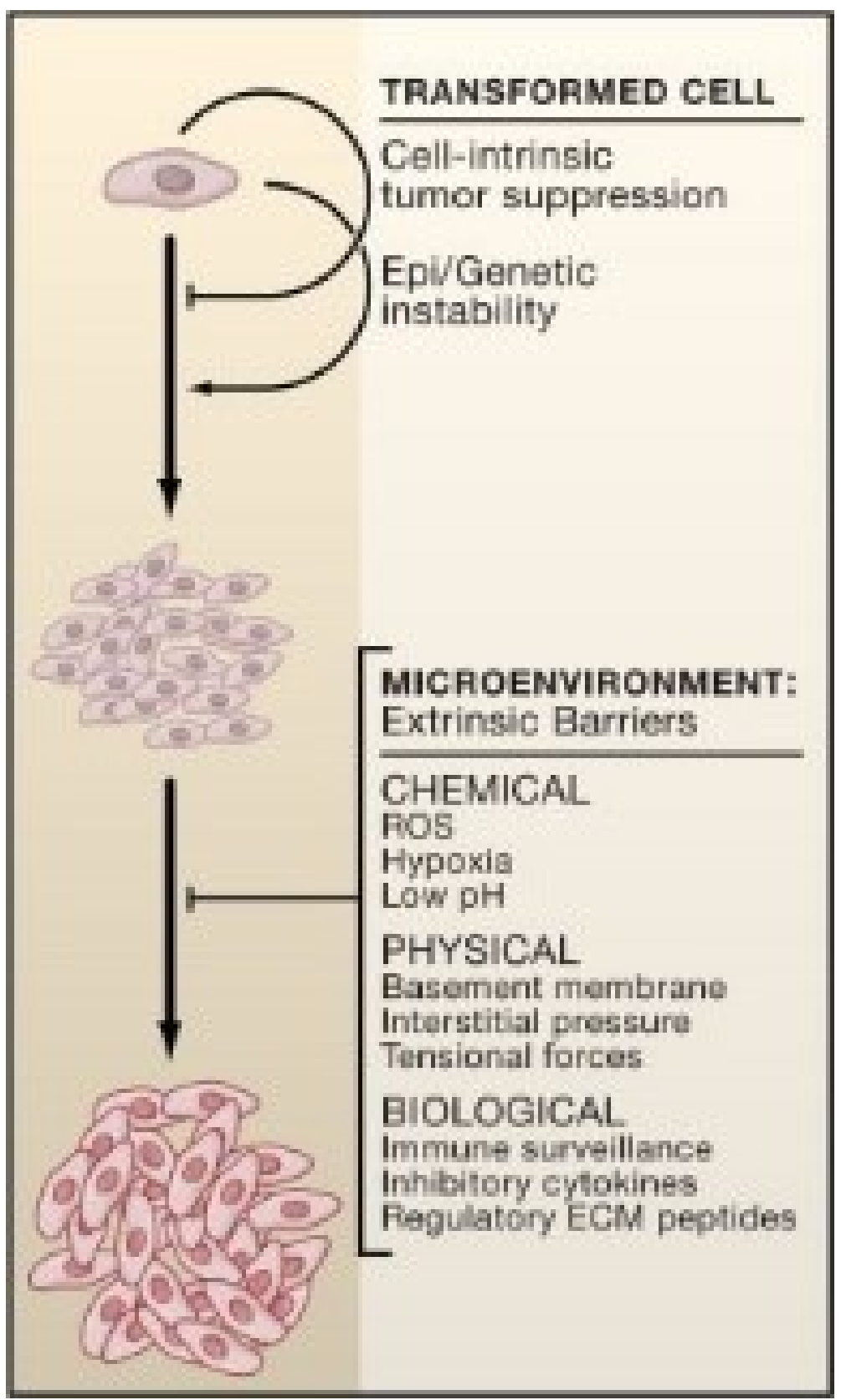

Figure 1-3. Cell intrinsic and extrinsic pressures that drive metastasis.

Reprinted with permission from Joan Massague, Cancer Metastasis: Building a Framework. Cell, Volume 127, Issue 4, p679-659, November 2006. 
pressure of oxygen $\left(\mathrm{pO}_{2}\right)$. The median $\mathrm{pO}_{2}$ in healthy human breast tissue is $65 \mathrm{~mm}$ of $\mathrm{Hg}$, and by contrast breast cancer tissues have a median $\mathrm{pO}_{2}$ of $10 \mathrm{~mm}$ of $\mathrm{Hg}$ [20]. $\mathrm{pO}_{2}$ of less than $10 \mathrm{~mm} \mathrm{Hg}$ has been linked to increased metastasis in breast cancer [21]. One explanation offered for this phenomenon is that intratumoral hypoxia acts as a pathological stimulus, driving changes in gene expression leading to aberrant gene expression patterns contributing to a metastatic phenotype. In primary tumor cells, for instance, hypoxia upregulates the expression of CXC motif chemokine receptor (CXCR4) which leads to an increase in the migratory and invasive phenotype of MDA-MB-231 and MCF-7 cells lines [22]. Tumor cells have been noted to employ multiple strategies to overcome the hypoxia stress, becoming remarkably tolerant to hypoxia stress [23]. However, prolonged hypoxia or nutrient deprivation leads to necrotic cell death, which are found in solid breast tumors. Hypoxia of primary tumors have also been shown to trigger systemic release of molecules such as angiopoietin like protein 4 (ANGPTL4) and lysyl oxidase (LOX) which prime the metastatic niches for subsequent colonization of metastatic breast cancer cells [24, 25].

Listed below are known mechanisms used by hypoxic stress to modulate metastatic potential:

- Cytokine secretion - HIF1A regulate secretion of various cytokines which help promote primary tumor cell migration, angiogenesis, and immune evasion. HIF1A can activate vascular endothelial growth factor (VEGF) production to promote angiogenesis, and induces release of $\mathrm{C}-\mathrm{C}$ motif chemokine ligand 5 (CCL5) and CXCL12 to recruit myeloid cells which elicit an inflammatory response, and promote resistance to T-cell mediated death by increasing expression of programmed death-ligand-1 (CD274) on tumor cells [26].

- Extracellular remodeling - Hypoxia can modulate the collagen present in the extracellular matrix via modulation of LOX family of proteins, they also upregulate secretion of matrix metalloproteinases that enable degradation of extracellular matrix (ECM) and aid migration of primary tumor cells [27].

- Regulation of immune cell infiltration - HIF1A deficient mice show delays in myeloid cell infiltrations proving the importance of hypoxia in macrophage recruitment in tumors. These macrophages secrete growth factors and cytokines such as VEGF, platelet-derived growth factor (PDGF), interleukins, and transforming growth factor beta (TGFB) which may attract further macrophages, activate stromal cells and aid in the survival and proliferation of primary tumor cells $[28,29]$.

- Metabolic adaptation - HIF1A mediated upregulation of glycolytic enzymes has been reported in several studies [30]. In order to maintain a high glycolytic rate, pyruvate is metabolized rapidly to lactate by HIF1A mediated upregulation of lactate dehydrogenate enzyme (LDHA) [31]. In conjunction with amplification of MYC proto-oncogene, HIF1A can also exploit glutamine metabolism which is also associated with increased aggressiveness of tumor cells [32]. HIF1A has also 
been shown to engage tumor protein 53 (TP53) mediated gene expression in tumor cells experiencing chronic or prolonged hypoxic stress to overcome reactive oxygen species (ROS) stress [33,34].

- Anoikis resistance - Loss of attachment to basement membrane can cause loss of survival signals in tumor cells [35]. HIF1A has been shown to upregulate ANGPTL4 and EGFR pathway, while downregulating BCL2 interacting mediator of cell death (BCL2L11/BIM), BCL2 modifying factor (BMF), and integrin alpha subunit - ITGA5, leading to evasion of anoikis (apoptotic pathway initiated in detachment cells) [36-39].

- Phenotypic plasticity (EMT/MET) - Rapid phenotypic change from epithelial to mesenchymal in the primary tumor site of the metastatic niche, plays a key role in metastasis [40]. Hypoxia has been known to promote EMT through direct HIF1A mediated transcriptional activation of Twist family basic helix-loop-helix transcription factor (TWIST) and zinc finger E-box binding protein (ZEB2), and also suppression of $\mathrm{CDH1}$ [41].

Not only is hypoxia implicated in the increase of metastatic potential of breast cancer cells but this state has also been linked to chemo- and radio-resistance. This observation dates to 1953 when Grey et al., showed that sensitivity of tumor cells to Xrays is about three times stronger when irradiated in an oxygenated medium as opposed to an anoxic medium [42]. Taken together these evidences suggest that hypoxia is an important multifaceted driver of metastasis, which leads to study of regulators in hypoxia pathway discussed below.

\section{Hypoxia Inducible Factor HIF1A Drives Metastasis}

Hypoxia is sensed by molecular dioxygenases such as prolyl dehydrogenases (PHD) in cells [43]. These oxidoreductase enzymes modify prolines in the oxygen-labile subunits of HIF in the presence of oxygen leading to an E3 dependent ubiquitination and degradation of HIF under average oxygen concentration [44]. In hypoxia, due to an absence of oxygen, PHD's are inactive, leading to an increase in HIF activity [45]. HIF can, directly and indirectly, regulate cellular processes like cell cycle, cell growth, migration, invasion, autophagy, chemo-resistance, and anoikis-resistance, all of which may increase the metastatic potential of cancer cells [46]. HIF also regulates chromatin remodeling through epigenetic modifications, post-translational histone modifications and DNA methyltransferases [47].

HIF are heterodimers comprising an oxygen-dependent subunit - HIF1-alpha (HIF1A/ HIF $1 \alpha$ ) and a constitutively expressed HIF 1-beta (HIF1B/ HIF $1 \beta)$ subunits. It is the HIF1A subunit that contains the oxygen-mediated PHD modifiable proline residues. Under hypoxia, HIF1A is stabilized, and over 70 downstream direct transcriptional targets and approximately 1500 indirect downstream HIF targets have been identified till 
date, all of which can help through the metastatic cascade of tumor cells [48] as shown in Figure 1-4.

HIF1A promotes primary tumor growth and vascularization via direct upregulation of VEGF and CXCL12. sh-RNA mediated knockdown of HIF1A alone, may reduce lymph node metastasis, and osteolytic lesions. Conditional knockout of HIF1A in epithelial cells in MMTV-PyMT mice has been shown to decrease spontaneous lung metastases by $\sim 50 \%$ [49]. During the metastatic cascade, HIF1A promotes EMT, migration, and invasion through the basement membrane by downregulation of CDH1 and upregulation of SNAI1 ( Snail family Zinc finger transcription factor), SNAI2, TWIST, TCF3 (Transcription factor 3), HGF (Hepatocyte growth factor), ITGA5, ITGA6, MMP2 and MMP9 [50]. Intravasation and extravasation of tumor cells occur via loosened junctions amongst epithelial cells facilitated by the abnormal structure and function of tumor-associated blood vessels. VEGFA, VEGFC and (PDGFB), which are well documented angiogenic factors, are directly upregulated by HIF1A and help in intratumoral vascularization, resulting in poorly constructed vasculature to facilitate intravasation or extravasation of tumor cells [51]. In addition, HIF1A mediated secretion of CXCL12 and CCL21(C-C motif chemokine ligand 21) that induce intravasation in breast cancer cells. Survival in lymphatic or blood circulation requires survival of breast cancer cells from anoikis, a form of cell death activated by loss of attachment. HIF1 has been shown to be activated upon loss of attachment and implicated in anoikis resistance via upregulating the EGFR- MAPK (mitogen activated protein kinase) pro-survival pathway [52]. Another target of HIF1A, L1 cell adhesion molecule (L1CAM) is a transmembrane protein which favorably mediates breast cancer cell and epithelial cell interactions aiding metastatic cells until they reach favorable metastatic niches to extravasate [50]. Specific cytokines have been shown to impart metastatic tropism to breast cancer cells. For example, HIF1A mediated expression of ANGPTL4 mediates tropism to lungs and CXCR4 expression confer tropism to bone [53, 54]. Upregulation of LOX and MMP's favorably allow for the formation of overt metastases [55]. It is proposed that metastatic cells revert back to epithelial phenotype via PHD-mediated inactivation of HIF in non-hypoxic conditions thus relieving repression of CDH1 [56]. These studies have led to the identification of a variety of drugs that inhibit HIF1 activity in mice as shown in Figure 1-5 [57].

Activation of HIF1A targets mentioned above could be by direct HIF1A-mediated transcriptional activation or indirectly via transcriptional factors induced by HIF1A. To bring about this activation, HIF can recruit chromatin modifying complexes such as SWI/SNF [58], ISWI [59] and several co-factors like CREB binding protein (CREBBP) and E1 binding protein 300 (EP300) [48]. Further study shows that regulation of downstream targets by HIF need a multiprotein complex which includes HIF1A, and the accessory transcription factor, p300/CREBBP [60,61]. Sequence analysis of regions surrounding the functional hypoxia-responsive elements reveal motifs for binding of other transcription factors such as FOS (Fos proto-oncogene), CREB (cAMP responsive element binding protein), NYF (nuclear transcription factor), and E2F (Eukaryotic transcription factor), suggesting a functional interplay amongst DNA binding proteins, that may modify the hypoxic response [60]. However, this co-factor recruitment and 


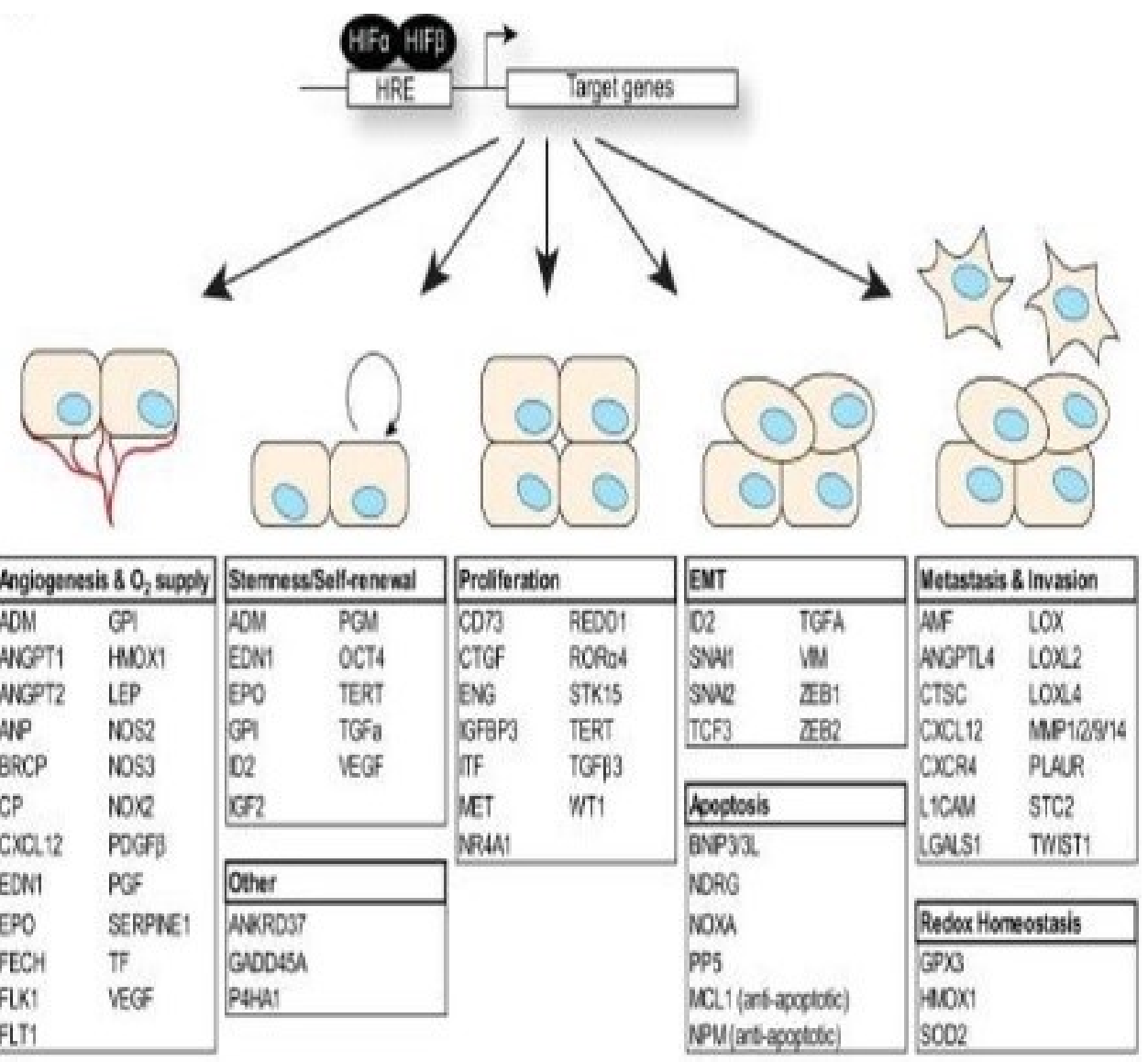

Figure 1-4. Transcriptional targets of HIF.

Seventy direct targets of HIF and the roles they orchestrate in the metastatic cascade. Reprinted with permission from Joaquin M Espinosa, Transcriptional Regulation by Hypoxia Inducible Factors. Crit Rev Biochem Mol Bio (HHS Author Manuscripts). 2014 Jan-Feb; 49(1): 1-15. 


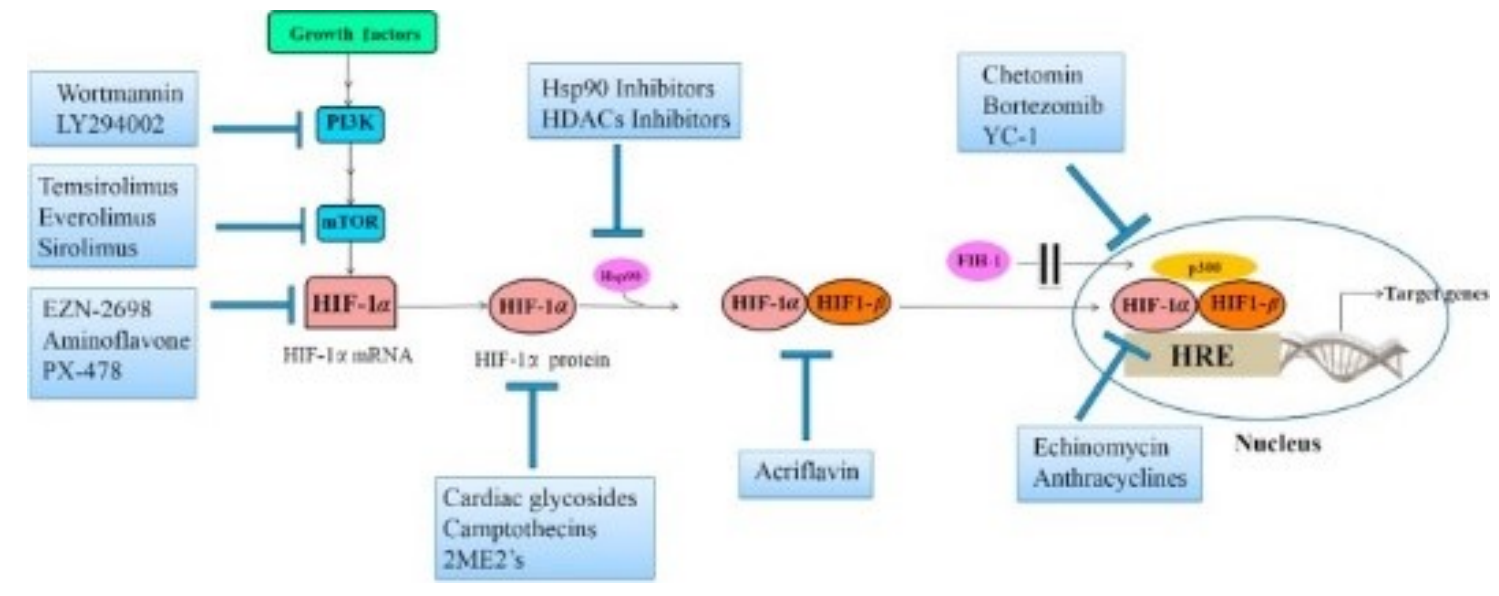

Figure 1-5. HIF1A inhibitors modulate different aspects of the HIF1A activation pathway.

Reprinted with permission fromWei Li, HIF-1 $\alpha$ pathway: role, regulation and intervention for cancer therapy.Acta Pharm Sin B (Elsevier), 2015 Sep, 5(5): 378-389. 
downstream pathway activation are cell context specific. Not only does the hypoxia response differ in different cell lines, a comparative study found the induction of HIF targets genes were higher in epithelial state compared to a mesenchymal state of the same cell line [48]. To compound its functional complexity, HIF1A may activate secondary downstream transcription factors like basic helix-loop-helix protein DEC1/ BHLHE40 that help to further orchestrate the hypoxic response [62]. HIF1A, their direct targets, transcriptional co-regulators, and co-factors, can all contribute to every step of the metastatic cascade discussed above and impart resistance to immune attack via several HIF-dependent mechanisms during the metastatic process $[29,50]$. Since the hypoxic response is one of the important drivers of metastasis, identifying the transcriptional coregulators of HIF1A may open doors to novel therapeutic interventions in patients.

\section{Screening for Transcriptional Regulators Involved in Metastasis of Breast Cancer Cells}

Transcription factors are favorably poised to rapidly activate or inactivate gene expression based on adjacent tumor cells and environmental cues, as shown in Figure 1-6 [63]. Therefore, it comes as no surprise, that transcription factors are often deregulated by tumor cells to aid with tumor progression and metastasis. Transcription factors can upregulate or downregulate expression of specific downstream genes upon receiving signals from 19 key signal transduction pathways [63]. As discussed previously, development of cancer requires a multiplex reversible phenotype and several nonMendelian epigenetic alterations that may account for mosaic or tissue-specific gene expression. These features make transcription factors attractive targets for cancer therapy. Many researchers have now focused their efforts on studying how transcription factors affect genetic and epigenetic gene expression which aids tumor cell survival and proliferation, uncovering novel therapeutic targets for a more targeted cancer therapy [64].

Many environmental cues including hypoxia have been shown to cause epigenetic modifications in solid tumors. The master hypoxia regulator HIF1A can cause both gene activation and repression imparting pro-survival ability, anti-apoptotic mechanisms, and resistance to radiation and chemotherapy in tumor cells, resulting in poor outcome in patients [65]. Several of these targets such as SNAI1 and TWIST are activated directly by HIF1A [50], but there are also a multitude of downstream targets whose activation is facilitated by other downstream transcription factors, epigenetic and chromatin remodelers, all of which are directly or indirectly influenced by HIF1A [48]. Many such downstream transcription factors and epigenetic modifiers such as histone methylases, demethylases, acetylases and chromatin remodelers, have been linked to an aggressive and malignant phenotype in patients. In this study, we dissect the role of such transcriptional modulators in metastasis of breast cancer.

BHLHE40/DEC1 is a basic helix loop helix protein reported to be a transcriptional repressor involved in the regulation of genes associated with circadian rhythm, apoptosis and differentiation [66]. Immunohistochemical studies show that 


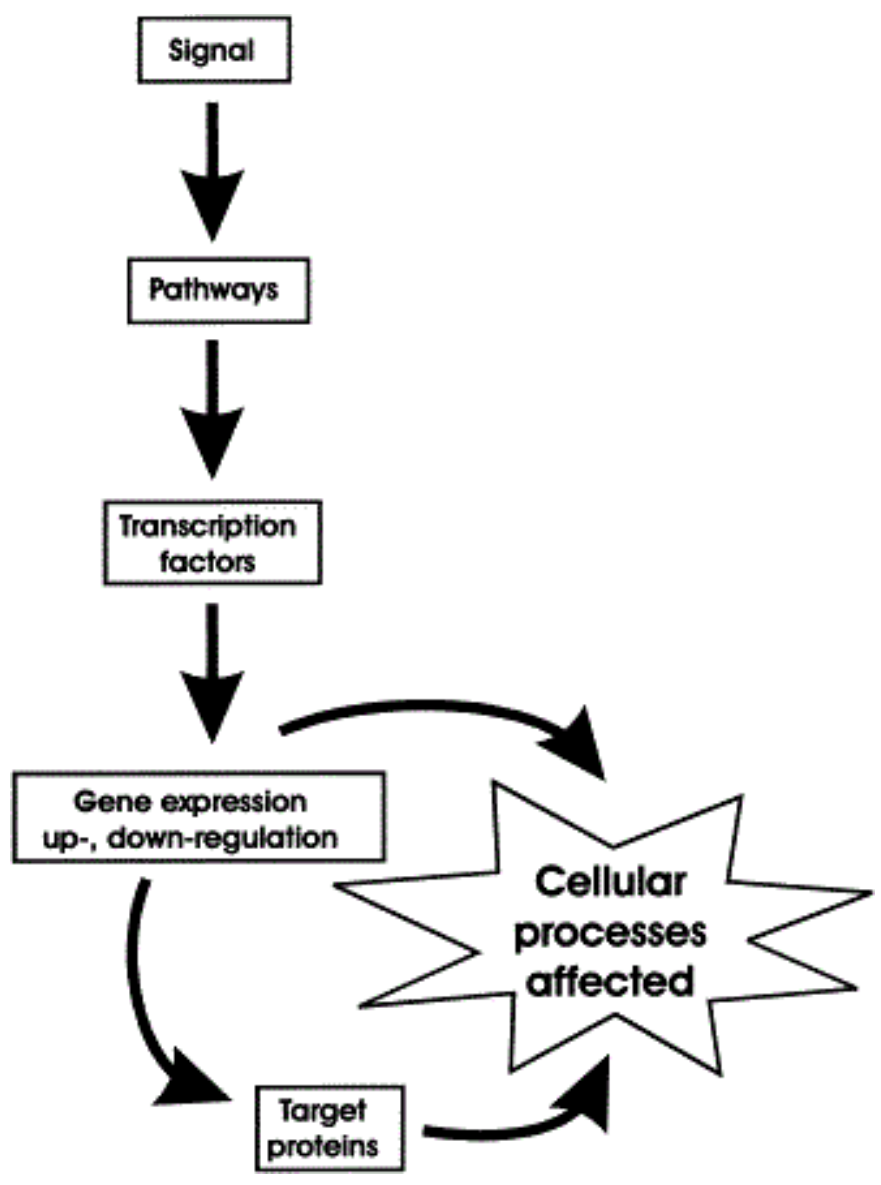

Figure 1-6. Transcription factor activity.

Simple flow diagram of transcriptional factor mediated pathway activation. Reprinted with permission from Daniel W Nerbert, Transcription factors and cancer: an overview. Toxicology (Elsevier), Volumes 181-182, 27 December 2002, Pages 131-141. 
BHLHE40 is associated with tumor grade in patient-derived breast cancer tissues [67]. Overexpression of BHLHE40 can repress cell proliferation under normal conditions, but in a hostile environment like hypoxia or low nutrition, BHLHE40 upregulation can inhibit cell apoptosis and increase tumorigenicity [68]. Under hypoxic stress, BHLHE40, a direct target of HIF1A [36] is rapidly upregulated and has been shown to increase the invasiveness of breast cancer cell lines and impart metastatic potential [69]. BHLHE40 has also been shown to increase breast cancer cell proliferation by increasing cyclin $\mathrm{E}$ levels [70]. BHLHE40 mediated suppression of clock (CLOCK/ BMAL1/2) genes activation, in turn, has been implicated in the modulation of drug toxicity [68]. Therefore, findings to date show that BHLHE40 plays an important role in the cancer progression and modulation of tumor response to chemotherapy [71]. However, how BHLHE40 modulates these responses or tumor progression is unclear.

HIF1A stimulates the expression of KDM6B [72], a lysine-specific histone demethylase that demethylates di- or tri- methylated lysine 27 of histone H3, causing gene expression. Since it is directly poised to activate genes in answer to up-stream cues, KDM6B has been investigated for its expression in various tumors. Specifically, KDM6B is known to increase invasiveness and promote epithelial-mesenchymal transition of breast cancer cells MDA-MB-231 cells via transcriptional upregulation of SNAI1 expression [73]. EHMT2, another histone modifier modulated by hypoxia [74], is a histone lysine methyltransferase localized in euchromatin, which acts as a co-repressor along with partner transcription factors [75]. Increased expression of EHMT2 is observed in clinical cancer tissues and knockdown of EHMT2 in-vitro induces apoptotic cell death in various cancer cell lines [76].

Finally, studies have focused intently on chromatin remodelers to elucidate their roles in hypoxia response modulation, cancer development and disease progression. Chromatin may assume a state as either euchromatin, containing loosely bound actively transcribed genes; or as heterochromatin, consisting of compact chromatin associated with silent genes [77]. Changes in chromatin structure is required for most cellular processes. Cells, therefore, have strategies in place for relaxing or tightening chromatin based on cellular cues or requirements. One such mechanism is using ATP-mediated chromatin remodelers. The well-characterized SWI/SNF (Switch/Sucrose Nonfermentable Complex) is a chromatin remodeler involved in hypoxia response [58]. $\mathrm{SWI} / \mathrm{SNF}$ complex is evolutionarily conserved multi-subunit complex, containing the catalytic subunits with ATPase activity (SMARCA2/BRM or SMARCA4/BRG1) and other accessory subunits. Presence of SMARCA4 and SMARCA2 at the EPO promoter under hypoxia demonstrated the importance of this complex in HIF1A mediated activation of EPO gene[78]. SMARCA4 deletion also led to defects in erythropoiesis and vascular development in mouse models, which overlap with the known function of HIF1A targets such as VEGF. Mechanistically, HIF1 and HIF2 recruit SMARCA4 containing SWI/SNF complex to HIF target genes promoter to facilitate ATP dependent chromatin remodeling and activation of HIF target genes [79]. These studies reflect the importance of SWI/SNF remodelers in hypoxia response, but the role of the accessory subunits and their mechanistic link to HIF1A need further investigation. Since research is now focusing on the importance of chromatin remodelers in hypoxia-mediated 
metastasis, the SWI/SNF complex has received high levels of scrutiny and the functions of its subunits are being individually studied to identify new avenues to target metastatic disease progression.

In this study, we analyzed two subunits of the SWI/SNF complex, SMARCE1 and SMARCA4, for their roles in breast cancer metastasis. Genome-wide transcriptome studies revealed that BRG1 controls the expression of PI3K/ AKT and BCL2 which are frequently altered in tumor cells [80]. In breast cancers, SMARCA4 is involved in progression and metastasis, therefore may serve as a novel biomarker, predictive of distant metastasis and patient outcomes [81]. SMARCE1, however does not possess any helicase or ATPase activity but has still been associated with invasive progression of breast cancer [82]. SMARCE1/BAF57 is an accessory subunit of the SWI/SNF complex whose function is not completely unraveled.

Orthotopic xenograft models were used to deduce the role of these transcriptional modulators in MDA-MB-231 cells, a triple negative breast cancer cell-line. A lung metastatic derivative of MDA-MB-231 (LM) established as previously described [83] was used to engineer derivatives stably expressing short hairpin RNA (shRNA) against KDM6B, EHMT2, SMARCA4, SMARCE1, BHLHE40 or empty vector (EV) through lentiviral transduction and selected in medium containing respective selection drug as shown in Table 1-1. Mice inoculated with tumor cells were inspected weekly for tumor appearance by visual observation and palpation. Primary tumor outgrowth was quantified twice a week using digital calipers. Tumor volume was calculated as: Volume $=\left(\right.$ width $^{2} \mathrm{x}$ length) $/ 2$. Tumor and lung tissues were extracted 6 weeks after inoculation. The left lung lobes were imaged using fluorescent microscopy, and metastatic foci in lungs were quantified using ImageJ software (Figure 1-7).

\section{BHLHE40 and SMARCE1 Knockdown Reduces Lung Metastasis of Breast Cancer In-Vivo}

Primary tumor growth rate was significantly reduced in the BHLHE40 knockdown cells as shown in Figure 1-8A. Spontaneous lung metastatic foci were measured in percentage metastatic foci occupied in the lung parenchyma following six weeks of cell inoculation in the mammary fat pad of NSG mice. The mean metastatic foci of the LM cells and derivatives are as shown in Table 1-2. BHLHE40-KD (knockdown) and SMARCE1-KD both showed a substantial decrease in lung metastatic foci in lungs of mice, as shown in Figure 1-8B and C. In addition, BHLHE40 was shown to be essential for primary tumor growth. This study further investigates the underlying mechanisms which lead to reduction in the metastatic potential of breast cancer cells by BHLHE40-KD and SMARCE1-KD. 
Table 1-1. shRNA construct information.

\begin{tabular}{|c|c|c|c|}
\hline Gene name & Catalog\# & Company & Selection drug \\
\hline \multirow{2}{*}{ SMARCA4 } & \multirow[t]{2}{*}{ pBABE BRG1 } & Addgene & $2 \mu \mathrm{g} / \mathrm{ml}$ Puromycin \\
\hline & & Cambridge, MS, USA & Sigma-Aldrich \\
\hline \multirow{2}{*}{ BHLHE40 } & \multirow{2}{*}{ TRCN0000232187 } & Sigma-Aldrich & $2 \mu \mathrm{g} / \mathrm{ml}$ Puromycin \\
\hline & & St.Louis, MO, USA & Sigma-Aldrich \\
\hline \multirow{2}{*}{ KDM6B } & \multirow{2}{*}{ MSCV JMJD3 } & Addgene & $2 \mu \mathrm{g} / \mathrm{ml}$ Puromycin \\
\hline & & Cambridge, MA, USA & Sigma-Aldrich \\
\hline \multirow{2}{*}{ SMARCE1 } & \multirow{2}{*}{ TRCN0000015779 } & Sigma-Aldrich & $2 \mu \mathrm{g} / \mathrm{ml}$ Puromycin \\
\hline & & St.Louis, MO, USA & Sigma-Aldrich \\
\hline \multirow{2}{*}{ EHMT2 } & \multirow{2}{*}{$\begin{array}{l}\text { pLenti6-MK1- } \\
\text { EHMT2-V5 }\end{array}$} & Addgene & $30 \mu \mathrm{g} / \mathrm{ml}$ Blasticidine \\
\hline & & Cambridge, MA, USA & Sigma-Aldrich \\
\hline \multirow{2}{*}{$\begin{array}{l}\text { Control- } \\
\text { mCherry }\end{array}$} & \multirow{2}{*}{ pEZX-AM03 } & GeneCopoeia & $2 \mu \mathrm{g} / \mathrm{ml}$ Puromycin \\
\hline & & Rockville, MD, USA & Sigma-Aldrich \\
\hline
\end{tabular}

Plasmids used for knockdown of gene of interest and the selection drug used for generation of stable knockdown cell line.

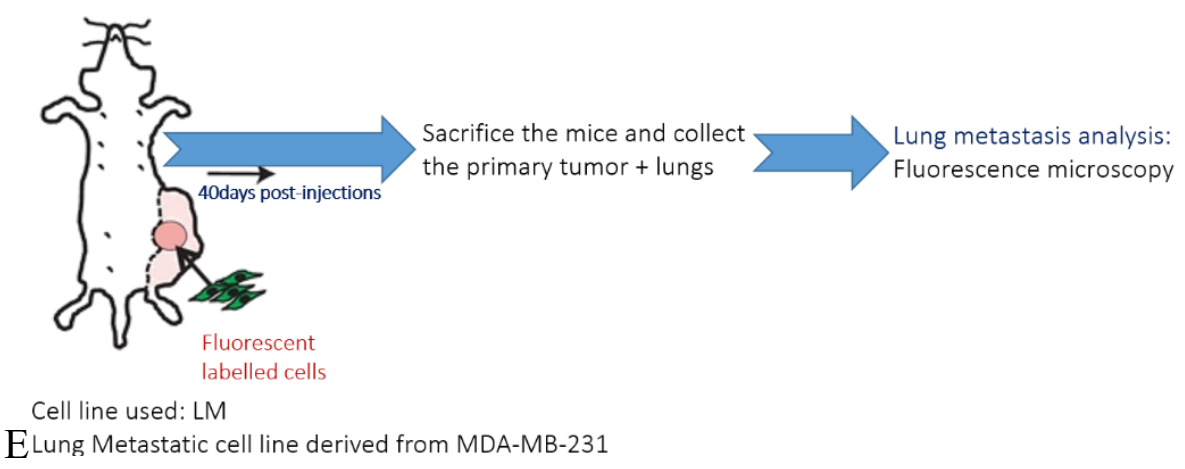

\section{Figure 1-7. Orthotropic Xenograft model studies.}

$5 \times 10^{5}$ fluorescently labelled cells were injected in the mammary fat pad of 4 weeks old NSG female mice and 40 days post injections the lungs were harvested and analyzed under the fluorescence microscope to detect the presence of metastatic foci. 
A

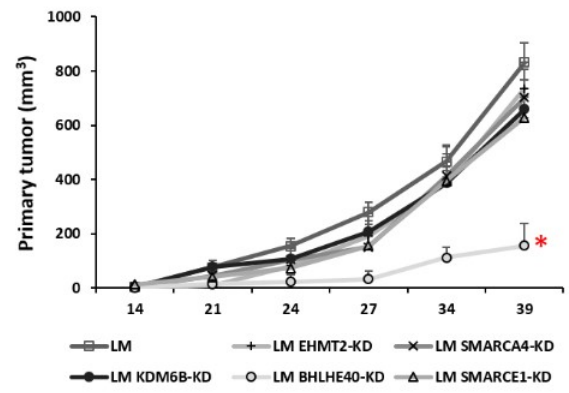

B

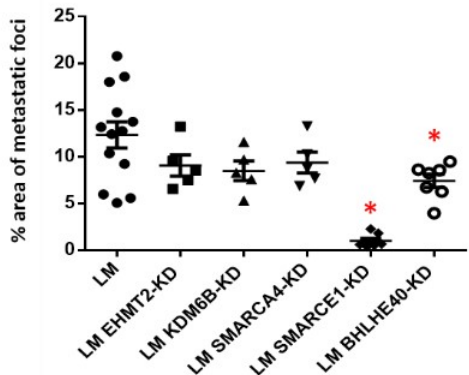

C

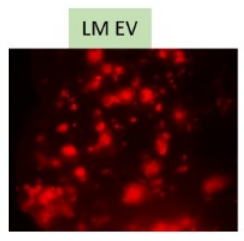

LM KDM6B-KD

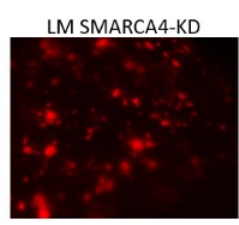

LM BHLHE40-KD
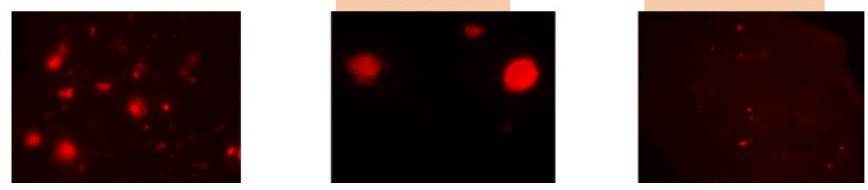

Figure 1-8. Fluorescent Lung metastases.

A. Primary tumor growth rate and B. percentage fluorescent lung metastatic foci in mice injected with LM cells transfected with empty vector (LM EV) and the knockdown derivatives, calculated using ImageJ software. $p<0.05, n=10$ C. Fluorescence pictures of lung metastasis pattern (10X).

Table 1-2. Mean percentage of metastatic foci in-vivo.

\begin{tabular}{lcc}
\hline Cell line & Mean \% lung metastatic foci & P value \\
\hline LM & $12.35 \pm 5.1$ & \\
LM EHMT2-KD & $9.08 \pm 2.6$ & 0.094 \\
LM KDM6B-KD & $8.51 \pm 2.3$ & 0.061 \\
LM SMARCA4-KD & $9.38 \pm 2.5$ & 0.115 \\
LM SMARCE1-KD & $1.02 \pm 0.7$ & $<0.00001$ \\
LM BHLHE40-KD & $7.42 \pm 1.9$ & 0.012 \\
\hline
\end{tabular}

Area of mean metastatic foci in mice lung parenchyma inoculated with LM cell lines and knockdown derivatives. (Highlighted cells show significant reduction in lung metastatic foci, $\mathrm{p}<0.05, \mathrm{n}=10$ ) 


\section{CHAPTER 2. ROLE OF SMARCE1 IN METASTATIC BREAST CANCER*}

\section{Introduction}

Breast cancer is the most frequently diagnosed form of cancer in American women and is the second leading cause of cancer related deaths, primarily due to the incurable nature of metastatic breast cancer [84]. Metastasis is the process whereby cancer cells spread from the site of the primary tumor to distant organs[10]. A stepwise sequence of events is involved in the metastatic process, during which the tumor cells encounter many environmental challenges such as loss of attachment to the extracellular matrix, physical stress during circulation in blood/lymph vessels and immune surveillance $[85,86]$. Only a small fraction of disseminated cells can alter gene expression patterns to obtain new phenotypic features according to environmental cues, and ultimately survive and adapt to form metastatic lesions. The mechanics of this adaptability is an evolving field of study.

Chromatin remodeling plays a key role in tumor progression by altering gene expression patterns to facilitate rapid adaptation of tumor cells to extracellular stimuli [87]. Aberrant chromatin remodeling activity is frequently detected in human tumors. High-throughput genome and/or exome sequencing studies have revealed that the SWI/SNF (switching-defective and sucrose non-fermenting) chromatin-remodeling complexes are the most highly mutated chromatin regulatory complexes, with nearly $20 \%$ of human cancers harboring mutations in at least one of the genes encoding SWI/SNF subunits [88]. Human SWI/SNF complexes consist of either BRG1/SMARCA4 or BRM/SMARCA2 subunit that possesses helicase and ATPase activity, and several BRG/BRM associated factors (BAFs) that regulate the DNA binding specificity and affinity of the complexes $[88,89]$. The cancer-associated mutations cause aberrant activation of the residual SWI/SNF complexes due to distinct subunit configurations, rather than eliminating the chromatin remodeling activity [90-92]. Although earlier studies have identified several SWI/SNF subunits (e.g., SMARCB1 and SMARCA4) as tumor repressors, emerging evidence suggests that SWI/SNF activity is essential for tumor initiation, maintenance and progression $[92,93]$. Therefore, it becomes critically important to define the role of each SWI/SNF subunit in development and progression of various types of tumors and adaptability of tumor cells to environmental cues.

Genomic amplification of BAF57/SMARCE1, a core subunit of human SWI/SNF complexes that play a role in interaction with transcription factors and chromatin, has been linked to a high-risk of recurrence of estrogen/progesterone receptor-negative breast tumors [94]. However, the underlying molecular mechanisms remain undefined. In this study, we demonstrated that SMARCE1 plays a key role in breast cancer metastasis by protecting breast cancer cells during circulation against anoikis through the HIF1A/PTK2 pathway.

*Adapted with permission. Fan M, Sethuraman A, et al. "Role of SMARCE1 in metastatic breast cancer” Breast Cancer Res 2016 Aug 5;18(1):81. [95] 


\section{Methods}

\section{Cell culture}

All cell lines were purchased from ATCC (Manassas, VA). MDA-MB-231, HCC38, BT549 and their derivatives were maintained in Minimum Essential Media (Thermo Fisher Scientific) supplemented with 10\% fetal bovine serum (FBS), 200 units/ml penicillin-streptomycin (Cellgro, Manassas, VA, USA) and $0.5 \mu \mathrm{g} / \mathrm{ml}$ Amphotericin B (Cellgro, Manassas, VA, USA). 184B5 cells were cultured in Mammary Epithelial Cell Growth Medium (MEGM, Lonza, Switzerland). A lung metastatic derivative of MDA-MB-231 (LM) was established as previously described [83]. Cells stably expressing short hairpin RNA (shRNA) against SMARCE1, HIF1A, PTK2 or empty vector (EV) control were established by lentivirus transduction and selected in medium containing $2 \mu \mathrm{g} / \mathrm{ml}$ puromycin (Sigma, St Louis, MO, USA). pLKO.1HIF1AshRNA (NM_001530.x-1048s1c1), pLKO.1-SMARCE1shRNA (TRCN0000015779) and pLKO.1-PTK2shRNA (TRCN0000344599) were obtained from Open Biosystems/GE Dharmacon (Lafayette, CO, USA) and Sigma-Aldrich (St. Louis, MO, USA). Cells stably expressing FAK/PTK2 or SMARCE1 were obtained by lentiviral transduction and selected in medium containing $30 \mu \mathrm{g} / \mathrm{ml}$ blasticidine (Sigma). pLX304-PTK2 (HsCD00442503) and pLX304-SMARCE1 (HsCD00440078) were purchased from DNASU Plasmid Repository (Tempe, AZ). Control cells were established by lentivirus transduction to express selection marker only. Accell SMARTpool (a mixture of 4 siRNA provided as a single reagent) for human SMARCE1 was obtained from Dharmacon (GE Healthcare Life Sciences, PA) and used to knockdown SMARCE1 expression by following the manufacture's protocol. The Accell Human Control siRNA (non-targeting) was used as control oligonucleotides.

\section{Orthotopic xenograft model and experimental lung metastasis model (tail-vein injection)}

All animal studies adhered to protocols approved by the Institutional Animal Care and Use Committee of University of Tennessee Health Science Center. For orthotopic xenograft model in NSG mice (NOD.Cg Prkdcscid Il2rgtm1Wj1/SzJ, The Jackson Laboratory), cells $\left(7.5 \times 10^{5}\right.$ cells in $10 \mu$ l Phosphate-buffered saline) were surgically inoculated into the right inguinal mammary gland fat pads of 4-week old female mice as previously described [96]. Mice were inspected weekly for tumor appearance by visual observation and palpation. Primary tumor outgrowth was monitored twice a week using digital calipers. Tumor volume was calculated as: Volume $=\left(\right.$ width $^{2} \mathrm{x}$ length $) / 2$. Tumor and lung tissues were extracted 6 weeks after inoculation. The left lung lobes were imaged under the fluorescent microscope, fixed with 4\% paraformaldehyde, followed by tissue section $(10 \mu \mathrm{m})$ and hematoxylin and eosin staining. Metastatic foci in lungs were quantified using ImageJ software. For experimental lung metastasis model, cells were inoculated into to 4-week old NSG mice by tail vein injection. Whole blood was collected by cardiac puncture at various time and subjected to ficoll-paque separation as 
per manufacturer's protocol immediately after collection (Ficoll-Paque PLUS, GE Healthcare Life Sciences, Piscataway, NJ) to isolate circulating tumor cells. Fluorescent circulating tumor cells were counted under fluorescent microscope using a 10X objective.

\section{Quantitation of mRNA using qPCR}

Total RNA was prepared with Trizol (Life technologies, Grand Island, NY), chloroform and isopropanol. mRNA concentration and quality was analyzed on the NanoDrop (ThermoScientific, USA). cDNAs for mRNA were synthesized by using iScript cDNA Synthesis Kits (BioRad, Hercules, CA). qPCR was performed on the CFX96 ${ }^{\mathrm{TM}}$ Real-Time PCR Detection System using SYBR Green Supermix (BioRad). Expression data of mRNA were normalized by the $2-\Delta \Delta \mathrm{CT}$ method to RPL13A, and presented as mean $\pm \mathrm{SE}(\mathrm{n}=3)$. qPCR primers were obtained from PrimerBank [97].

\section{Migration and invasion assays}

Cells (20,000 cells $/ 0.5 \mathrm{ml} /$ well) were plated onto control membrane inserts with 8 -micron pores (migration assay) or Matrigel-coated membrane transwell inserts (invasive assay) (BD Biosciences, Bedford, MA), which were placed in 24-well chambers filled with $0.6 \mathrm{ml}$ growth medium. Twenty-four hours after plating, cells that remained on the upper surface of the membrane were removed by cotton tipped swabs, and cells that migrated/invaded to the lower surface of the membrane were viewed under the fluorescent microscope. The fluorescent cell pictures were analyzed on ImageJ and percent migration was expressed as $\%$ migration $=$ (mean number of cells migrating through Matrigel insert membrane x 100/ mean seeding control) and percent invasion was expressed as: \% invasion $=$ (mean number of cells invading through Matrigel insert membrane $\mathrm{x} 100$ ) / mean number of cells migrating through control insert membrane.

\section{Suspension culture and inhibitor assays}

To mimic loss of attachment, $10^{5}$ cells/well were seeded in 6-well culture dishes coated with polyHEMA (Sigma, St Louis, MO, USA) to prevent cell adherence for various periods of time. Cells were inoculated with $3 \mathrm{ml}$ of growth medium initially with no further manipulation throughout the course of the experiment. Cell suspensions were placed into standard culture dishes 16 hours prior to end-point viable cell counting.

To identify the signaling pathways that promote survival of detached cells, a panel of chemical inhibitors were added in culture medium to cells plated in polyHEMA coated dishes: PTK2/FAK inhibitor - $0.5 \mu \mathrm{M}$ PF562271 (Selleckchem, Houston, TX), MAPK1/2 inhibitor $-10 \mu \mathrm{M}$ U0126 (LC Laboratories, Woburn, MA) or $10 \mu \mathrm{M}$ PD98059 (Santa Cruz Biotechnology, Dallas, TX), AKT inhibitor - $30 \mu \mathrm{M}$ ZSTK474 (LC Laboratories), TGFBRI/II inhibitor - $10 \mu \mathrm{M}$ LY2109761 (Selleckchem), SRC inhibitor $-3 \mu \mathrm{M}$ Dasnitib (LC Laoratories) or Notch inhibitor $-5 \mu \mathrm{M}$ DAPT 
(Selleckchem). Following a 7-day incubation, cells were plated back on the standard culture dishes and quantified 16 hours later.

\section{Protein extraction and immunoblotting}

Protein extraction from whole cells was prepared with radioimmunoprecipitation buffer, cytosolic and nuclear fractions was prepared by NE-PER Nuclear and Cytoplasmic Extraction Reagents (ThermoScientific). Protein lysates were resolved on 8$10 \%$ SDS-PAGE and transferred to polyvinylidene fluoride membrane and immunoblotted with the following antibodies: anti-PTK2/FAK, FAK-Tyr576P/Tyr577P, MAPK1/2-Thr202P/Tyr204P, MAPK1/2, AKT-Tyr416P, AKT and BCL2L11 from Cell Signaling Technologies (Boston, MA); anti-HIF1A from Novus Biologicals (Littleton, CO); anti-SMARCE1 and SMARCA4 from Bethyl Laboratories (Montgomery, TX); anti-GAPDH from Millipore (Merck, Darmstadt, Germany) and anti-TBP from Abcam (Cambridge, MA). Luminescence detection of these membranes were performed as previously discussed [83].

\section{Chromatin immunoprecipitation}

Cells $\left(2 \times 10^{7}\right)$ were cross-linked with $1 \%$ formaldehyde (Pierce ${ }^{\mathrm{TM}} 16 \%$ Formaldehyde (w/v), Methanol-free, Life Technologies) for 15 minutes. The nuclear fraction was prepared using the NE-PER ${ }^{\mathrm{TM}}$ Nuclear and Cytoplasmic Extraction Reagents (Life Technologies), chromatin was sheared by sonication to obtain $500 \mathrm{bp}$ fragments and the soluble fraction was collected after centrifuge (12,000rpm, $15 \mathrm{~min})$. The soluble nuclear fractions were pre-cleaned with control IgG and MagnaBind Goat anti-Rabbit IgG (Life Technologies) and used for immunoprecipitation with antibodies against HIF1A, SMARCE1 or SMARCA4. Immunocomplexes were isolated with MagnaBind Goat anti-Rabbit IgG, de-crosslinked, and subjected to DNA preparation using the MiniElute PCR Purification Kit (QIAGEN, Germantown, MD). qPCR was performed to detect the presence of PTK2 promoter region by using primers (Forward: 5'-CTCTTCCTCCTCCTGCCTCT-3'; reverse 5'-GTTCGGGGAAGACAGAAA GG$\left.3^{\prime}\right)$. To detect protein-protein interaction, the immunocomplexes were dissolved in Laemmli Sample Buffer (Bio-Rad) and subjected to immunoblotting assay.

\section{Caspase assay}

$5 \times 10^{5}$ cells were plated in polyHEMA coated dishes and Caspase- 3 levels were measured at indicated time points using the Caspase-Glo ${ }^{\circledR}$ 3/7 assay kit (Promega). The cells were lysed with lysis buffer provided with the kit and substrate and lysis mix was incubated for 30 minutes with rotation and quantifies via luminometer. Caspase activity was calculated as per manufacturer protocol and, normalized to cell numbers. 


\section{Nucleosome scanning assay (NUSA)}

$10^{6}$ cells were placed under suspension culture in polyHEMA coated dishes for 0 , 0.5, 1 and 2 hours, respectively. Nuclei were isolated, treated with Atlantis Double-strand specific DNased (dsDNase) and subjected to DNA purification according to the EZ Nucleosomal DNA prep kit (Zymo Research). qPCR was performed to determine nucleosome positioning on the $P T K 2$ promoter. Overlapping primers were designed from -150 to +1589 relative to start site of $P T K 2$ promoter to generate amplicons of $\sim 150 \mathrm{bp}$, the size of DNA covered by one nucleosome. DNA amount was calculated according to a standard curve (qPCR CTs $v s$. various concentrations of template) generated for each primer and normalized to qPCR CTs of DNA purified from equal number of nuclei untreated with dsDNAase.

\section{Statistical analysis}

ANOVA and post-hoc least significant difference analysis or t-tests were performed using GraphPad Prism 5 software. P values $<0.05(*)$ were considered statistically significant. Data from two or three independent experiments with replicates are presented as means $\pm \mathrm{sd}$.

\section{Results}

\section{SMARCE1 knockdown reduces lung metastasis of breast cancer in vivo}

To define the role of SMARCE1 in breast cancer metastasis, we examined the effect of SMARCE1 knockdown on spontaneous lung metastasis using an orthotopic xenograft mouse model derived from a lung metastatic variant of MDA-MB-231 cells, previously described and designated as LM [83]. SMARCE1 knockdown showed no significant effect on the latency and growth rate of primary xenografts in mammary gland fat pads (Figure 2-1A and B, LM-SMARCE1-KD vs. LM-EV), but substantially reduced both the number and size of metastatic foci in lungs (Figure 2-1C, LM-SMARCE1-KD vs. LM-EV). According to the images of lung tissue sections, metastatic foci occupied $12.30 \pm 3.87 \%$ of the lung parenchyma in mice 6 -wk after inoculation with LM-EV cells, which was reduced to $1.02 \pm 0.76 \%$ in mice inoculated with LM-SMARCE1-KD cells $(p=0.0002)$ (Figure 2-1C, bar graph). By examining the number of tumor cells in blood, we found that SMARCE1 knockdown significantly reduced the number of circulating tumor cells ( $p=0.0011)$ (Figure 2-1D) in mice inoculated with LM cells. Together, these results suggest that SMARCE1 activity is dispensable for primary tumor outgrowth, but essential for distant metastasis of MDA-MB-231 cells. 
A
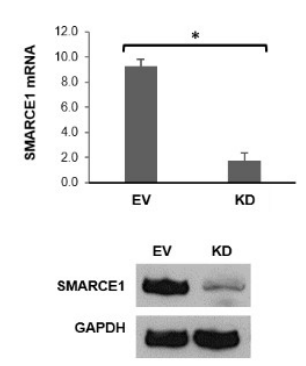

B

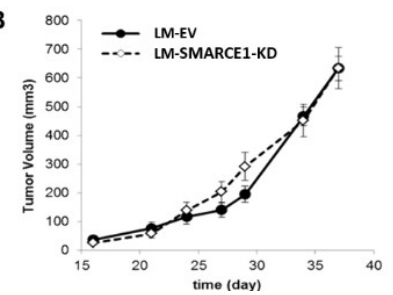

C

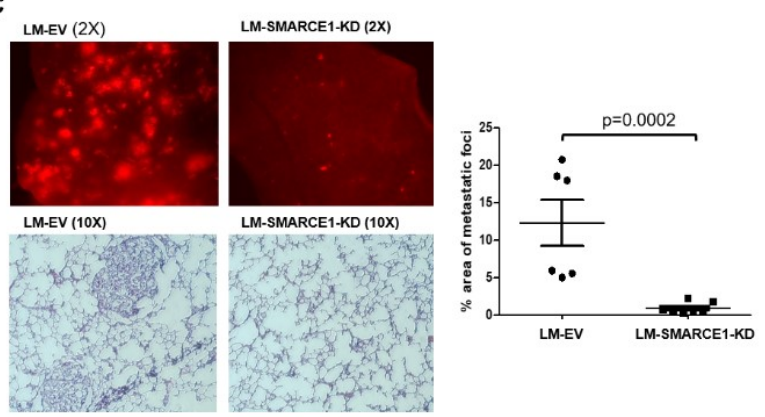

D

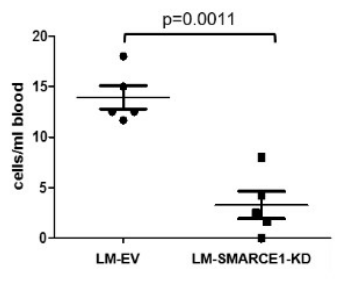

Figure 2-1. SMARCE1 knockdown reduces lung metastasis of breast cancer in vivo.

A. Expression levels of SMARCE1 mRNA and protein in LM-EV and LM-SMARCE1$\mathrm{KD}$ cells B. Effect of SMARCE1 knockdown on the growth of xenografts in the fourth inguinal mammary fat pads of female NSG mice, $n=6 \mathbf{C}$. Spontaneous lung metastasis from orthotopic sites. Metastatic foci of tumor cells expressing red fluorescent protein on the dorsal surface of the left lung lobe were imaged 38 days after tumor cell inoculation (left panel). The presence of tumor cells in the lungs was visualized by hematoxylin and eosin $(\mathrm{H} \& \mathrm{E})$ staining of formalin-fixed lung sections $(10 \mu \mathrm{M})$ and quantified by Image $\mathrm{J}$ software (right panel) $n=6$. D. Number of circulating tumor cells in blood. Fluorescent tumor cells in mouse blood were isolated and counted 38 days after tumor cell inoculation. $(\mathrm{n}=5)$ 


\section{SMARCE1 knockdown reduces lung colonization of tumor cells inoculated through tail vein}

Metastasis is a multi-step process, involving local invasion, circulation, extravasation, colonization and outgrowth of metastatic foci [98]. To identify the steps of this metastatic cascade that requires SMARCE1 activity, we examined the effect of SMARCE1 knockdown on the ability of tumor cells to survive circulation and colonize lungs by using an experimental metastasis model. LM-EV and LM-SMARCE1-KD cells $\left(5 \times 10^{5}\right)$ were injected into the left lateral tail-vein of 5-wk old female NSG mice. Tumor cells in the bloodstream and lung tissues were examined at various time after injection (Figure 2-2A). As expected, the number of circulating tumor cells in blood decreased over time. Interestingly, at any given time point, the number of LM-EV cells in bloodstream was significantly higher than that of the LM-SMARCE1-KD cells (Figure 2-2A). At 72 hours post tail vein injection, we analyzed whole lung lobes under fluorescent microscope and observed red fluorescent tumor cells in lungs of mice inoculated with LM-EV cells but not in mice with LM-SMARCE1-KD cells (Figure 2-2B). Four weeks post injection, a lower number of tumor foci were observed in lungs of mice inoculated with LM-SMARCE1-KD cells compared to mice with LM-EV cells (Figure 2-2C). Together, these results suggest that SMARCE1 knockdown diminishes the ability of tumor cells to survive circulation, thus leading to an overall reduction of lung metastatic foci in mice injected with SMARCE1 knockdown cells.

\section{SMARCE1 knockdown sensitizes tumor cells to anoikis}

Our in vivo experiment results implicate SMARCE1 as an essential contributor in distant metastasis of breast cancer cells by promoting the survival of circulating tumor cells. However, it is unclear whether SMARCE1 plays a role in early metastatic events such as cell migration and invasion. To examine the effect of SMARCE1 knockdown on the migratory and invasive potential of tumor cells, we performed Boyden Chamber Transwell assays. Cells were plated in uncoated Boyden chambers to measure their migratory potential, and in matrigel coated chamber to determine their invasiveness. As showed in Figure 2-3A, SMARCE1 knockdown in LM cells significantly increased cell migratory potential, but showed no significant effect on cell invasiveness. This observation suggests that SMARCE1 activity is dispensable for cell migration. Next, we examined the effect of SMARCE1 knockdown on cell sensitivity to anoikis by monitoring cell viability after cultured in dishes coated with PolyHEMA to prevent attachment. As showed in Figure 2-3B, SMARCE1 knockdown in LM cells substantially reduced the number of viable cells cultured in suspension. This result is consistent with our results from in vivo experiments and confirms that SMARCE1 plays a key role in protecting LM cells against anoikis, a form of cell death induced by detachment.

To determine whether SMARCE1-mediated anoikis resistance ability can be extended to other breast cancer cell lines, we examine the effect of SMARCE1 
A

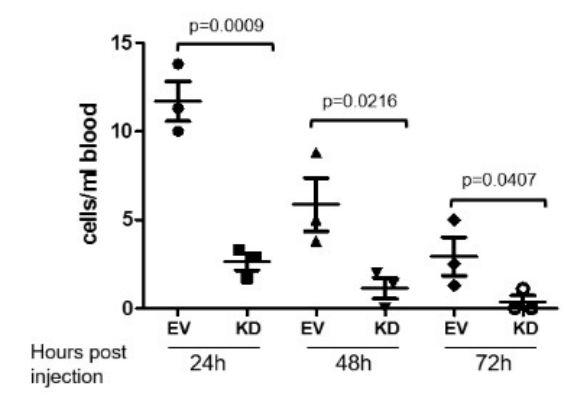

B

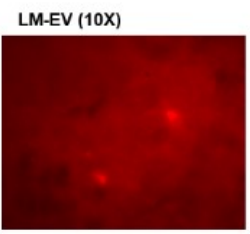

C

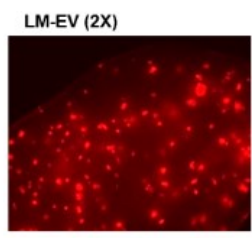

LM-SMARCE1-KD (2X)
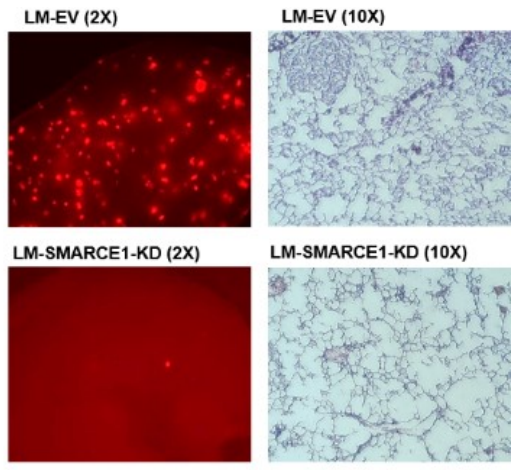

LM-SMARCE1-KD (10X)
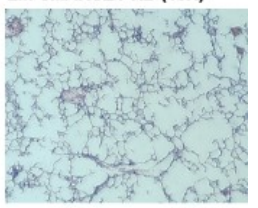

$\mathrm{p}=0.0014$

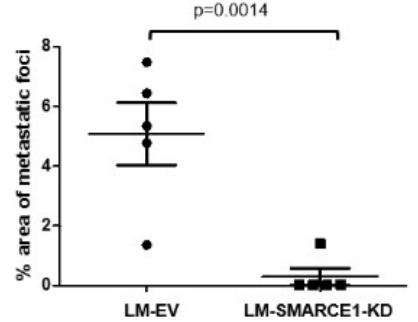

Figure 2-2. SMARCE1 knockdown reduces lung colonization of tumor cells inoculated through tail veins.

A. Number of circulating tumor cells in blood collected at various time points after tail vein injection in NSG mice. B. Fluorescent tumor cells in lungs of NSG mice $72 \mathrm{~h}$ after tail vein injection. Representative images of five lungs for each group were shown. $\mathbf{C}$. Fluorescent tumor foci in the left lung lobes of NSG mice 4-wk after tail vein injection of tumor cells. The area of tumor foci on the dorsal surface of the left lung lobe was quantified by ImageJ. 

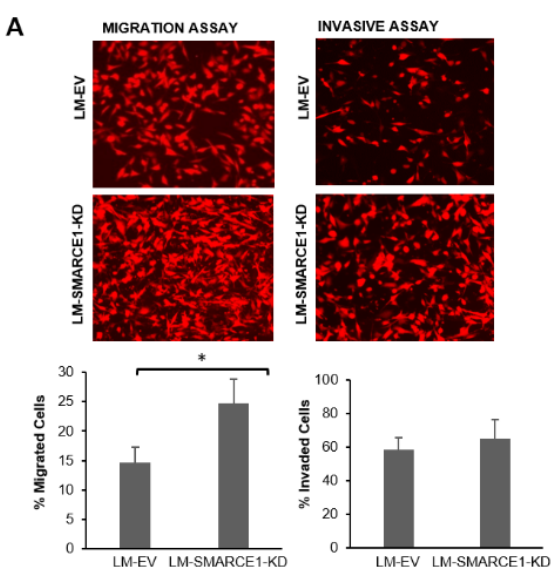

B

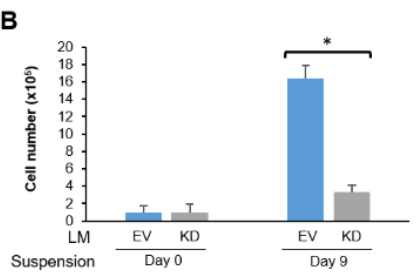

E
C

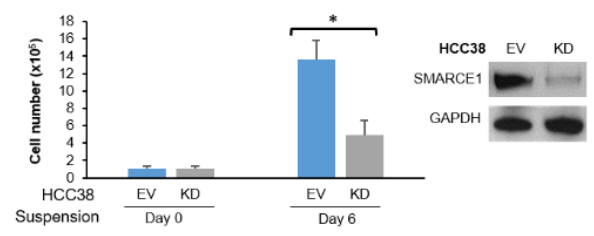

D
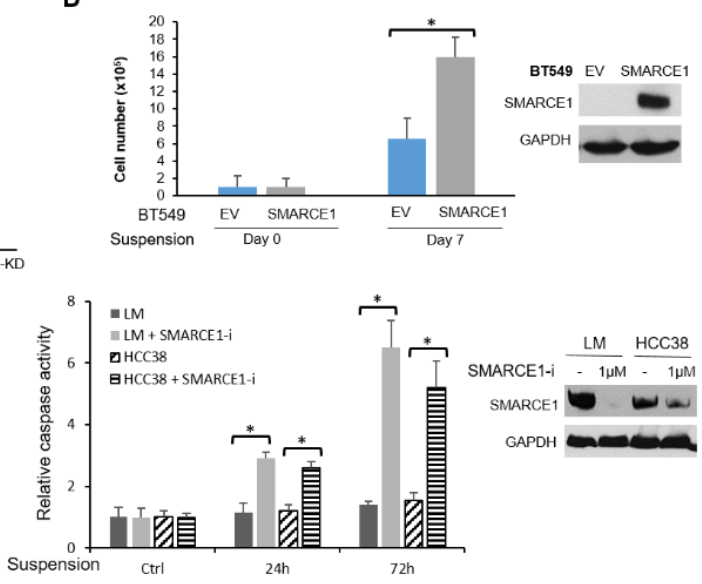

Figure 2-3. SMARCE1 knockdown promotes cell migration, but sensitize cells to anoikis.

A. Effect of SMARCE1 knockdown in LM cells on cell migration and invasion. Boyden chambers, uncoated or coated with matrigel were used to measure cell migratory and invasive potential, respectively. Percent migration/invasion was calculated as per manufacturer's protocol B. Effect of SMARCE1 knockdown in LM cells on viability of cells cultured in dishes coated with PolyHEMA to prevent adhesion. C. Effect of SMARCE1 knockdown in HCC38 cells. D. SMARCE1 overexpression in BT549 cells. E. Blocking SMARCE1 expression by siRNA enhanced detachment-induced caspase activation in LM and HCC38 cells. Accell SMARTpool of SMARCE1-siRNA $(1 \mu \mathrm{M})$ were delivered into cells $48 \mathrm{~h}$ prior to detachment. Caspase activities were determined at indicated time point after cell detachment by using the Caspase-Glo 3/7 assay kit. $* \mathrm{p}<0.05, \mathrm{n}=6$, Student T-test. 
knockdown in HCC38 (Figure 2-3C), a triple-negative breast cancer cell line. We found that SMARCE1 knockdown resulted in significant reduction of the viability of cells in suspension culture. In addition, we examined the effect of SMARCE1 overexpression in BT549, a triple-negative breast cancer cell line that lacks SMARCE1 expression due to a biallelic inactivating mutation that causes a frameshift [99]. As expected, no SMARCE1 mRNA or protein was detected in BT549 cells (Figure 2-3D). Enforced SMARCE1 overexpression in BT549 substantially increased the viability of cells in suspension culture. To validate the observations with SMARCE1-shRNA, we examined the effect of SMARCE1 siRNA (Accell SMARTpool, GE Dharmacon) or control oligonucleotides on anoikis in LM and HCC38 cell lines. As showed in Figure 2-3E, SMARCE1 siRNA (SMARCE1-i) treatment resulted in decreased expression of SMARCE1 protein and enhanced activation of caspase 3/7 in cells under suspension culture. These results demonstrate that SMARCE1 plays role in anoikis resistance of breast cancer cells.

\section{SMARCE1-mediated anoikis resistance requires activation of PTK2, MAPK and AKT pathways}

To gain insights into the molecular mechanisms underlying SMARCE1-mediated anoikis resistance, we sought to identify signaling pathways regulated by SMARCE1 in detached cells by using chemical inhibitors of several signaling pathways that have been linked to anoikis resistance of cancer cells [100, 101]. As showed in Figure 2-4A, inhibitors of focal adhesion kinase (PTK2/FAK), MAPK and AKT significantly reduced the viability of LM-EV cells in suspension culture. In contrast, these inhibitors showed no significant effect on LM-SMARCE1-KD cells. The same experiments were performed with BT549-EV and BT549-SMARCE1 cells and the result showed that SMARCE1mediated increase of cell viability was effectively abolished by inhibitors of PTK2, MAPK and AKT (Figure 2-4B). In agreement with the observed effects of chemical inhibitors on cell viability, immunoblotting analysis showed that LM-EV cells in suspension culture had higher levels of PTK2 protein, MAPK phosphorylation and AKT phosphorylation compared to LM-SMARCE1-KD cells (Figure 2-5A). In addition, elevated expression of the pro-apoptotic protein BIM/BCL2L11, a marker of onset of anoikis in breast cancer cells, was detected in suspension cultures of LM-SMARCE1-KD cells, but not in LM-EV cells (Figure 2-5A). Consistently, higher levels of PTK2 protein and $\mathrm{AKT}$ phosphorylation, concomitant with lower levels of BIM protein, were observed in HCC38-EV cells as compared to HCC38-SMARCE1-KD cells in suspension culture (Figure 2-5B). However, MAPK phosphorylation was not affected by cell detachment or SMARCE1 knockdown in the HCC38-EV cells. In addition, elevated expression levels of PTK2 protein, MAPK phosphorylation and AKT phosphorylation, but diminished levels of BIM, were detected in BT549-SMARCE1 cells in comparison to control BT549-EV cells under suspension culture (Figure 2-5C). Together, these results suggest that activation of PTK2, MAPK and AKT survival pathways are critical for breast cancer cells to survive anoikis, and SMARCE1 activity is required for the activation of these pro-survival pathways in detached cells. 
A

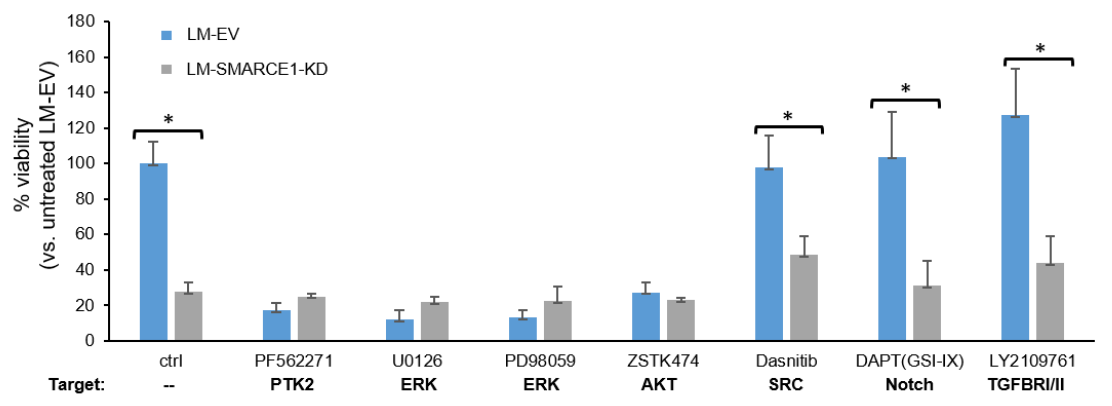

B

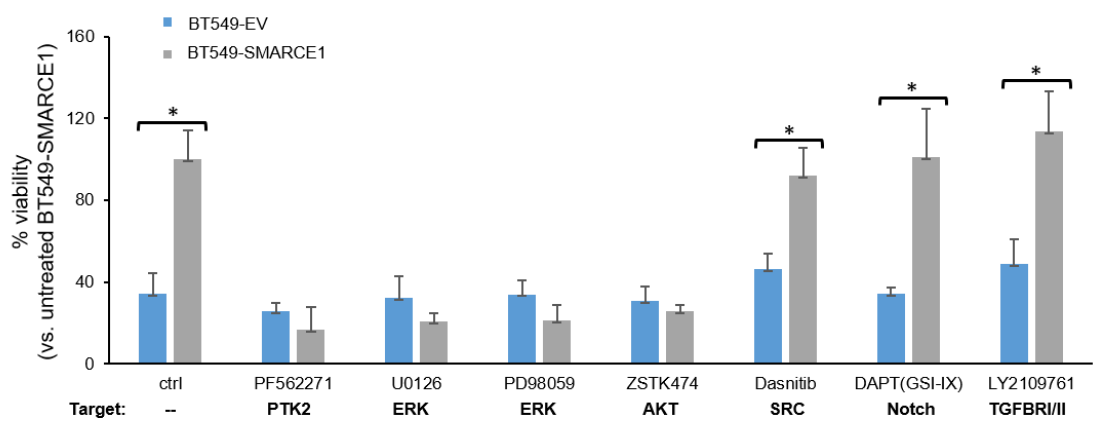

Figure 2-4. SMARCE1-mediated anoikis resistance requires activation of PTK2, MAPK and AKT pathways.

A. Viability of LM-EV and LM-SMARCE1-KD cells cultured in PolyHEMA-coated dishes in the absence or presence of indicated inhibitors. B. Viability of BT549-EV and BT549-SMARCE1 cells cultured in PolyHEMA-coated dishes in the absence or presence of indicated inhibitors. For all experiments, viable cells were counted after 7 days in

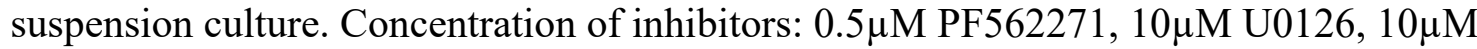

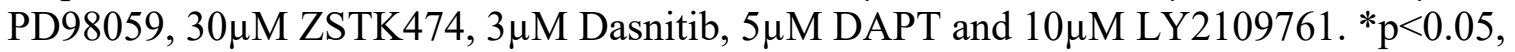
$\mathrm{n}=3$, Student T-test. 

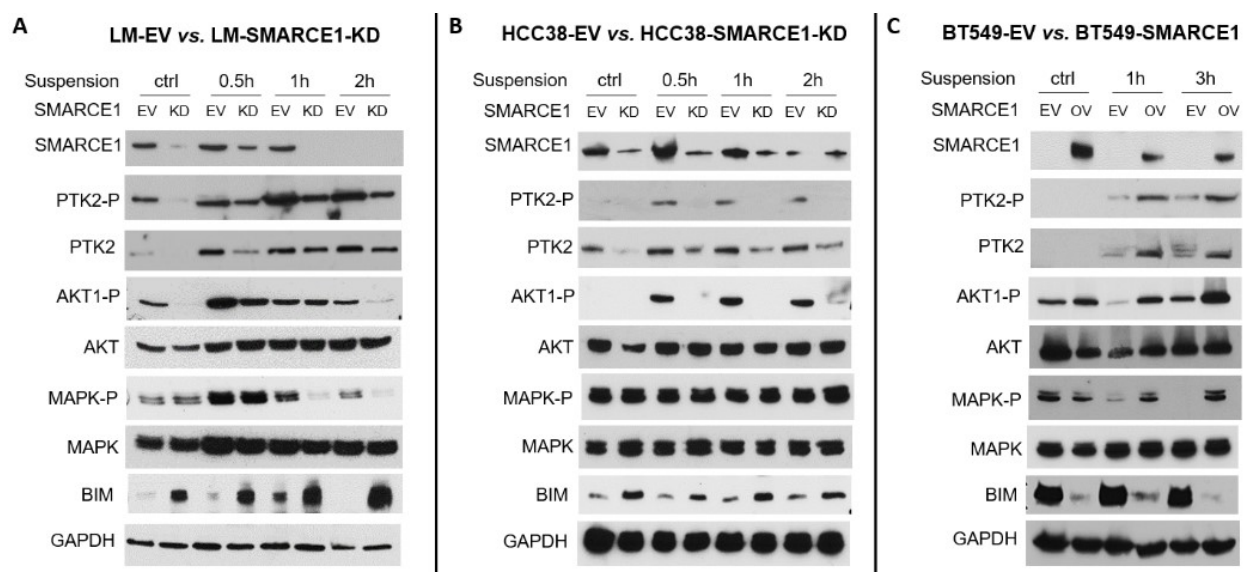

Figure 2-5. SMARCE1-dependent activation of PTK2, MAPK and AKT pathways by cell detachment.

A. Effect of SMARCE1 knockdown in LM cells on detachment-induced activation of pro-survival (FAK/PTK2, MAPK and AKT) and pro-apoptotic (BIM) signaling pathways. B. Effect of SMARCE1 knockdown in HCC38 cells. C. Effect of SMARCE1 overexpression in BT549 cells. The levels of protein expression and phosphorylation were determined by immunoblotting assays. GAPDH was included as a loading control. 


\section{SMARCE1 collaborates with HIF1A to activate PTK2 transcription in detached cells}

Aberrant activation of PTK2, a non-receptor tyrosine kinase involved in focal adhesion, has been recognized as a key mediator of anoikis resistance in variety of tumor cells $[100,102]$. For example, PTK2 activation has been detected in circulating tumor cells isolated from $90 \%$ of patients with metastatic breast tumors, implicating a role of PTK2 in breast cancer metastasis [103]. Therefore, we sought to examine whether SMARCE1 regulates PTK2 expression in detached cells. Based on immunoblotting and qPCR analysis, we found that cell detachment induced PTK2 expression at both the protein level (Figure 2-5) and mRNA level (Figure 2-6A) in a SMARCE1-dependent manner in LM, HCC38 and BT549 cells. This observation suggests that SMARCE1 is required for $P T K 2$ gene activation on a transcriptional level in detached cells.

By inspecting the transcription factor binding consensus sequences in the proximal promoter region of $P T K 2$ gene (Figure 2-6B), we found a potential HIF1A/ARTN binding site located in the binding region of SWI/SNF subunits (SMARCA4 and SMARCB1) identified by ENCODE TFBS ChIP-seq analysis [104]. Normoxic HIF1A activation has been linked to anoikis resistance in breast cancer cells $[103,105]$. In addition, SWI/SNF complexes were reported to play a role in modulating HIF1A-mediated transcription activation in cells under hypoxia [79, 106]. Therefore, we speculated that SMARCE1 regulates PTK2 gene transcription through HIF1A in detached cells. Using CHIP-qPCR assays, we showed that that cell detachment induced recruitment of SMARCE1, HIF1A and BRG1/SMARCA4 to the PTK2 promoter region (-874 to $-575 \mathrm{bp}$ ) in LM-EV cells, which was abolished by SMARCE1 knockdown (Figure 2-6B). In addition, using reciprocal immunoprecipitation assays, we detected HIF1A and SMARCE1 proteins in the immunoprecipitated SMARCE1 and HIF1A complexes, respectively (Figure 2-6C). These results suggest that SMARCE1 activity is required for HIF1A-mediated PTK2 transcription activation in detached cells.

Since the SWI/SNF complex is involved in nucleosomal remodeling we sought to examine changes in the nucleosomal positioning on the PTK2 promoter. Nucleosome scanning assay (NUSA) was performed to examine the consequence of SMARCE1 and HIF1A binding to the PTK2 promoter in detached cells. We found that cell detachment induced nucleosomal disassembly on the PTK2 promoter region bound by HIF1A and SMARCE1, indicated by increased sensitivity of PTK2 promoter to dsDNase digestion, which resulted in decreased amount of DNA detected by qPCR (Figure 2-7). However, cell detachment did not increase dsDNase sensitivity of PTK2 promoter in LM-

SMARCE1-KD cells (Figure 2-7B, right panel). These observations support the role of SMARCE1 in chromatin remodeling of PTK2 promoter and HIF1A-mediated PTK2 transcriptional activation in detached cells. 
A

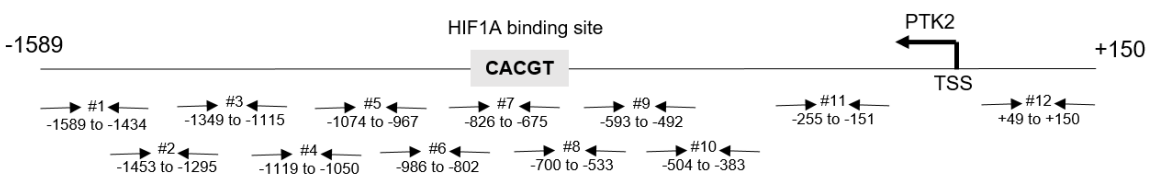

B
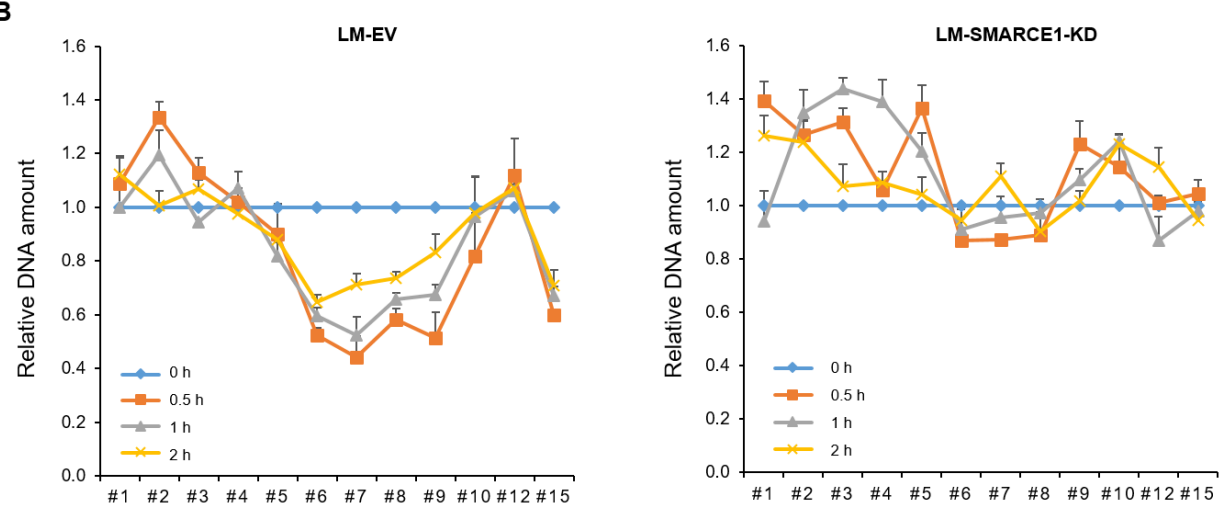

Figure 2-6. SMARCE1 collaborates with HIF1A to activate PTK2 transcription in detached cells.

A. Detachment-induced PTK2 mRNA expression is abolished by SMARCE1 knockdown in LM and HCC38 cells, whereas enhanced by SMARCE1 overexpression in BT549 cells. B. Detachment-induced recruitment of HIF1A and SMARCA4 to the promoter region of PTK2 is diminished by SMARCE1 knockdown in LM cells. Chromatin binding of HIF1A, SMARCE1 and SMARCA4 was measured by ChIP-qPCR assay. Average fold enrichment of PTK2 promoter DNA by indicated antibodies ( $v s$. control IgG) from three independent experiments are presented. C. Detachment induces interaction between SMARCE1 and HIF1A in LM-EV and LM-SMARCE1-KD cells. The protein interaction was examined by Immunoprecipitation/Immunoblotting assays. ${ }^{*} p<0.05$, Student T-test. 
A
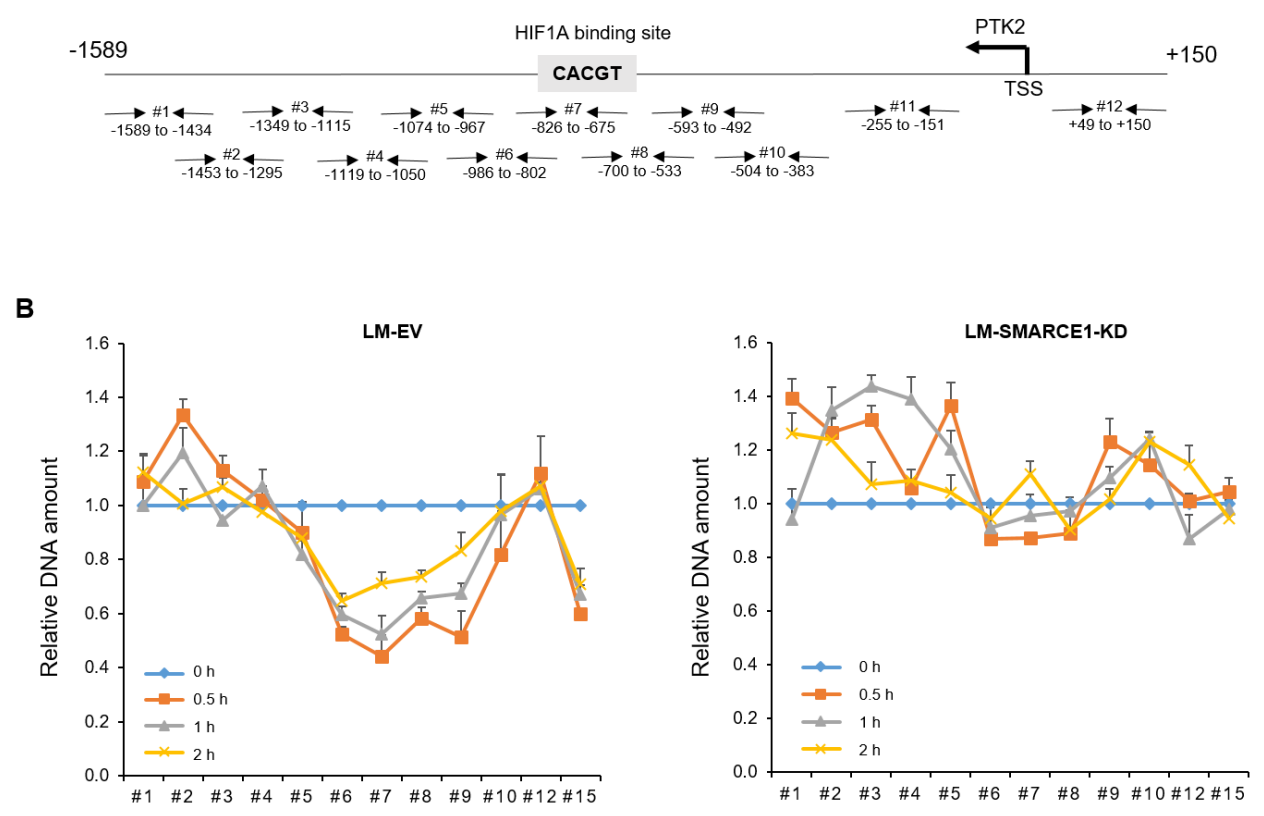

Figure 2-7. SMARCE1 orchestrates chromatin remodeling of PTK2 promoter in detached cells.

A. Overlapping primer designed for the nucleosome scanning assay and their amplicon sizes. The location of each primer set is given relative to the transcription start site (TSS). B. Significant nucleosomal displacement is observed on PTK2 promoter (from -802 to 492 bp, encompassing HIF1A binding site) in LM-EV cells after 0.5, 1 and 2 hours under suspension culture (vs. adherent cells), but not in LM-SMARCE1-KD cells. Results from three independent experiments were presented as means $\pm \mathrm{SE}$. 


\section{HIF1A activates $P T K 2$ transcription and downstream activation of survival pathways in detached cells}

To confirm that HIF1A-mediated PTK2 transcription is critical for breast cancer cell to survive anoikis, we examined the effect of HIF1A knockdown on cell sensitivity to anoikis and detachment-induced activation of PTK2, MAPK and AKT. HIF1A knockdown in MDA-MB-231 cells effectively dampened detachment-induced HIF1A protein accumulation and $P T K 2$ mRNA expression (Figure 2-8A and B), and reduced the number of viable cells in suspension culture (Figure 2-8C). Immunoblot analysis showed levels of PTK2, phosphorylated-PTK2 (pTyr576/577), phosphorylated-MAPK (pThr202/Tyr204) and phosphorylated-AKT (pTyr416) were significantly lower in HIF1A-KD cells than that in control MDA-MB-231-EV cells under suspension culture (Figure 2-8D). Conversely, the pro-anoikis protein BIM was induced only in detached HIF1A-KD cells. To confirm that MAPK and AKT phosphorylation are downstream events of PTK2 activation in detached cells, we examined the effect of PTK2 inhibition by PF562271 on activation of these pathways. As showed in Figure 2-8E, pre-treatment with PF562271 effectively abolished detachment-induced phosphorylation of PTK2, MAPK and AKT. Taken together, these results suggest that HIF1A-mediated PTK2 transcription activation plays a key role in protecting cells against anoikis by sustaining the activation of survival signaling pathways (MAPK and AKT), as well as suppressing pro-apoptotic signaling (BIM).

To corroborate the role of PTK2 in SMARCE1-mediated anoikis resistance, we examined the effects of PTK2 overexpression and PTK2 knockdown on anoikis sensitivities of LM-SMARCE1-KD and BT549-SMARCE1 cells, respectively. We found that PTK2 overexpression effectively rescued LM-SMARCE1-KD cells from anoikis, which is indicated by increased number of viable cells and reduced caspase activation (Figure 2-9A, B, C, and D). Conversely, PTK2 knockdown abolished SMARCE1mediated protection of BT549 cells against anoikis (Figure 2-9E and F).

\section{SMARCE1 knockdown sensitizes non-tumorigenic mammary epithelial cells to anoikis}

To examine whether the SMARCE1-dependent anoikis resistance occur at early stage of tumorigenesis, we examined the effect of SMARCE1 knockdown on anoikis sensitivity of 184B5, a mammary epithelial cell line immortalized by benzo(a)pyrene exposure [107]. Previous studies showed that 184B5 was not tumorigenic in immunosuppressed mice, but 184B5 does not form colonies in semisolid medium [107]. Therefore, 184B5 represents cells at early stage of tumorigenesis. We found that SMARCE1 inhibition in 184B5 cells, by either stable knockdown through shRNA expression (Figure 2-10A) or transient siRNA delivery (Figure 2-10B), blocked detachment-induced PTK2 upregulation (Figure 2-10C) and sensitized cells to anoikis, indicated by reduced cell numbers and increased caspase activation (Figure 2-10A and $\mathbf{B}$, respectively). These observations suggest that SMARCE1/PTK2-mediated anoikis resistance is an early event of tumorigenesis. 


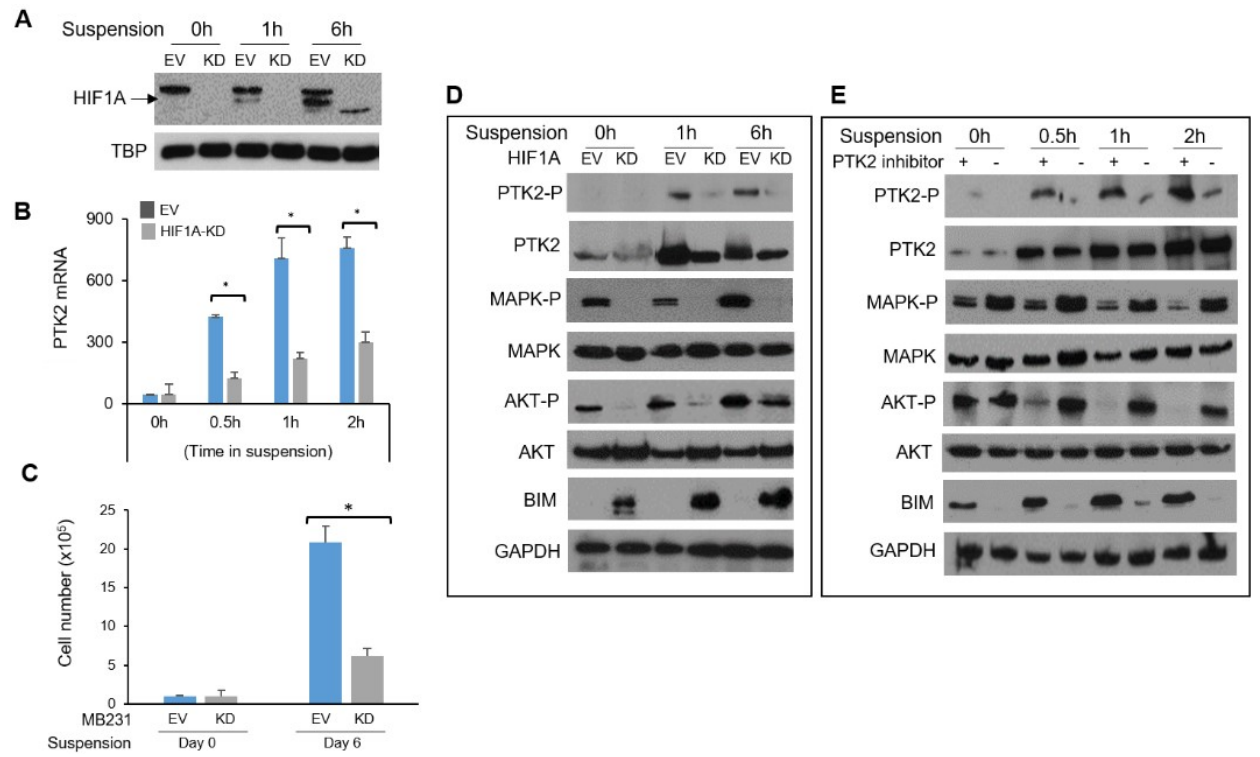

Figure 2-8. HIFIA activates PTK2 transcription and downstream survival pathways in detached cells.

A. Detachment induced HIF1A protein accumulation is eliminated by HIF1A knockdown in MDA-MB-231 cells. B. HIF1A knockdown abolishes detachment induced PTK2 mRNA expression in MDA-MB-231 cells. C. HIF1A knockdown sensitizes MDA-MB231 cells to anoikis. Viable cells in suspension culture for 10 days were counted. $\mathbf{D}$. Effect of HIF1A knockdown in MDA-MB-231 cells on detachment-induced activation of pro-survival (PTK2, MAPK and AKT) and pro-apoptotic (BIM) signaling pathways. E. Effect of PTK2 inhibition in MDA-MB-231 cells on detachment-induced activation of pro-survival and pro-apoptotic signaling pathways. The mRNA levels were measured by qPCR. The levels of protein expression and phosphorylation were determined by immunoblotting assays with GAPDH included as a loading control. ${ }^{*} p<0.05$, Student Ttest. 
A

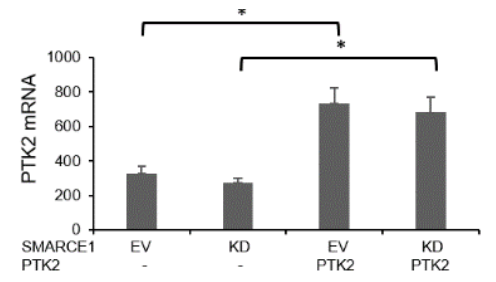

C

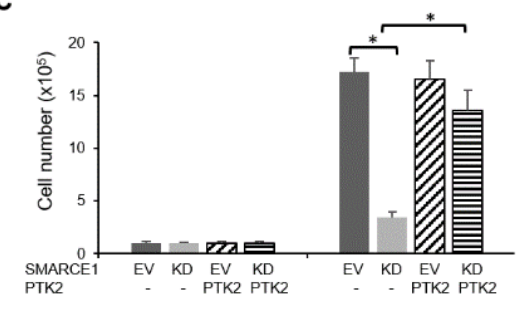

E

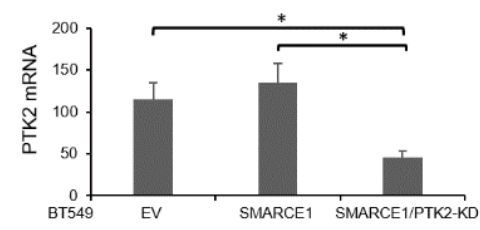

B

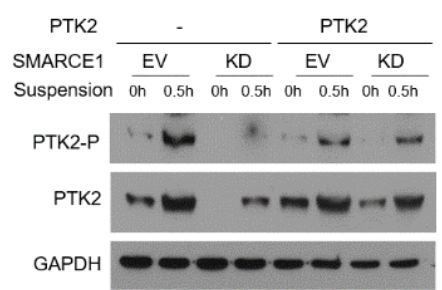

D

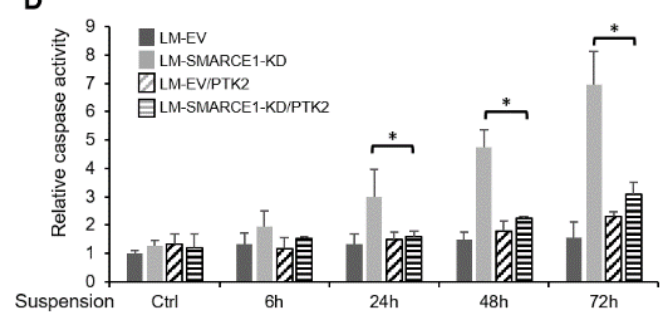

$\mathbf{F}$

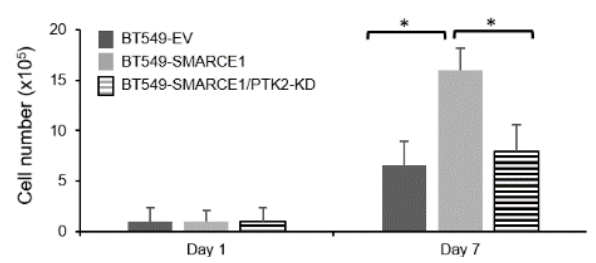

Figure 2-9. PTK2 activation is essential for SMARCE1-mediated anoikis resistance in breast cancer cells.

A. Expression levels of PTK2 mRNA in LM derivatives. B. PTK2 protein expression and phosphorylation in LM derivatives cultured in PolyHEMA-coated dishes. Intensities of immunoblotting signals were quantified by ImageJ and the average of normalized intensities (vs. GAPDH) from three independent experiments were presented. C. Effect on PTK2 overexpression on viability of LM derivatives cultured in PolyHEMA-coated dishes. D. Effect on PTK2 overexpression on caspase activation in LM derivatives cultured in PolyHEMA-coated dishes. Caspase activity was normalized to cell numbers and results from three independent experiments were presented as means $\pm \mathrm{SE}$. E. Expression levels of PTK2 mRNA in BT549 derivative. F. Effect of PTK2 knockdown on viability of BT549 derivative cultured in PolyHEMA-coated dishes. ${ }^{*} \mathrm{p}<0.05$, Student T-test, $\mathrm{LM}=$ lung metastatic derivative of MDA-MB-231 cells. 
A

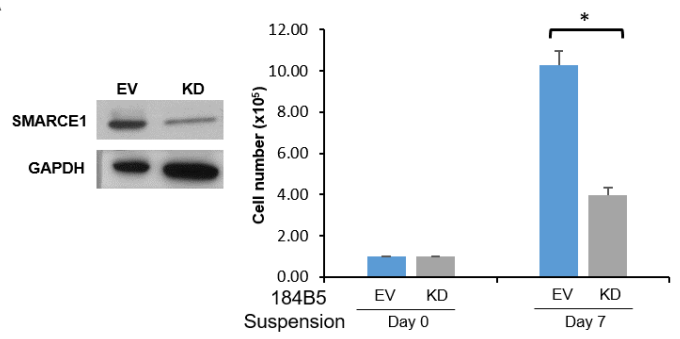

C

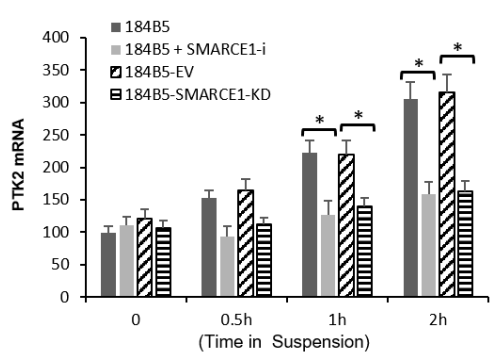

B

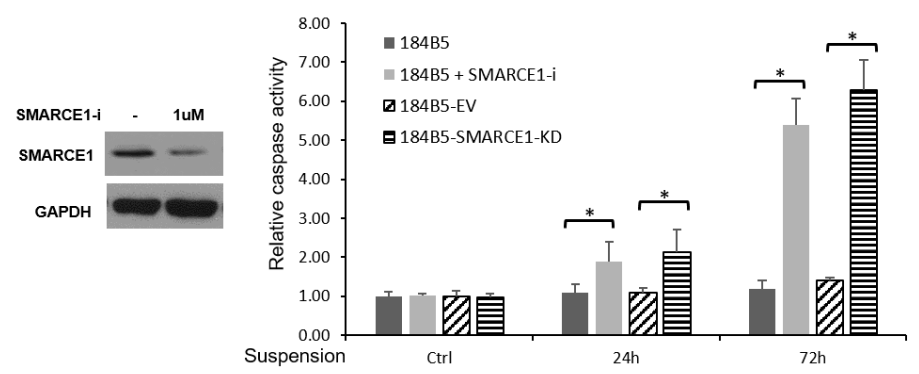

Figure 2-10. Blocking SMARCE1 expression sensitizes non-tumorigenic mammary epithelial cells to anoikis.

A. Effect of SMARCE1 knockdown by stable shRNA expression on viability of 184B5 cells cultured in PolyHEMA-coated dishes. B. Effect of stable shRNA expression (SMARCE1-KD) and SMARCE1-siRNA delivery (SMARCE1-i) on caspase activation in 184B5 cells cultured in PolyHEMA-coated dishes. Caspase activity was measured by using the Caspase-Glo 3/7 assay kit and normalized to cell numbers. The results from three independent experiments were presented as means \pm SE. C. Detachment-induced PTK2 mRNA expression is abolished by SMARCE1-KD or SMARCE1-i in 184B5 cells. mRNA levels were measured by qPCR. * ${ }^{*}<0.05, \mathrm{n}=3$, Student T-test. 


\section{Higher SMARCE1 and PTK2 expression is associated with poor prognosis of patients with basal-like or luminal B subtype of breast tumors}

Having established a role of SMARCE1 in regulating metastatic potential of breast cancer cells through the HIF1A/PTK2 pathway, we sought to elucidate the clinical relevance of this finding by examining the relationship between the SMARCE1 and PTK2 expression and the clinical outcomes of breast cancer patients. Analyzing expression data of 3554 breast tumor samples collected by an online Kaplan-Meier survival analysis tool [108], we found that higher expression of SMARCE1 or PTK2 is associated with shorter interval of relapse free survival (RFS) of breast cancer patients (Figure 2-11A). However, this RFS was not correlated when tumor samples were grouped according to ESR1 expression or intrinsic PAM50 subtype markers. Next, we examined the relationship between the expression of SMARCE1 and PTK2 in breast tumors in The Cancer Genome Atlas (TCGA) database [109]. No significant correlation between SMARCE1 and PTK2 expression was detected when data from all breast tumors $(n=825)$ were included in the analysis. However, after tumors were stratified according to the intrinsic PAM50 subtype markers, a positive correlation between SMARCE1 and PTK2 expression was detected in the luminal B and basal-like cohorts (Figure 2-11B), but not in the human epidermal growth factor receptor 2, ERBB2-enriched or Luminal A cohorts. Together, these observations suggest that higher expression of SMARCE1 and PTK2 increases risk of tumor relapse, and SMARCE1 likely plays a key role in regulating PTK2 expression in luminal B and basal-like tumors.

\section{Genomic alterations contribute to deregulation of SWI/SNF complexes in breast cancer}

Despite the prevalent notion that SWI/SNF complexes are tumor suppressors [110], emerging evidence suggests that genetic alterations of subunit genes can produce aberrant SWI/SNF complexes with oncogenic function [111]. These findings prompted us to examine the nature and frequency of genetic alterations in SMARCE1 and other SWI/SNF subunits specifically in breast cancer by using the TCGA database. The result revealed that approximately $30 \%$ breast tumors harbor genetic alterations in one or more of the genes encoding SWI/SNF subunits (Figure 2-11C). Interestingly, gene amplification is the most frequent genetic event for multiple SWI/SNF subunits in breast cancer, rather than inactive mutations that were reported in other tumors [88]. SMARCE1 is the second most frequently amplified gene among all the SWI/SNF subunits. This finding implicates an oncogenic role of SWI/SNF complexes in human breast cancer. If a SWI/SNF subunit plays a critical role in promoting progression of breast cancer, we speculate a positive correlation between its expression and poor prognosis of breast cancer patients. Therefore, we examined the relationship between the expression of each SWI/SNF subunit and interval of RFS of breast cancer patients using the online KaplanMeier survival analysis tool [108]. This analysis revealed higher expression of 9 (out of 31) SWI/SNF subunits (i.e., BCL7C, BRD7, ACTL6A, SMARCE1, SMARCA5, SMARCAL1, SMARCA4, SS18 and SMARCC1) is associated with shorter interval of RFS of breast patients (LogRank $\mathrm{p}<0.05, \mathrm{n}=3550)$. Overall the 
A

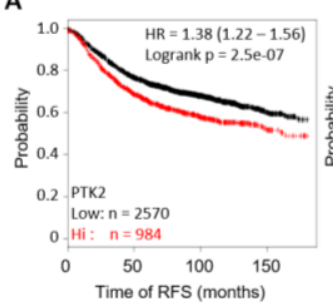

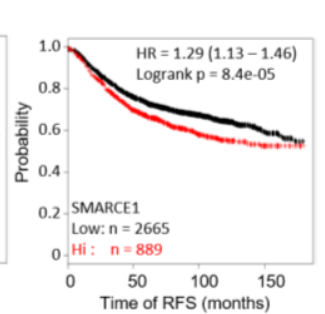

B
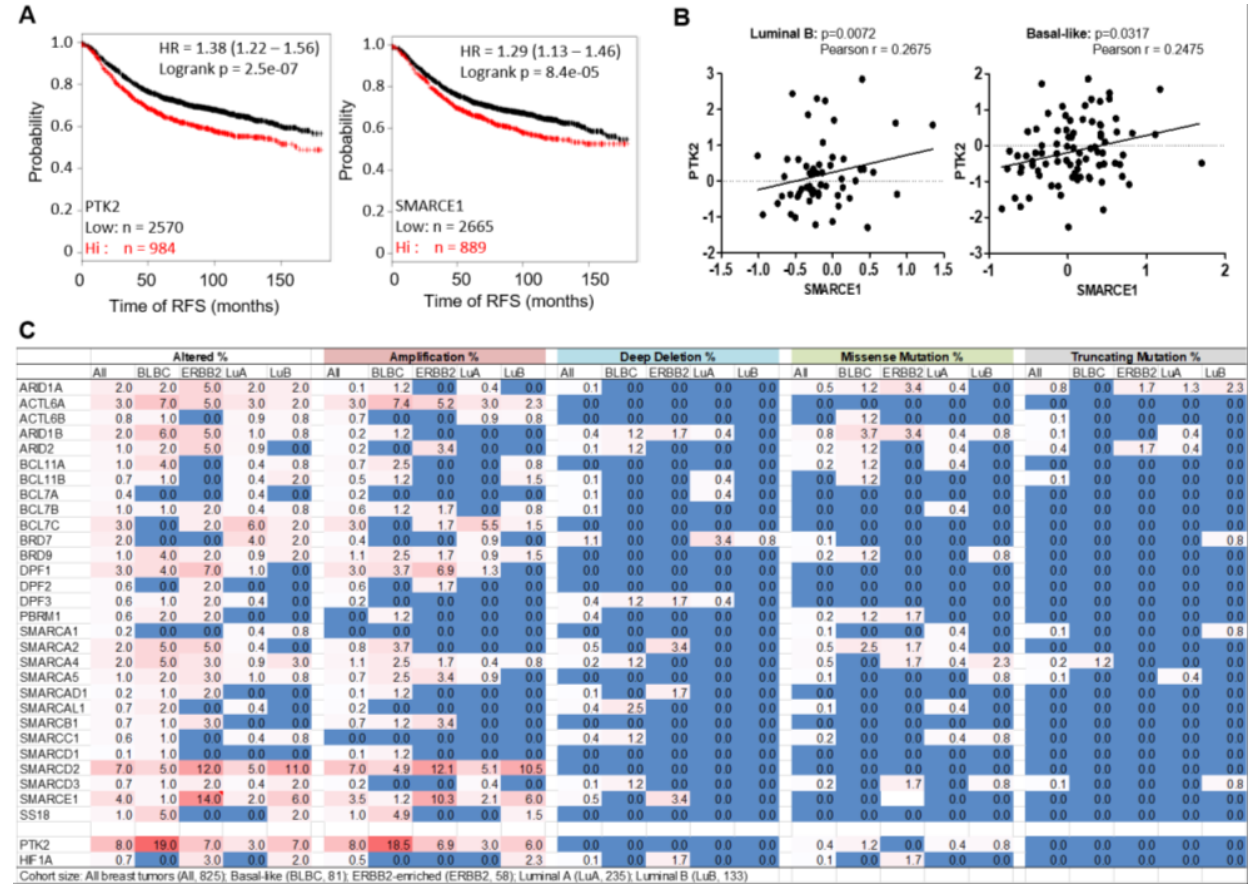

Figure 2-11. Higher SMARCE1 and PTK2 expression is associated with poor prognosis of breast cancer patients.

A. Kaplan-Meier analysis of recurrence-free survival (RFS) of breast cancer patients based on SMARCE1 and PTK2 expression using dataset of 3554 breast tumor samples (http://kmplot.com/analysis/). To construct Kaplan-Meier curves all percentiles between the lower and upper quartiles were computed and the best performing threshold was used as cutoff to divide the patient cohort. The LogRank test $P$ value was calculated by using RFS up to 15 years as endpoint. B. Correlated expression of SMARCE1 and PTK2 in basal-like and luminal B subtypes of breast cancer according to the TCGA database. Tumors with PTK2 amplification were excluded from the correlation analysis. C. Frequency of genetic alterations of SWI/SNF complex subunits in breast tumors according to the TCGA database. 
results from genetic and expression data analysis suggest that deregulation of SWI/SNF activity by altering expression of core subunits likely plays a key role in promoting breast cancer progression.

\section{Discussion}

Loss of adhesion to extracellular matrix (ECM) triggers a specific form of apoptosis termed anoikis in normal epithelial cells [112], which plays a fundamental role in morphogenesis of the mammary gland ductal system [113]. Circumvention of anoikis contributes to breast tumor development and is a prerequisite for metastases by allowing cells to survive loss or alterations of ECM $[85,100]$. In mammary epithelial cells, the dynamic and magnitude of activation of pro-apoptotic genes (e.g., BIM and BMF) or prosurvival proteins (e.g., PTK2) were shown to play a critical role in determining the fates of detached cells, anoikis or survival [38, 85, 100, 114]. PTK2 is one of the most important signaling proteins recruited into focal adhesions upon cell-ECM contact, where PTK2 is rapidly auto-phosphorylated to enable the recruitment of other scaffold and signaling molecules to activate the downstream cell survival signaling pathways (e.g., PI3K/Akt and MAPK/ERK pathways) $[102,114,115]$. Emerging evidence suggests that PTK2 plays a key role in protecting tumor cells against anoikis by sustaining survival signaling [100, 102]. However, how PTK2 is activated in detached cells has not been definitively resolved. Our studies revealed a key role of SMARCE1 in promoting survival of detached cells by facilitating rapid PTK2 transcription activation through HIF1A, the master regulator of hypoxia responsive genes. Multiple kinases were reported to activate PTK2 through phosphorylation at Tyr576/577 in detached cells, including SRC family kinases and PTK6 [100, 116]. We found that SRC inhibition by Dastinib, a SRC family tyrosine kinase inhibitor, had no effect on viability of LM and BT549 cells under suspension. This observation suggests that PTK2 phosphorylation in detached breast cancer cells is likely sustained by an SRC-independent mechanism.

HIF1A activation under normal oxygen tension has been linked to anoikis resistance of breast cancer cells driven by the ERBB2 oncogene [105], and circulating breast tumor cells from patients with metastatic breast cancer [103]. SWI/SNF complexes were shown to regulate transcription activation of a subset of HIF1A target genes under hypoxia [106]. However, it is unclear whether the SWI/SNF complex is required for HIF1A transcription activity in detached cells under normal oxygen tension. Our study provides evidence suggesting an essential role of SMARCE1-based chromatin remodeling activity in breast cancer metastasis by facilitating HIF1A mediated PTK2 transcription activation in detached cells under normoxia. Further study is warranted to evaluate the anti-metastastic potential of approaches targeting SMARCE1-HIF1A interaction.

According Project Achilles, the database which performed genome-wide pooled shRNA screens across hundreds of cancer cell lines to identify genes essential for tumor cell proliferation, SMARCE1 knockdown showed limited effect on proliferation of breast cancer cells [117]. Consistently, we found that SMARCE1 knockdown had no 
significantly effect on proliferation of breast cancer cell lines -MDA-MB-231 and HCC38; in vitro and growth of orthotopic xenografts derived from MDA-MB-231 cells. However, SMARE1 knockdown did significantly enhanced anoikis sensitivity of breast cancer cells. These findings suggest that SMARCE1 is dispensable for proliferation of breast cancer cells under normal growth condition, but required for cells to survive anoikis. Whether SMARCE1 plays a role in protecting cells against stress-induced cell death in general requires further investigation.

In contrast to colorectal cancer, melanoma or lung cancers which frequently harbor inactive mutations of SWI/SNF subunits, breast tumors have a relatively low frequency of inactive mutations $[88,92,93]$. By inspecting the TCGA database we found that $\sim 30 \%$ breast tumors harbor genetic alterations (e.g., gene amplification, deletion and mutation) in one or more of the SWI/SNF subunits, with gene amplification being the most frequent event. This finding implicates an oncogenic role of SWI/SNF complexes in breast cancer. Consistently, we found that higher expression of several SWI/SNF subunits was associated with shorter interval of RFS of breast cancer patients. Our findings are likely relevant to basal-like and luminal B breast tumors subtypes, due to a positively correlated expression profile between SMARCE1 and PTK2. In conclusion, this study establishes a novel role of SMARCE1 in regulating metastatic potential of breast cancer cells by promoting survival of detached cells through the HIF1A/PTK2 pathway. 


\section{CHAPTER 3. ROLE OF BHLHE40 IN BREAST CANCER METASTASIS}

\section{Introduction}

One in every eight women in the United States will be diagnosed with breast cancer over the course of her lifetime [118]. An estimated 266,120 new cases are expected, and 40,920 women are expected to die from breast cancer in 2018 in the United States [118]. Distant metastasis is the major cause of breast cancer related deaths. Hypoxia has been recognized as a primary driving force of distant metastasis of breast cancer [19, 49, 119-122]. Among hypoxia responsive genes are both promoting and suppressive factors for malignant progression. It is unclear how the expression and activities of metastasis-promoting factors are preferentially augmented in metastatic tumors. A large body of studies have focused on elucidating the molecular mechanisms by which hypoxia enables cancer cells to survive a variety of stresses imposed during the metastatic cascade, including nutrient depletion, loss of attachment and deprivation of growth factors.

Hypoxia-induced exosomic secretion of cytokines and growth factors plays a key role in promoting metastasis through both tumor autonomous and non-autonomous mechanisms $[123,124]$. Exosomes are microvesicles $(40-130 \mathrm{~nm})$ constitutively released by a variety of cells into the extracellular environment to promote cell-to-cell communication [125]. Tumor cells have been reported to utilize exosomes to transfer nucleotides, lipids and proteins into surrounding cells or cells in distant metastatic niches $[125,126]$. Hypoxia is known to markedly increase the number of secreted exosomes, as well as alter the contents of exosomes [127]. However, our understanding of the regulation of exosome secretion is rudimentary.

Although the cellular response to hypoxia is mainly controlled by two basic helixloop-helix transcription factors, HIF1A and EPAS1/HIF2A, the outcomes of hypoxia response are modified by other transcription regulators that are regulated by hypoxia or interact with HIF1A or EPAS1 [128, 129]. This study focuses on the role of a basic helix loop helix transcription factor BHLHE40 (also known as DEC1/ BHLHB2/ SHARP2/ STRA13) in metastasis of breast cancer. BHLHE40 expression is directly activated by HIF1A in a variety of tumor cells under hypoxia [130, 131]. High BHLHE40 expression has been linked to activation of hypoxia response pathway, elevated metastatic potentials and poor prognosis of various types of tumors, including hepatocellular carcinoma, pancreatic cancer and invasive breast cancer [132-134]. It is reported that BHLHE40 binds to the E-box elements and regulates the expression of genes associated with circadian rhythm, cell differentiation, cell senescence, lipid metabolism, DNA damage response and immune response [135-138].

However, the action mechanism and downstream targets of BHLHE40 in breast cancer cells are largely unknown. In this study, we provide evidence that BHLHE40 is a pro-metastasis factor in breast cancer cells that promotes tumor cell survival and 
migration by modulating exosomic secretion of HBEGF, a heparin binding epidermal growth factor.

\section{Methods}

\section{Cell culture}

Breast cancer MDA-MB-231 and MCF7 cells were obtained from ATCC (Manassas, VA, USA) and maintained in Minimal Essential Medium (ThermoFisher Scientific, Rockford, IL, USA) supplemented with 10\% fetal bovine serum (FBS), 200 $\mathrm{U} / \mathrm{ml}$ of penicillin-streptomycin and $0.5 \mu \mathrm{g} / \mathrm{ml}$ amphotericin B (Cellgro, Manassa, VA, USA). A lung metastatic derivative of MDA-MB-231 (LM) and a tamoxifen-resistant derivative of MCF7 (TR) were established as described previously [139, 140]. A stable line (BHLHE40-KD) expressing a short hairpin RNA against BHLHE40 (TRCN0000232187, Sigma-Aldrich, St Louis, MO, US) were generated by lentiviral transduction and selected in medium containing $2 \mu \mathrm{g} / \mathrm{ml}$ puromycin (Sigma-Aldrich). A colony of BHLHE40 knockout variant (BHLHE40-KO) of MDA-MB-231 was generated by using the CRISPR/Cas9 all-in-one expression system (HCP221270-CG01-1, GeneCopoeia) and selected in medium supplemented with $500 \mu \mathrm{g} / \mathrm{ml}$ of gentamicin. To knockout HIF1A and EPAS1 by CRISPR/Cas9 editing, gRNA targeting exon 1 of HIF1A or EPAS1 was individually cloned into the pX462-puromycin and pX462-hygromycin vectors (expressing Cas9n, AddGene), respectively. MDA-MB-231 cells were transfected with pX462-puro-HIF1A gRNAs using FuGene HD followed by selection in puromycin. A clonal line that had no HIF1A protein detected by immunoblotting was transfected with pX462-hygromycin-EPAS1 gRNA and selected by hygromycin. Hygromycinresistant colonies that had no EPAS1 detected by immunoblotting were pooled to generate a HIF1A/EPAS1 double knockout (HIF-dKO) subline. For all KD or KO sublines, control cells were transfected with corresponding empty vectors (EV) and selected in antibiotics in parallel with cells transfected with shRNAs or gRNAs. Pooled drug-resistant colonies of control cells were used as EV control lines.

\section{Orthotropic xenograft and experimental lung metastasis models}

All in vivo studies were performed in accordance with the protocols approved by the Institutional Animal Care and Use Committee (IACUC) of the University of Tennessee Health Science Center. NOD. Cg PrkdcscidI12rgtm1Wj1/Szj (NSG) mice were purchased from Jackson Laboratories (Bar Harbor, ME, USA). Orthotropic xenograft and experimental lung metastasis (tail-vein injection) models were established by using fluorescence labelled tumor cells, as previously described $[95,139]$. Tumor size was monitored and measured weekly using digital calipers. Tumor volume was calculated as: volume $=\left(\right.$ width $^{2} \mathrm{x}$ length $) / 2$. Lung metastasis was quantified by fluorescent imaging of lungs and qPCR of human Alu DNA repeats (Forward primer: 5':

GTCAGGAGATCGAGACCATCCC 3', Reverse primer: 5': 
TCCTGCCTCAGCCTCCCAAG 3'). Circulating tumor cells in whole blood (collected by cardiac puncture) were isolated by using the Ficoll-Paque PLUS medium (GE Healthcare Life Sciences, Piscataway, NJ, USA) and counted under fluorescent microscope.

\section{Migration, invasion and wound healing assays}

Transwell membrane inserts with 8-micron pores (BD Biosciences, Bedford, MA, USA), uncoated or coated with Matrigel, were used to determine the migratory and invasive activities of cancer cells, respectively. Cells undergoing migration and invasion were expressed as: Percent migration $=$ mean number of cells migrating through the uncoated transwell x $100 /$ mean number of seeded cells. Percent invasion $=$ mean number of cells migrating through the matrigel-coated transwell x 100 / mean number of migrating cells through the uncoated pores). Real-time assessment of migratory activity during scratch-wound healing was performed by using the IncuCyte ZOOM-ImageLock plate system (Essen Bioscience, Michigan, USA). To examine the expression levels of proteins in response to scratch wounds, the EMD Millipore Chemicon Cell Comb Scratch assay kit (Millipore) was used to generate a high-density field of scratches in a confluent cell monolayer to maximize the area of wound edges. To examine the effect of HBEGF on cell migration and invasion, a neutralizing antibody of HBEGF $(10 \mu \mathrm{g} / \mathrm{ml}$, AF-259NA, R\&D systems, Minneapolis, MN, USA) or a HBEGF peptide $(20 \mu \mathrm{g} / \mathrm{ml})$ was added to the medium.

\section{Suspension culture, viable cell counting and caspase assays}

To mimic the loss of attachment, cells were cultured in PolyHEMA (Sigma Aldrich) coated plates to prevent adherence. Methylcellulose (1\%) was added into medium to prevent formation of large cell aggregates to accurately measure tumor cell proliferation in suspension. Viable cells were counted by Trypan Blue exclusion method. Cell apoptosis was determined by using the caspase Glo 3/7 assay kit (Promega, Madison, Wi, USA) or immunoblotting of cleaved Caspase 9.

\section{Luciferase reporter assay for HIF activity}

Luciferase reporter constructs driven by hypoxia responsive elements of LDHA (LDHA-Luc, S721613) and ITGA6 (ITGA6-Luc, S708174) were purchased from

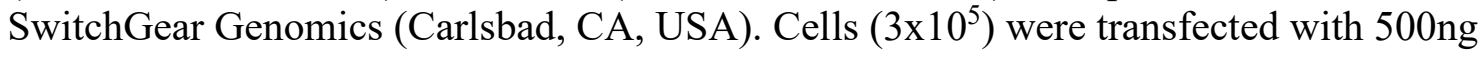
LDHA- or ITGA6-Luc and 10 ng CMV- $\beta$-Galactosidase (control for transfection efficiency), along with 250ng of an empty vector or a HIF1A-expressing construct (HsCD00444875, DNASU plasmid repository), by using Fugene6 (Promega). Forty-eight hours after transfection the cells were exposed to hypoxia $(1 \% \mathrm{O} 2)$ for six hours.

Luciferase and $\beta$-gal activities were measured by using the LightSwitch Luciferase Assay 
System and Promega Beta-Glo Assay System, respectively. Relative luciferase activities normalized to $\beta$-gal were presented as: mean $\pm S D, n=6$.

\section{Exosome isolation and analysis}

To isolate exosomes, $5 \times 10^{6}$ cells were cultured in medium supplemented with exosome-free serum (SystemBio, Palo Alto, CA, USA). Exosomes in the conditioned medium were purified by using the ExoQuick-TC solution (SystemBio), and quantified under a fluorescent microscope after labeled with carboxyfluorescein diacetate succinimidyl ester (CFSE) by using the Exo-Glow labeling kit (SystemBio) that is designed to exclude background particles. To analyze the protein content, isolated exosomes were lysed in RIPA buffer (Pierce, ThermoScientific) containing protease inhibitor cocktail (SigmaAldrich) and subjected to immunoblot analysis. To examine the effect of purified exosomes on cell migration, exosomes were re-suspended in exosomefree medium and added to cells seeded in Transwells to evaluate migration.

\section{Gene expression microarray and $q P C R$ analysis}

Total RNA from cells exposed to hypoxia $\left(1 \% \mathrm{O}_{2}\right.$ for 6 and $\left.48 \mathrm{~h}\right)$ was purified by using the RNeasy kits (Qiagen) and submitted to the Molecular Resource Center at University of Tennessee Health Science Center for labelling and hybridization to the HT12 expression BeadChips (Illumina, Chicago, IL, USA). Hybridization signals were processed for annotation, background subtraction, quantile normalization and presence call filtering by using the Gene Expression Module of the GenomeStudio Software (Illumina). The microarray data can be found in the Gene Expression Omnibus database with accession number of GSE107300 (secure token: wbafiwcgptkvjwf). Hypoxia responsive genes were defined as genes whose expression was altered by $\geq 1.5$ fold (hypoxia vs. control) in two independent experiments. BHLHE40 target genes were defined as genes whose expression was altered by $\geq 1.5$ fold (BHLHE40-KD vs. EV) in two independent experiments. To examine the effect of BHLHE40-KD on gene expression in cells exposed to hypoxia $\left(1 \% \mathrm{O}_{2}\right)$ in combination with low glucose $(1 \mathrm{mM})$ $\left(1 \% \mathrm{O}_{2} / \mathrm{LG}\right)$, a condition frequently encountered by cells in solid tumors, total RNAs of cells exposed to $\left(1 \% \mathrm{O}_{2} / \mathrm{LG}\right.$ for $4 \mathrm{~h}$ from three independent experiments were pooled and analyzed using the GeneChip Human Gene 1.0 ST array (Affymetrix, Santa Clara, CA, USA). The Affymetrix data were extracted, normalized, and summarized with the robust multi-average (RMA) method implemented in the Affymetrix Expression Console. To validate microarray data by qPCR, total RNAs from cells in three independent experiments with duplicates was prepared by using trizol (Life Technologies, Grand Island, NY, USA). cDNA's were synthesized using iScript cDNA Synthesis kits (BioRad, Hercules, CA, USA) and qPCR was performed on the CFX96TM Real-Time PCR detection system by using SYBR green supermix (Bio-Rad). Expression data of mRNA were normalized by the $2-\Delta \Delta C T$ method to RPL13A and presented as mean \pm standard

deviation (SD). Primers of qPCR were obtained from the Primerbank [141]. 


\section{Protein extraction, co-immunoprecipitation and immunoblotting}

For immunoblotting (IB) analysis, whole cell lysates and nuclear proteins were prepared by using RIPA buffer supplemented with protease inhibitor cocktails (SigmaAldrich) and the NE-PER Nuclear and Cytoplasmic Extraction Reagents (Thermo Scientific), respectively. To detect protein-protein interaction, soluble proteins were extracted by using the Pierce IP Lysis Buffer (Thermo Scientific) supplemented with protease inhibitor cocktails and co-immunoprecipitation was performed by using the TrueBlot Immunoprecipitation and Western Blot Kit (Rockland Immunochemicals Inc., Limerick, PA). IB signals were developed by using the SuperSignal West Dura Extended Duration Substrate and the CL-XPosure Film (Thermo Scientific). Antibodies used in this study were: anti-EGFR-Tyr1110P, anti-EGFR, anti-ERK1/2-Thr202/Tyr204P, antiERK1/2, anti-AKT-Tyr416P, anti-AKT, anti-Caspase9, anti-HDAC1 and anti-HDAC2 from Cell Signaling Technologies (Boston, MA, USA); anti-GAPDH from Millipore (Merck, Darmstadt, Germany); anti-TBP from Abcam (Cambridge, MA, USA); antiCTGF from Abgent (San Diego, CA, USA); anti-HBEGF and anti-CD9 from R\&D Biosystems (Minneapolis, MN, USA); anti-BHLHE40, anti-HIF1A and anti-EPAS1 from Novus Biologicals (Littleton, CO, USA); and anti-ALIX, anti-TSG101, anti-CD81 from Santa Cruz (Dallas, TX, USA).

\section{Chromatin immunoprecipitation}

Protein-DNA crosslink, nuclear fraction extraction and chromatin fragmentation were performed as described previously [95]. Soluble fraction of sheared chromatin (200500bp in length) was pre-cleaned with Magnabind Goat anti-Rabbit IgG (for antiBHLHE40 and anti-HDAC1) or Magnabind Goat anti-Mouse (for anti-HDAC2) (Life Technologies), followed by immunoprecipitation with control IgG or antibodies against BHLHE40, HDAC1 or HDAC2, and Magnabind beads conjugated with a secondary antibody. DNA in the decrosslinked immunocomplexes was isolated with the MiniElute PCR purification kit (Qiagen, Germantown, MD, USA). qPCR was performed to detect the presence of proximal promoter region of HBEGF (-529 to -372 from the transcription start site) by using the following primers: forward 5' TGCCTGCAACTTCAACT CTG 3' and reverse 5' CCATCCCTGTCACCCTCTAA 3'.

\section{Statistical analysis}

Student's $t$-tests, one-way ANOVA with post-hoc Tukey test and Pearson $\mathrm{r}$ correlation significance analyses were performed by using the GraphPad Prism 5 software (Graphpad, San Deigo, CA, USA); $p$ values $<0.05(*)$ were considered statistically significant. 


\section{Results}

\section{BHLHE40 knockdown leads to decreased primary tumor growth and lung metastases}

To define the role of BHLHE40 in breast cancer metastasis, we examined the effect of its knockdown (KD) by a shRNA lentiviral construct on spontaneous lung metastasis of orthotopic xenograft tumors derived from a lung metastasis-enriched subline (LM) of breast cancer MDA-MB-231 cells [83]. The protein levels of BHLHE40 is significantly induced by hypoxia $\left(1 \% \mathrm{O}_{2}, 16 \mathrm{~h}\right)$. BHLHE40-shRNA expression effectively reduced both baseline and hypoxia-induced levels of BHLHE40 in LM cells (Figure 3-1A). In NSG mice inoculated with $2 \times 10^{5}$ control LM-EV (empty vector) cells in the inguinal mammary gland fat pads, palpable tumors were detected at 2 weeks (Figure 3-1B) and lung metastasis became evident at 5 weeks (Figure 3-1C) postinoculation. BHLHE40-KD delayed the onset of primary tumors, which became palpable 3 weeks after inoculation, and reduced the growth rate of primary tumors, coincident with decreased lung metastases (Figure 3-1A, B, and C). To further investigate the effect of BHLHE40-KD on lung metastases, primary tumors of EV and BHLHE40-KD cells were surgically removed at 3 and 5 weeks post-inoculation, respectively, when they reached similar size with a diameter of 4-5 mm. Lung metastasis was examined four weeks after primary tumor resection (Figure 3-1D). BHLHE40-KD substantially reduced lung metastasis in mice with similar primary tumor burdens. Taken together, these results suggest that BHLHE40 plays a role in promoting primary tumor growth and spontaneous distant metastasis of breast cancer cells.

\section{BHLHE40 knockdown reduces lung colonization of tumor cells inoculated through tail vein}

To determine whether BHLHE40 regulates late metastatic events after entry of tumor cells into blood stream, we examined the effect of BHLHE40-KD on the ability of tumor cells to survive circulation and colonize in the lungs by using an experimental metastasis model, in which tumor cells were delivered into blood stream through tail-vein injection to bypass the initial steps of metastasis, namely, migration and intravasation.

LM-EV and LM-BHLHE40-KD cells $\left(5 \times 10^{5}\right)$ were injected into the left lateral tail-vein of 5-week-old female NSG mice, and tumor cells in the bloodstream and lung tissues were examined at various time-points post-injection (Figure 3-2). Compared to control LM-EV cells, LM-BHLHE40-KD cells were more rapidly eliminated from the bloodstream (Figure 3-2A). LM-EV cells were observed in lung tissues at 72 hours and formed large metastatic foci at 4 weeks after tail vein injection (Figure 3-2B and $\mathbf{C}$ ). In contrast, BHLHE40-KD cells were not detected in lung tissue at $72 \mathrm{~h}$, and formed lower metastatic foci in lungs than EV cells at various time points (Figure 3-2B and C). No fluorescent loci of EV or BHLHE40-KD cells were found in other organs (i.e., livers, spleens and kidneys) within 5 weeks after tail vein inoculation. Together, these results 

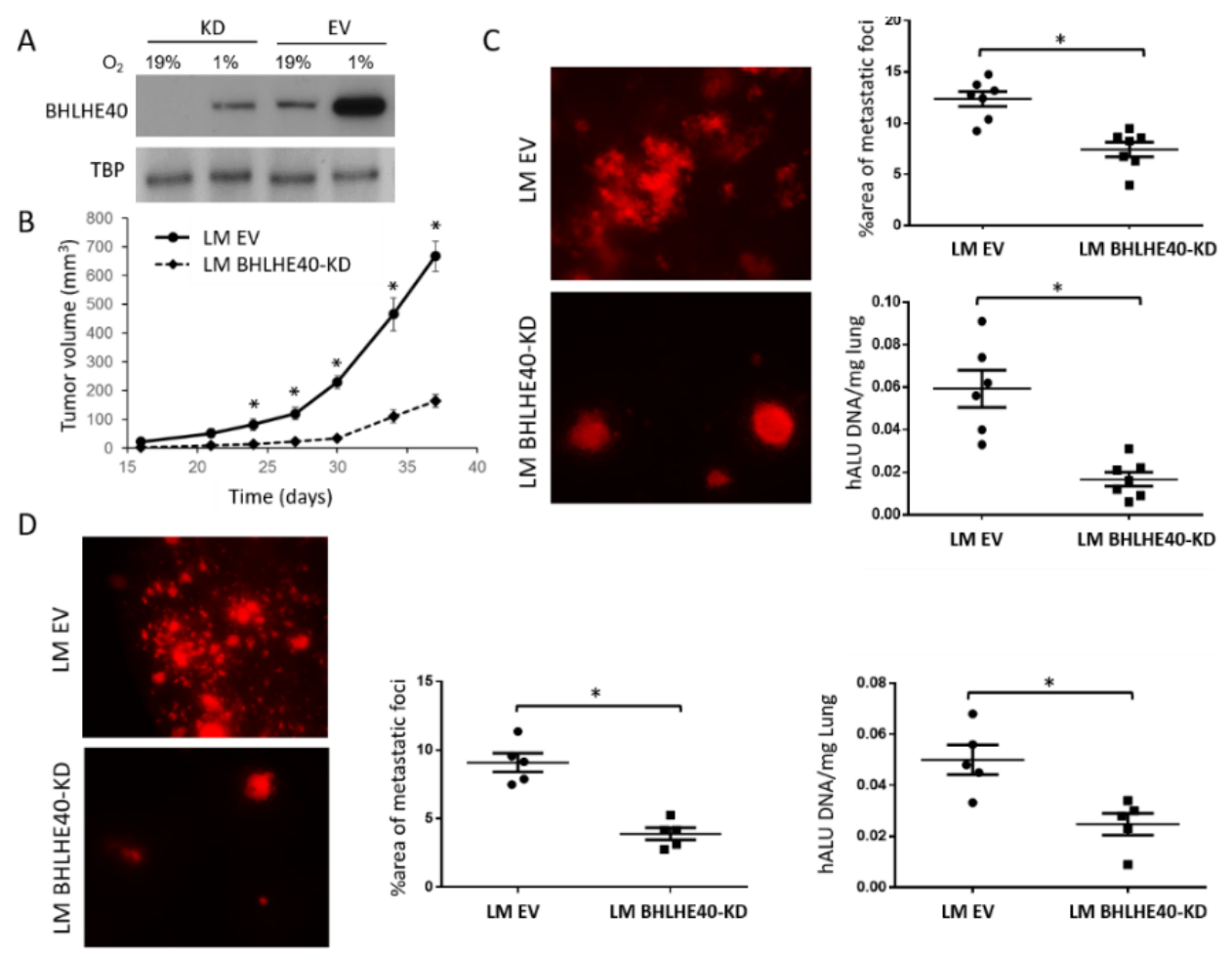

Figure 3-1. BHLHE40 knockdown (KD) significantly reduced primary tumor size and lung metastatic burden in an orthotopic xenograft model.

A. BHLHE40-shRNA expression effectively reduced both baseline and hypoxia-induced expression of BHLHE40 protein in the LM cells, as determined by Immunoblotting. B. Orthotopic xenograft tumors derived from LM-BHLHE40-KD cells exhibited lower growth rate than tumors derived from control LM EV (empty vector) cells. NSG mice were inoculated in the inguinal mammary gland fat pads with $2 \times 10^{5}$ cells. Tumor size was monitored and measured weekly by using a digital caliper. Tumor volume was calculated as: volume $=\left(\right.$ width $^{2} \mathrm{x}$ length $) / 2 .{ }^{*} \mathrm{p}<0.05$ ( $\mathrm{n}=20, \mathrm{KD} v s . \mathrm{EV}$ at indicated time points $)$, One-way ANOVA followed by Tukey's post-hoc tests. C. Spontaneous lung metastasis detected by fluorescent imaging of lungs or human ALU repeats qPCR 5-wk after inoculation of tumor cells in mammary gland fat pads. ${ }^{*} \mathrm{p}<0.05(\mathrm{n}=10, \mathrm{KD} v s . \mathrm{EV})$, student's $t$ test. D. Lung metastasis in mice after resection of primary tumors. Primary tumors in mammary gland fat pads were resected when they reached a size of $5 \times 5 \mathrm{~mm}$ and lung metastasis were analyzed 4 weeks post-resection by fluorescent imaging of lungs or human ALU repeats qPCR. ${ }^{*} p<0.05$, (n=10, KD vs. EV), student's t test. 
A

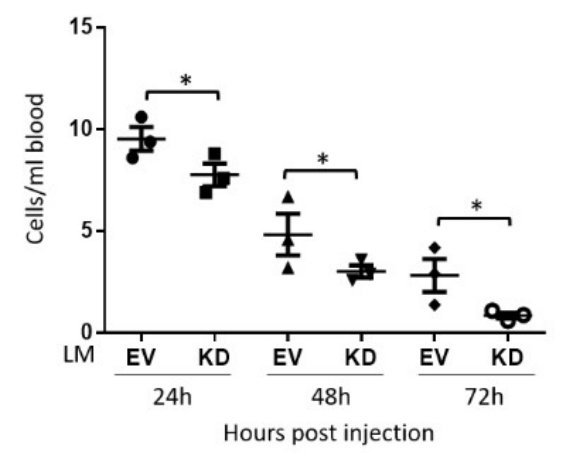

B
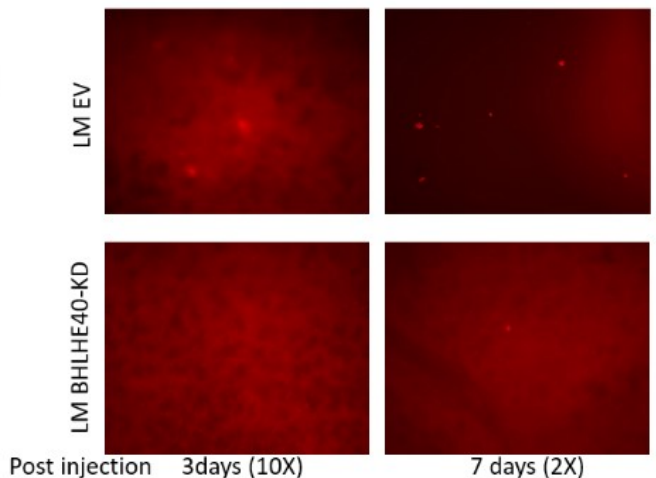

7 days $(2 X)$
C
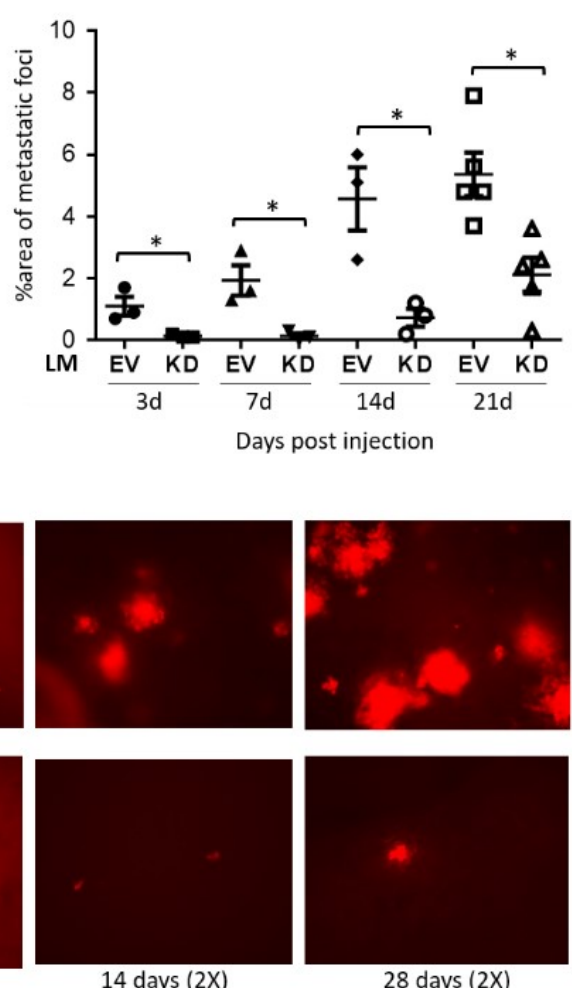

Figure 3-2. BHLHE40 knockdown (KD) reduced lung colonization of tumor cells inoculated into the circulation via tail veins.

A. Less tumor cells were detected in blood of NSG mice inoculated via tail vein injection with LM BHLHE40-KD than mice inoculated with empty vector control LM EV cells. Tumor cells in whole blood collected by cardiac puncture at indicated times after tail vein injection were isolated by using the Ficoll-Paque PLUS medium and counted under fluorescent microscope. ${ }^{*} \mathrm{p}<0.05(\mathrm{n}=3, \mathrm{KD} v s$. EV), One-way ANOVA followed by Tukey's post-hoc tests. B. Fluorescent imaging of metastatic foci in lungs at different time points post tail-vein injection. C. Percentage of areas occupied by metastatic loci in the lungs, as quantified by fluorescent imaging and Image ${ }^{*}{ }^{*}<0.05$, ( $\left.\mathrm{n}=3, \mathrm{KD} v s . \mathrm{EV}\right)$, One-way ANOVA followed by Tukey's post-hoc tests. 
suggest that BHLHE40 is required for tumor cells to survive circulation and establish metastatic foci in the lungs.

\section{BHLHE40 acts to promote cell migration, invasion and survival}

Having established a role of BHLHE40 in distant metastasis of breast cancer cells in vivo, we sought to identify the specific cellular processes that require BHLHE40 activity. Despite the significant effect of BHLHE40-KD on primary tumor growth and lung metastasis of LM cells in vivo, BHLHE40-KD showed no significant effect on proliferation of LM cells under normal 2-D growth conditions in vitro. The doubling times, determined by trypan blue exclusion-based cell counting (daily for 7 days), of LMEV and LM-BHLHE40-KD cells were $36.37 \pm 0.49 \mathrm{~h}(\mathrm{n}=6)$ and $38.95 \pm 3.61 \mathrm{~h}(\mathrm{n}=6)$, respectively. Therefore, we focused on investigating whether BHLHE40 is a downstream effector of HIF1A activation by hypoxia or loss of attachment. Detached breast cells were reported to rely on HIF1A activation to survive under normoxia [142]. In vitro cell migration and invasion assays showed that BHLHE40-KD reduced the ability of cells to penetrate either uncoated or matrigel-coated transwells under hypoxia condition $\left(1 \% \mathrm{O}_{2}\right.$, Figure 3-3A). Under the non-adherent culture condition for 15 days, in which cells were mixed with growth medium supplemented with $1 \%$ methylcellulose to prevent cell aggregation and then seeded in plates coated with polyHEMA to prevent adherence, the number of viable LM BHLHE40-KD cell was significantly lower than LM EV cells (Figure 3-3A, lower panel). To examine whether the effects exerted by BHLHE40-KD on LM cells can be extended to the parent MDA-MB-231 cells, we established a BHLHE40 knockout (KO) subline by using the CRISPR/Cas9 editing system. BHLHE40 protein depletion in the $\mathrm{KO}$ subline under normoxia or hypoxia was confirmed by immunoblotting (Figure 3-3B, upper panel). BHLHE40-KO resulted in reduced number of viable cells after a 15-day suspension culture in plates coated with polyHEMA to prevent attachment (Figure 3-3B, middle panel), while showed no significant effect on number of viable cells after a 5-day adherence culture. In addition, BHLHE40-KO significantly sensitized MDA-MB-231 cells to apoptosis induced by hypoxia in combination with glucose depletion $\left(1 \% \mathrm{O}_{2} / \mathrm{GF}, 6 \mathrm{~h}\right)$, as evidenced by the appearance of apoptotic morphology and activation of caspase3/7 (Figure 3-3B, lower panel).

We further examined the function of BHLHE40 in breast tumor cells with elevated baseline activation of HIF1 A and BHLHE40 by using the tamoxifen-resistant (TR) and fulvestrant-resistant (FR) variants of MCF7 cells [140]. As shown in Figure 33C, mRNA expression levels of BHLHE40 and HIF1A are significantly elevated in TR and FR cells in comparison to parent MCF7 cells. BHLHE40-KD in TR cells substantially increased apoptosis induced by glucose depletion, under both normoxia and hypoxia conditions, as well as reduced number of viable cells after a 15-day suspension culture in polyHEMA-coated plates (Figure 3-3C). Collectively, these observations provide evidence supporting a role of BHLHE40 in promoting survival and migration. 
A
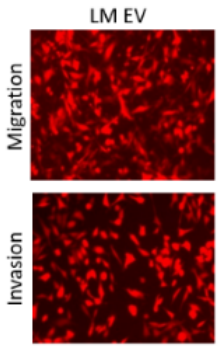

LM EV

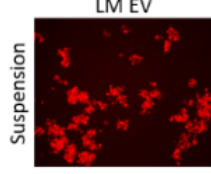

C

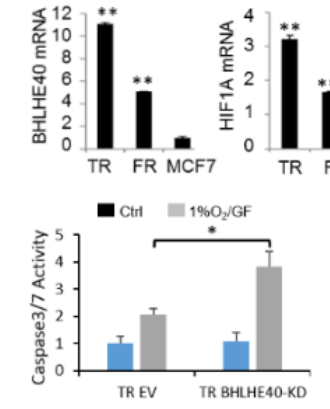

LM BHLHE40-KD
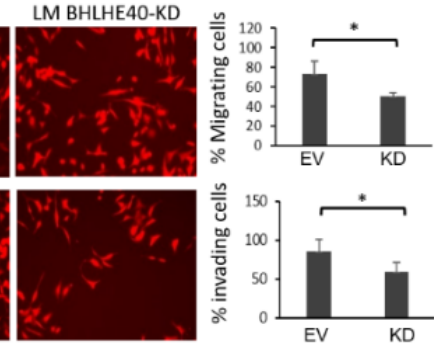

LM BHLHE40-KD
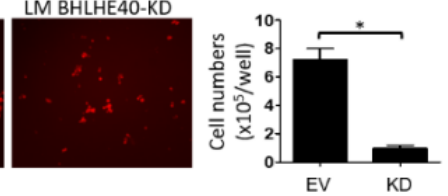

B
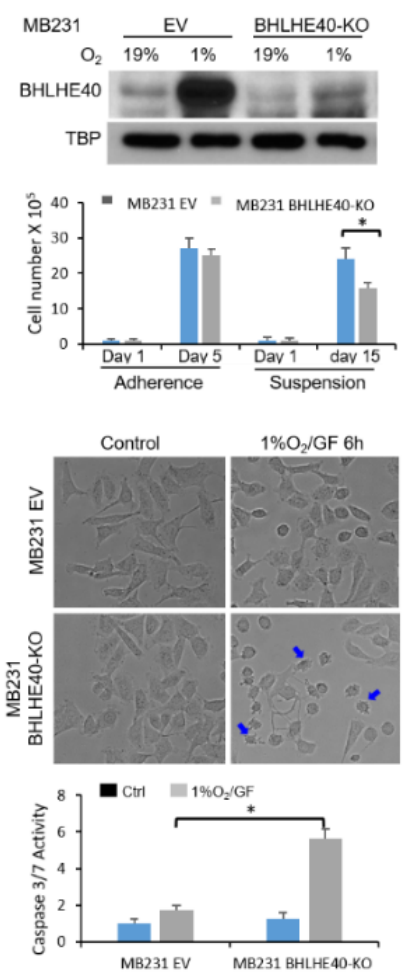

Figure 3-3. BHLHE40 depletion impaired cell migration, invasion and survival.

A. BHLHE40 knockdown (KD) by shRNA in LM cells reduced cell migration and invasion, as well as the number of viable cells during suspension culture in comparison to LM EV cells. Migratory and invasive activities were determined by using Transwells, uncoated or coated with Matrigel, respectively. Suspension culture was conducted by seeding cells in medium containing $1 \%$ Methylcellulose and in dishes coated with PolyHEMA for 15 days. ${ }^{*} p<0.05$ ( $\mathrm{n}=6$, KD $v$ s. EV), student's t test. B. BHLHE40 knockout (KO) by CRISPR/Cas9 editing in MDA-MB-231 cells reduced the number of viable cells after suspension culture and enhanced apoptosis induced by hypoxia combined with glucose depletion $\left(1 \% \mathrm{O}_{2} / \mathrm{GF}\right)$. Viable cells were determined by trypan blue exclusion-based cell counting after a 15-day suspension culture. Apoptosis of cells exposed to $1 \% \mathrm{O}_{2} / \mathrm{GF}$ for $6 \mathrm{~h}$ was examined by the appearance of apoptotic morphology (as indicated by arrows in the cell images) and Caspase $3 / 7$ assays. ${ }^{*} p<0.05$ ( $\mathrm{n}=6, \mathrm{KO} v s$. EV), One-way ANOVA followed by Tukey's post-hoc tests. C. BHLHE40-KD by shRNA in tamoxifen-resistant subline of MCF7 (TR) reduced the ability of cells to survive $1 \% \mathrm{O}_{2} / \mathrm{GF}(6 \mathrm{~h})$ and reduced number of viable cells after a 15 -day suspension culture. Elevated expression of HIF1A and BHLHE40 in TR and Fulvestrant-resistant (FR) sublines of MCF7 cells were detected by qPCR. BHLHE40-KD was confirmed by immunoblotting. Apoptosis induced by $1 \% \mathrm{O}_{2} / \mathrm{GF}(6 \mathrm{~h})$ was determined by Caspase $3 / 7$ assays. Number of viable cells after a 15-day suspension culture was determined by trypan blue exclusion-based cell counting. ${ }^{* *} \mathrm{P}<0.05$ ( $\mathrm{n}=6$, TR or FR vs. parent MCF7), ${ }^{*} \mathrm{p}<0.05(\mathrm{n}=6, \mathrm{KD}$ vs. EV), One-way ANOVA followed by Tukey's post-hoc tests. 


\section{BHLHE40 is required for transcription activation of a set of cytokines and growth factors}

To delineate the molecular pathways regulated by BHLHE40, we performed global gene expression analysis of LM-EV and LM BHLHE40-KD cells exposed to hypoxia $\left(1 \% \mathrm{O}_{2}, 6 \mathrm{~h}\right.$ or $\left.48 \mathrm{~h}\right)$. The microarray data can be found in the Gene Expression Omnibus database with accession number of GSE107300. Overall, the expression levels of 521 and 646 genes in LM-EV cells were altered (fold change $\geq 1.5$ in two independent experiments) by hypoxia at $6 \mathrm{~h}$ and $48 \mathrm{~h}$, respectively. BHLHE40-KD abolished the hypoxia-mediated upregulation of 45 (out of 261, 17.2\%) and 98 (out of 361, 27.1\%) genes at $6 \mathrm{~h}$ and $48 \mathrm{~h}$, respectively. In addition, BHLHE40-KD abolished the hypoxiamediated downregulation of 30 (out of 260,10.5\%) and 44 (out of 285, 15.4\%) genes at $6 \mathrm{~h}$ and $48 \mathrm{~h}$, respectively. The hypoxia-induced genes that were affected by BHLHE40$\mathrm{KD}$ were overrepresented by genes that encode proteins with cytokine or growth factor activities as defined by Gene Ontology annotation (GO:0005125 and GO:0008083, Fisher's exact $\mathrm{p}<0.0001$ ) (Figure 3-4A). The expression of a subset of these genes was also reduced in BHLHE40-KD cells exposed to $1 \% \mathrm{O}_{2} / \mathrm{LG}$ for $4 \mathrm{~h}$, compared to $\mathrm{EV}$ cell (Figure 3-4B). In contrast, hypoxia-induced expression of a panel of the core hypoxia responsive genes that are known to be directly targeted by HIF1A [129] was not significantly affected by BHLHE40-KD (Figure 3-4C). These observations suggest that BHLHE40-KD preferentially reduced hypoxia-induced expression of a set of cytokines and growth factors, but did not cause a global defect in HIF1A-mediated transcription activation. To confirm this notion, we examined the effect of BHLHE40-KO on HIFmediated expression of reporter luciferase driven by well-characterized HIF1A-binding sites in the promoter regions of LDHA and ITGA6 [26-28]. As showed in Figure 3-4D, BHLHE40-KD exhibited no significant effect on hypoxia-induced luciferase activities, in the absence or presence of exogenous HIF1A protein. The effect of BHLHE40-KD on expression of cytokines and growth factors in cells exposed to $1 \% \mathrm{O} 2 / \mathrm{LG}(4 \mathrm{~h})$ was validated by qPCR (Figure 3-4E). Consistent with results in BHLHE40-KD cells cultured in vitro, we detected reduced expression of connective tissue growth factor (CTGF) and HBEGF, at both mRNA and protein levels, in the LM-BHLHE40-KD primary tumors in comparison to LM-EV tumors established in mouse mammary gland fat pads (Figure 3-4F).

To determine whether BHLHE40-mediated expression of genes encoding cytokine or growth factors is relevant to clinical samples, we analyzed the mRNA expression data of breast tumors in the TCGA database [109, 143]. The expression of $71.4 \%$ (20 out of 28) of these BHLHE40-dependent genes (as showed in Figure 3-4A) was found to be positively correlated with BHLHE40 expression with statistical significance $(p<0.05)$ in at least one of the four major subtypes of breast tumors (Table 3-1). This observation provides supporting evidence for a role of BHLHE40 in the expression of these genes in human breast tumors.

Since hypoxia-induced cytokines and growth factors are commonly exported to extracellular space by exosomes [144], we sought to determine whether BHLHE40-KD 

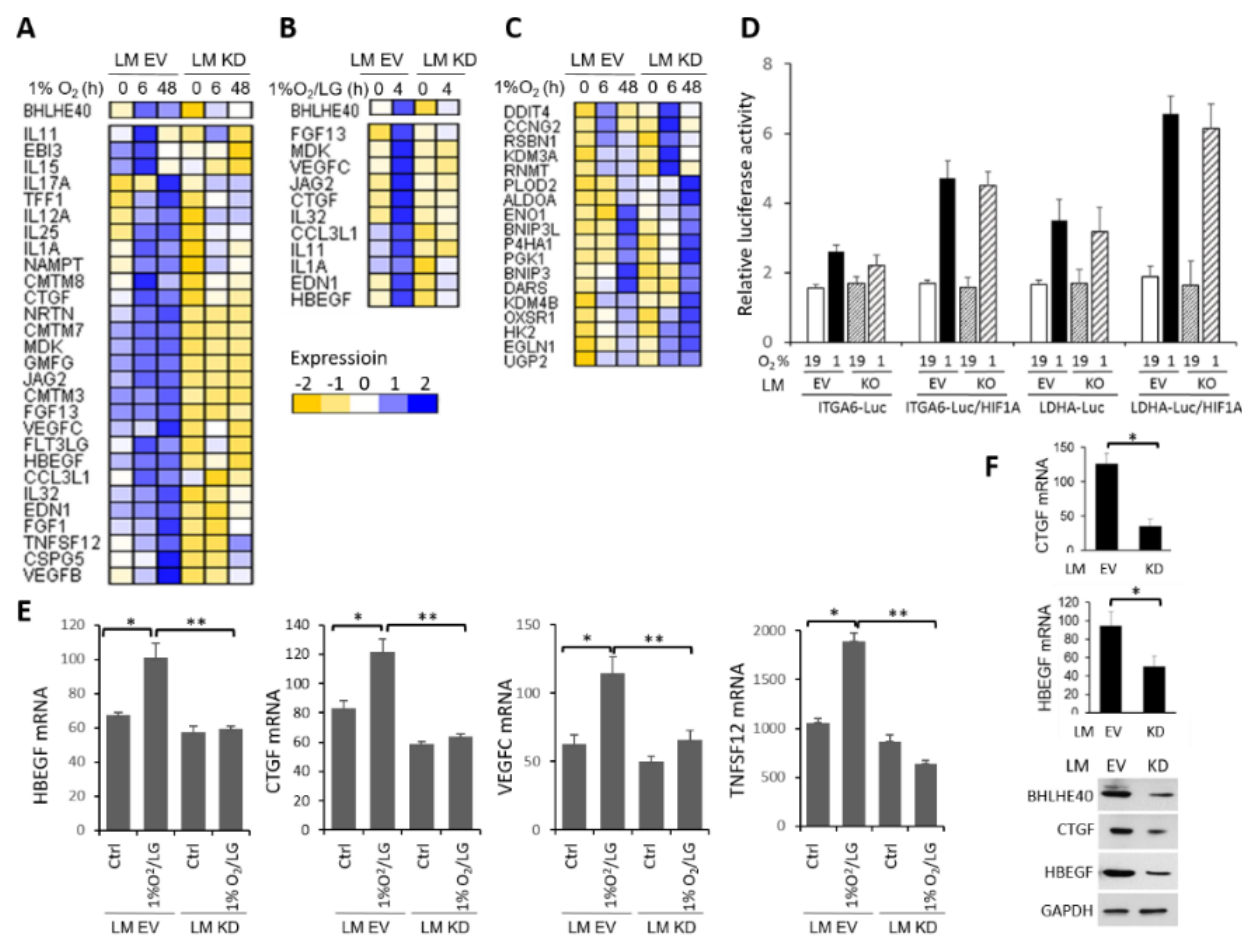

Figure 3-4. BHLHE40 knockdown reduced hypoxia-induced expression of a panel of cytokines and growth factors.

A. Heatmaps of cytokines and growth factors whose hypoxia-induced expression $\left(1 \% \mathrm{O}_{2}\right.$ at $6 \mathrm{~h}$ or $48 \mathrm{~h}$, fold change $\geq 1.5$ in two independent experiments) was diminished by BHLHE40-knockdown (KD) in LM cells. The gene expression levels were determined by using the Illumina Human HT-12 expression BeadChips. Normalized (Quantile normalization) hybridization signals were $\log 2$ transformed and standardized by genes across experiment conditions to generate heatmap. B. Heatmaps of a subset of genes list in A whose expression was affected by BHLHE40-KD in LM cells exposed to hypoxia combined with low $(1 \mathrm{mM})$ glucose $\left(1 \% \mathrm{O}_{2} / \mathrm{LG}, 4 \mathrm{~h}\right)$. The gene expression levels were determined by using the Affymetrix Human Gene 1.0 ST array. C. Heatmaps of hypoxiainduced genes whose expression was not significantly affected by BHLHE40-KD in LM cells as determined the Illumina Human HT-12 expression BeadChips. D. Expression of luciferase reporters driven by hypoxia-responsive elements of ITGA6 or LDHA was not affected by BHLHE40 knockout (KO) by CRISPR/Cas9 editing in MDA-MB-231 cells, in the absence or presence of exogenous HIF1A. Luciferase activities were normalized to co-transfected CMV- $\beta$-galactosidase and presented as mean $\pm \mathrm{SD}(\mathrm{n}=6)$. E. Expression of genes in control LM EV and LM BHLHE40-KD cells exposed to $1 \% \mathrm{O}_{2} / \mathrm{LG}(4 \mathrm{~h})$. mRNA expression levels were determined by qPCR, normalized to RPL13A, and presented as mean $\pm \mathrm{SD}(\mathrm{n}=6) .{ }^{*} p<0.05\left(\mathrm{n}=6,1 \% \mathrm{O}_{2} / \mathrm{LG} v s\right.$. untreated control), ${ }^{*} p<0.05(\mathrm{n}=6, \mathrm{KD} v s$. EV), One-way ANOVA followed by Tukey's post-hoc tests. F. mRNA and protein expression levels of HBEGF and CTGF in primary xenograft tumors, determined by qPCR and immunoblotting, respectively. ${ }^{*} p<0.05$ ( $\left.\mathrm{n}=6, \mathrm{KD} v s . \mathrm{EV}\right)$, Student's t test. Representative Immunoblotting images of 3 tumors of KD or EV cells were presented. 
Table 3-1. Correlated expression of BHLHE40 and its putative targets in breast tumors (TCGA).

\begin{tabular}{|c|c|c|c|c|c|c|c|c|}
\hline \multirow[b]{2}{*}{ Gene } & \multicolumn{2}{|c|}{ BL $(n=230)$} & \multicolumn{2}{|c|}{ Her2 $(n=162)$} & \multicolumn{2}{|c|}{ LA $(n=315)$} & \multicolumn{2}{|c|}{ LB $(n=300)$} \\
\hline & $\begin{array}{c}\text { Pearson } \\
\text { r value }\end{array}$ & P value & $\begin{array}{c}\text { Pearson } \\
\text { r value }\end{array}$ & $P$ value & $\begin{array}{c}\text { Pearson } \\
\text { r value }\end{array}$ & P value & $\begin{array}{c}\text { Pearson } \\
\text { r value }\end{array}$ & $P$ value \\
\hline CCL3L1 & 0.1210 & 0.0694 & -0.2045 & 0.0095 & 0.0504 & 0.3792 & 0.0480 & 0.4136 \\
\hline CMTM3 & -0.0042 & 0.9494 & 0.0358 & 0.6510 & 0.5065 & $<0.0001$ & -0.0521 & 0.3687 \\
\hline СMTM7 & -0.2761 & $<0.0001$ & -0.0697 & 0.3780 & 0.2516 & $<0.0001$ & 0.0635 & 0.2729 \\
\hline СМТМ8 & -0.1580 & 0.0165 & -0.0886 & 0.2624 & 0.1034 & 0.0670 & -0.0013 & 0.9817 \\
\hline CSPG5 & -0.1333 & 0.0435 & -0.0309 & 0.6961 & -0.0351 & 0.5345 & -0.0504 & 0.3850 \\
\hline CTGF & 0.0203 & 0.7593 & 0.3087 & $<0.0001$ & 0.4876 & $<0.0001$ & 0.0631 & 0.2760 \\
\hline EBI3 & 0.2135 & 0.0011 & -0.2060 & 0.0085 & 0.0805 & 0.1543 & -0.0271 & 0.6402 \\
\hline EDN1 & 0.0705 & 0.2867 & 0.0928 & 0.2401 & 0.3805 & $<0.0001$ & 0.0273 & 0.6379 \\
\hline FGF1 & 0.1666 & 0.0114 & 0.2470 & 0.0015 & 0.4225 & $<0.0001$ & 0.0357 & 0.5384 \\
\hline FGF13 & -0.1105 & 0.0946 & -0.1782 & 0.0233 & 0.1393 & 0.0134 & -0.1276 & 0.0271 \\
\hline FLT3LG & 0.2436 & 0.0002 & -0.0588 & 0.4573 & 0.2878 & $<0.0001$ & -0.0264 & 0.6487 \\
\hline GMFG & 0.2593 & $<0.0001$ & -0.1764 & 0.0247 & 0.3367 & $<0.0001$ & -0.1271 & 0.0277 \\
\hline HBEGF & 0.3871 & $<0.0001$ & 0.2873 & 0.0002 & 0.3254 & $<0.0001$ & 0.1577 & 0.0062 \\
\hline IL11 & 0.1413 & 0.0322 & 0.1762 & 0.0249 & 0.2053 & 0.0002 & 0.0328 & 0.5710 \\
\hline IL12A & 0.0325 & 0.6207 & -0.0582 & 0.4618 & -0.1035 & 0.0649 & -0.1543 & 0.0069 \\
\hline IL15 & 0.3407 & $<0.0001$ & 0.0628 & 0.4273 & 0.1632 & 0.0037 & -0.0913 & 0.1146 \\
\hline IL17A & 0.1036 & 0.1139 & 0.0845 & 0.2850 & -0.0221 & 0.6942 & -0.0919 & 0.1094 \\
\hline IL1A & 0.2140 & 0.0013 & -0.0057 & 0.9440 & 0.0399 & 0.4960 & -0.0720 & 0.2359 \\
\hline IL25 & -0.1011 & 0.3633 & 0.0560 & 0.6578 & -0.1569 & 0.0293 & 0.1617 & 0.0661 \\
\hline IL32 & 0.1597 & 0.0153 & -0.0571 & 0.4704 & 0.3242 & $<0.0001$ & -0.0473 & 0.4141 \\
\hline JAG2 & -0.0418 & 0.5287 & 0.0387 & 0.6250 & 0.0880 & 0.1190 & -0.0167 & 0.7729 \\
\hline MDK & -0.0837 & 0.2057 & -0.0947 & 0.2305 & 0.1107 & 0.0496 & 0.0066 & 0.9099 \\
\hline NAMPT & 0.2678 & $<0.0001$ & 0.0873 & 0.2691 & 0.1667 & 0.0030 & -0.1819 & 0.0016 \\
\hline NRTN & -0.4457 & $<0.0001$ & -0.0350 & 0.6596 & 0.0672 & 0.2411 & -0.0288 & 0.6220 \\
\hline TFF1 & 0.2156 & 0.0009 & -0.0148 & 0.8516 & 0.0221 & 0.6936 & -0.1243 & 0.0300 \\
\hline TNFSF12 & 0.1690 & 0.0102 & -0.0271 & 0.7323 & 0.1482 & 0.0084 & 0.0565 & 0.3296 \\
\hline VEGFB & -0.0460 & 0.4879 & 0.0811 & 0.3049 & 0.2028 & 0.0003 & 0.0443 & 0.4449 \\
\hline VEGFC & 0.3664 & $<0.0001$ & 0.1760 & 0.0251 & 1.0000 & $<0.0001$ & -0.0922 & 0.1110 \\
\hline
\end{tabular}

HBEGF expression is significantly correlated with BHLHE40 in basal-like, Her2positive, Luminal A, and Luminal B subtypes of breast cancer. The correlation analysis was performed using the mRNA expression Z-scores (RNA Seq V2 RSEM, TCGA). BL, basal-like; Her2, ERBB2-enriched; LA, Luminal A; LB, Luminal B. (Highlighted cells show significant positive correlation) 
could affect exosome secretion. As shown in Figure 3-5, the numbers of isolated exosomes were significantly reduced in the conditioned medium from MDA-MB-231 BHLHE40-KO and TR-BHLHE40-KD cells in comparison to the corresponding control cells cultured under both normal condition or exposed to $1 \% \mathrm{O}_{2} / \mathrm{LG}$ for $6 \mathrm{~h}$. The presence of exosomic markers (i.e., CD9, CD81, ALIX and TSG101) [145, 146]) in the isolated exosomes was confirmed by immunoblotting (Figure 3-5). BHLHE40 depletion reduced protein levels of HBEGF in the purified exosomes (Figure 3-5), reflecting the reduced levels of HBEGF mRNA and HBEGF protein in whole cell extracts of BHLHE40-KD or KO cells (Figure 3-4E and Figure 3-5). These observations suggest that BHLHE40depletion reduced exosome secretion because of reduced expression of cytokines and growth factors.

\section{BHLHE40 activates HBEGF transcription by sequestering HDAC1 and HDAC2 from promoter binding}

Among the cytokines and growth factors affected by BHLHE40-KD in LM cells, the expression level of HBEGF mRNA is positively correlated with the expression level BHLHE40 mRNA in all four major subtypes of breast tumors in the TCGA database (Figure 3-4, Figure 3-5 and Table 3-1). HBEGF is a heparin-binding EGF-like growth factor that promotes cell proliferation and invasion through EGFR activation [147]. To examine the molecular mechanism underlying BHLHE40-mediated HBEGF transcription, we performed ChIP analysis. BHLHE40 binding to the proximal promoter region of HBEGF was not affected by $1 \% \mathrm{O} 2 / \mathrm{LG}$ (data not shown), indicating that HBEGF transcription activation was not caused by increased BHLHE40-DNA binding. However, $1 \% \mathrm{O} 2 / \mathrm{LG}$ treatment reduced binding of HDAC1 and HDAC2 to the HBEGF promoter (Figure 3-6A), which is coincident with increased BHLHE40-HDAC1/2 interaction in the soluble cellular fraction, as detected by reciprocal Coimmunoprecipitation (CoIP) followed by immunoblotting (IB) (Figure 3-6B). In cells lacking BHLHE40, HDAC1/HDAC2 remained bound to the promoter region of HBEGF after 1\%O2/LG treatment (Figure 3-6A). This result suggests that BHLHE40 plays a role in facilitating dissociation of $\mathrm{HDAC} 1 / 2$ from promoters through protein-protein interaction. To examine whether HDAC1/2-DNA binding plays a key role to suppress transcription of BHLHE40 target genes, we examine the effect of HDAC inhibitors on mRNA expression of HBEGF, CTGF and VEGFC. As shown in Figure 3-6C, both HDAC2-specific (BRD6688, $10 \mu \mathrm{M}$ ) and pan-HDAC inhibitor (TSA, $2 \mu \mathrm{M}$ ) increased expression of BHLHE40 target genes in MDA-MB-231 EV and BHLHE40-KD cells, supporting a role of $\mathrm{HDAC} 1 / 2$ in suppressing transcription of BHLHE40 target genes. Taken together, these observations suggest that sequestering HDAC1/2 from DNA binding contributes to BHLHE40-mediated transcription activation.

\section{HBEGF acts to promote cell survival and migration}

To examine whether BHLHE40-driven HBEGF expression plays a role in EGFR

activation to promote cell survival, we examined the phosphorylation status of EGFR and 
A

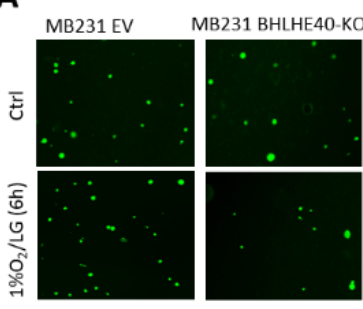

= MB231 EV $=$ MB231 BHLHE40-KO

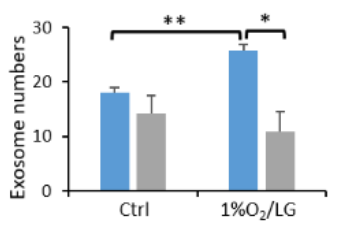

MB231 EV KO EV KO
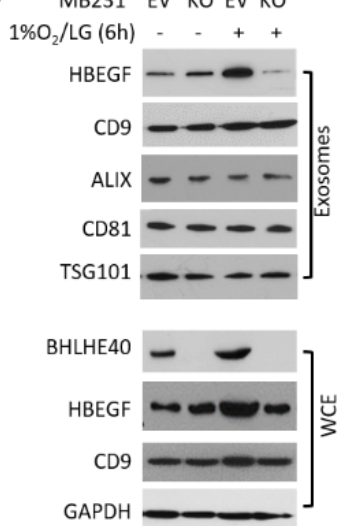

B

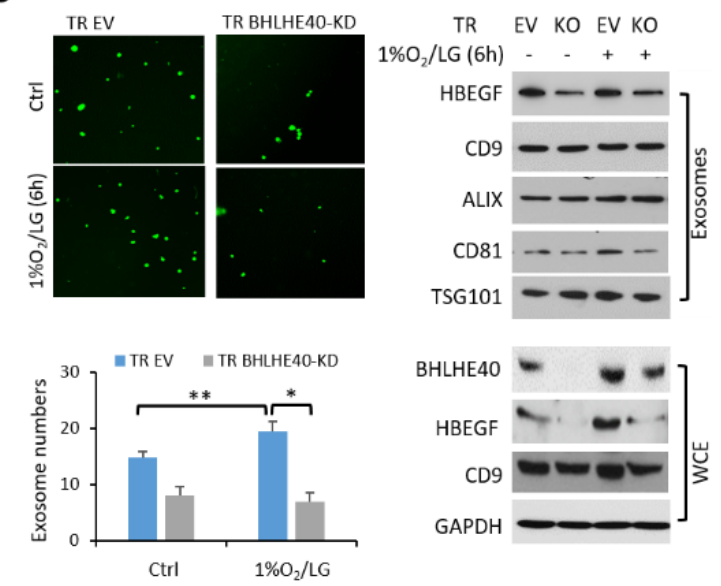

Figure 3-5. Exosomic secretion of HBEGF was reduced by BHLHE40 depletion in MDA-MB231 or TR cells.

A. BHLHE40-KO by CRISPR/Cas9 editing in MDA-MB-231 cells reduced the total number of exosomes and the amount of HBEGF protein in exosomes in comparison to empty vector control EV cells. B. BHLHE40 knockdown (KD) by shRNA in TR cells reduced the total number of exosomes and the amount of HBEGF protein in exosomes in comparison to TR EV cells. Exosomes were purified from conditioned medium of $5 \times 10^{6}$ cells cultured in medium supplemented with exosome-free serum, either under normal culture condition or exposed to $1 \% \mathrm{O} 2 / \mathrm{LG}$ for $6 \mathrm{~h}$. Exosomes were purified by using the ExoQuick-TC solution and quantified under a fluorescent microscope after labeled with carboxyfluorescein diacetate succinimidyl ester (CFSE) by using the Exo-Glow labeling kit, which is designed to exclude background particles. Exosome number in the bar graph is presented as mean number of exosomes per field $\pm \mathrm{SD}$ (total 9 fields from three independent experiments were examined). The presence of HBEGF and exosome markers in purified exosomes ( $3 \mu \mathrm{g}$ protein/lane) or whole cell extracts (WCE, $30 \mu \mathrm{g}$ protein/lane) were detected by immunoblotting. $* * \mathrm{P}<0.05\left(\mathrm{n}=9,1 \% \mathrm{O}_{2} / \mathrm{LG} v s\right.$. control), $* p<0.05(\mathrm{n}=9, \mathrm{KO}$ or KD $v s$. EV), One-way ANOVA followed by Tukey's post-hoc tests. 
A
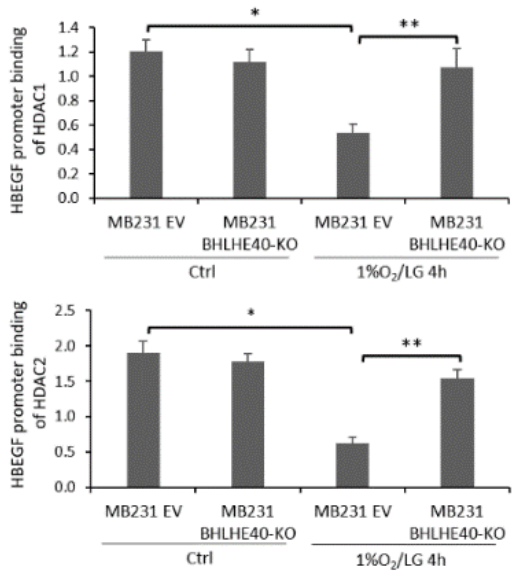

B

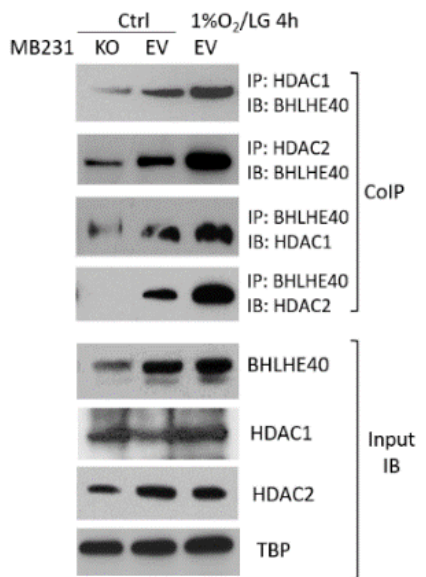

C
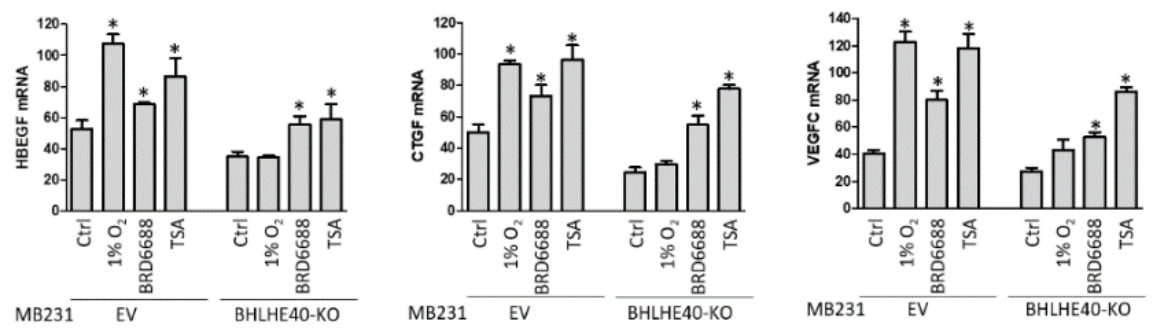

Figure 3-6. BHLHE40 activates gene expression by sequestering HDAC1 and HDAC2 from genome DNA binding in MDA-MB-231 cells exposed to hypoxia and low glucose $\left(1 \% \mathrm{O}_{2} / \mathrm{LG}, 4 \mathrm{~h}\right)$.

A. BHLHE40-knockout (KO) diminished dissociation of HDAC1 and HDAC2 from the promoter region of $\mathrm{HBEGF}$ in MDA-MB-231 cells exposed to $1 \% \mathrm{O}_{2} / \mathrm{LG}(4 \mathrm{~h})$, as determined by chromatin immunoprecipitation (ChIP) followed by qPCR of the HBEGF promoter region ( -529 to -372 from the transcription start site). HBEGF promoter binding activity of HDAC1 or HDAC2 was calculated as: [(DNA amount in anti-HADC IP complex - DNA amount in control IgG IP complex)/DNA amount in $1 \%$ input]. ${ }^{*} \mathrm{p}<0.05$ $\left(\mathrm{n}=6,1 \% \mathrm{O}_{2} / \mathrm{LG} v s\right.$. control), ${ }^{*} \mathrm{p}<0.05(\mathrm{n}=6, \mathrm{KO} v s . \mathrm{EV})$. B. $1 \% \mathrm{O}_{2} / \mathrm{LG}$ treatment increased interactions between BHLHE40 and HDAC1/2 in the soluble cellular fraction of MDA-MB-231 EV cells. Protein-protein interaction was detected by reciprocal coimmunoprecipitation (IP)/immunoblotting (IB) analysis. C. HDAC inhibition induced expression of BHLHE40 target genes. Cells were exposed to hypoxia $\left(1 \% \mathrm{O}_{2}\right)$ or HDAC inhibitors (BRD6688 $10 \mu \mathrm{M}$ or TSA $2 \mu \mathrm{M}$ ) for $24 \mathrm{~h}$. mRNA expression levels were determined by qPCR, normalized to RPL13A, and presented as mean $\pm \mathrm{SD}(\mathrm{n}=6) .{ }^{*} p$ $<0.05$ ( $\mathrm{n}=6$, treated $v s$. untreated control cells), One-way ANOVA followed by Tukey's post-hoc tests. 
its downstream targets in MDA-MB-231 and TR sublines exposed to 1\%O2/GF for $6 \mathrm{~h}$, a condition known to induce apoptosis as showed in Figure 3-3. Compared to cells with intact BHLHE40 activity, MDA-MB-231-BHLHE40-KO and TR-BHLHE40-KD cells expressed lower levels of HBEGF mRNA and protein, which was coincident with reduced levels of phosphorylation of EGFR, AKT and ERK and increased caspase 9 cleavage (Figure 3-7A and B). Next, we examined whether active HBEGF peptide could rescue MDA-MB-231-BHLHE40-KO cells from apoptosis. As shown in Figure 3-7C, addition of HBEGF peptide into culture medium of cells exposed to $1 \% \mathrm{O} 2 / \mathrm{GF}$ significantly reduced activation of caspase $3 / 7$. These observations provide evidence supporting a role of HBEGF in promoting cell survival.

Monolayer scratch was found to induce expression of HIF1A, BHLHE40 and HBEGF in MDA-MB-231-EV cells (Figure 3-8A), implicating a role of the HIF1ABHLHE40-HBEGF axis in cell migration during wound healing. By using the IncuCyte ZOOM-ImageLock plate system, we demonstrated that BHLHE40-KO substantially diminished the ability of MDA-MB-231 cells to close the wound gaps, which was restored by addition of HBEGF peptide (Figure 3-8B and $\mathbf{C}$ ). In contrast, a HBEGF neutralizing antibody [148] inhibited wound healing of LM-EV cells (Figure 3-8B and C). To confirm that exosomic HBEGF plays a key role in promoting cell migration, we examined the migratory activities of MDA-MB-231 BHLHE40-KO cells in the presence of conditioned medium or purified exosomes, which were collected from the MDA-MB$231 \mathrm{EV}$ cells at $24 \mathrm{~h}$ after extensive wound scratch. The Transwell migration assay showed that both conditioned medium and purified exosomes from the wounded EV cells increased the migratory activity of BHLHE40-KO cells (Figure 3-8D). Together, these observations suggest that HBEGF act downstream of BHLHE40 to promote cell migration.

To confirm that BHLHE40 and HBEGF are key downstream effectors of HIFs in promoting cell migration, we examined the effect of BHLHE40 overexpression on a HIF1A/EPAS1 double knockout subline (MDA-MB-231 HIF-dKO). Although HIF1A mRNA expression level is approximately 6-fold higher than EPAS1 mRNA in MDAMB-231 cells according to reported RNAseq data (GSE73526), compensatory activation of EPAS1 could compromise the effect of HIF1A knockout. Therefore, we used HIF$\mathrm{dKO}$ cells to examine whether BHLHE40 overexpression can rescue molecular and phenotypic changes caused by HIF-KO. Gene expression analysis by qPCR showed that $\mathrm{HIF}-\mathrm{dKO}$ reduced baseline and $1 \% \mathrm{O}_{2} / \mathrm{LG}$ induced expression of BHLHE40, HBEGF, CTGF and VEGFC mRNAs, which was restored by BHLHE40 overexpression (Figure 3-9A). In addition, BHLHE40 overexpression reduced cell-cell contact, as showed by cell imaging, and increased migratory activity of HIF-dKO cells, as determined by transwell assays (Figure 3-9B). Immunoblotting analysis confirmed that BHLHE40 overexpression restored expression levels of HBEGF protein in HIF-dKO cells exposed to $1 \% \mathrm{O} 2 / \mathrm{LG}$ (Figure 3-9C). Together, these observations support the notion that BHLHE40 and HBEGF act as key downstream effectors of HIFs to promote cell migration. 

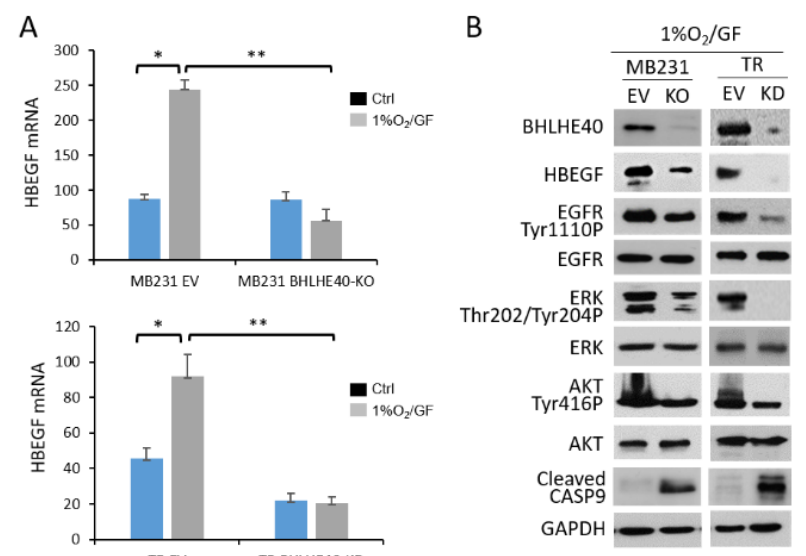

C

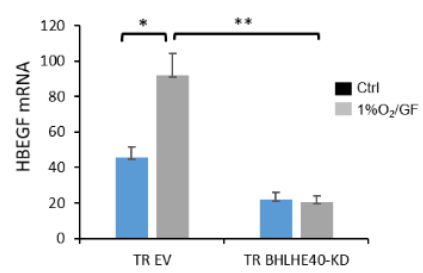

Figure 3-7. BHLHE40 depletion reduced phosphorylation of EGFR, while increased Caspase 9 cleavage, in cells exposed to glucose depletion and hypoxia $\left(1 \% \mathrm{O}_{2} / \mathrm{GF}\right)$.

A. BHLHE40 knockout (KO) by CRISPR/Cas9 editing in MDA-MB-231 and BHLHE40 knockdown (KD) by shRNA in TR cells diminished HBEGF induction by $1 \% \mathrm{O}_{2} / \mathrm{GF}$ (6h). mRNA expression levels were determined by qPCR, normalized to RPL13A, and presented as mean $\pm \mathrm{SD}(\mathrm{n}=6) .{ }^{*} \mathrm{p}<0.05\left(\mathrm{n}=6,1 \% \mathrm{O}_{2} / \mathrm{GF} v s\right.$. control), ${ }^{*} \mathrm{p}<0.05(\mathrm{n}=6, \mathrm{KO}$ $v s$. EV), One-way ANOVA followed by Tukey's post-hoc tests. B. BHLHE40 depletion reduced EGFR activation, as indicated by reduced phosphorylation of EGFR and its downstream targets (ERK and AKT), while increasing apoptosis, as indicated by detection of cleaved caspase 9. Data from three independent immunoblotting analyses are presented. C. HBEGF peptide $(10 \mu \mathrm{g} / \mathrm{ml})$ reduced apoptosis induced by $1 \% \mathrm{O}_{2} / \mathrm{GF}(6 \mathrm{~h})$ in MDA-MB-231 BHLHE40-KO cells. Apoptosis was determined by Caspase 3/7 assays. ${ }^{*} \mathrm{p}<0.05\left(\mathrm{n}=6,1 \% \mathrm{O}_{2} / \mathrm{GF}\right.$ vs. control), ${ }^{*} \mathrm{p}<0.05(\mathrm{n}=6$, HBEGF $v s$. untreated with HBEGF), One-way ANOVA followed by Tukey's post-hoc tests. 
A

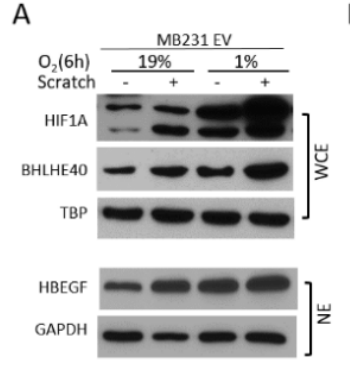

B

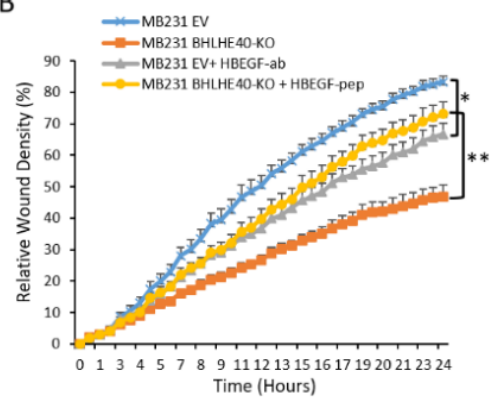

C
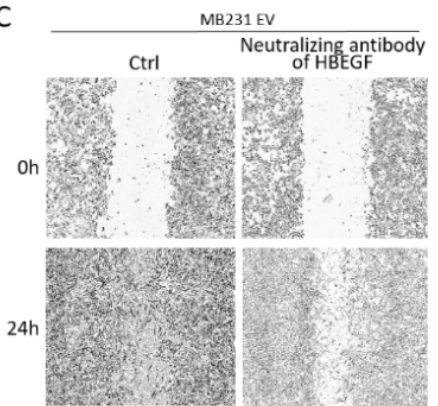

MB231 BHLHE4OKO

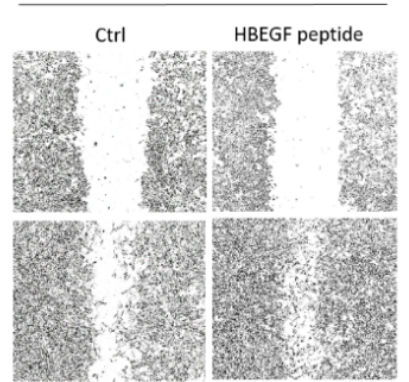

D
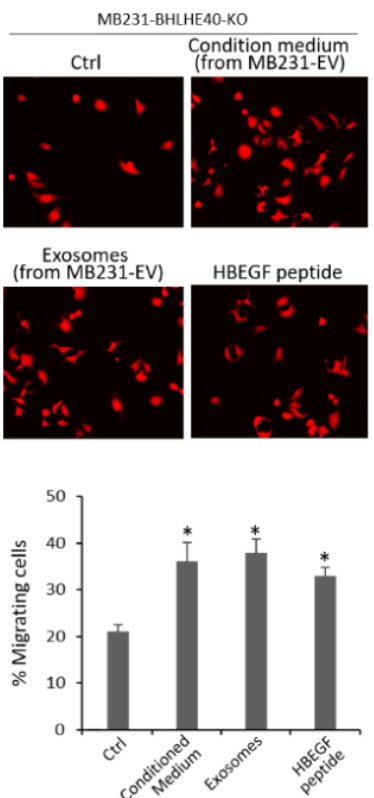

Figure 3-8. HIF-BHLHE40-HBEGF axis plays a role in promoting cell migration during wound healing.

A. Monolayer scratch increased protein levels of HIF1A, BHLHE40 and HBEGF in MDA-MB-231 EV cells. Intensive scratch wounds were generated by using the EMD Millipore's Cell Comb scratch assay kit and immunoblotting was performed $6 \mathrm{~h}$ after cells cultured under normoxia $\left(19 \% \mathrm{O}_{2}\right)$ or hypoxia $\left(1 \% \mathrm{O}_{2}\right)$. B. BHLHE40 knockout $(\mathrm{KO})$ by CRISPR/Cas9 editing reduced the migratory activity of MDA-MB-231 cells, which was restored by addition of HBEGF peptide into the culture medium. In contrast, a HBEGF neutralizing antibody reduced the migratory activity of MDA-MB-231-EV cells. Realtime assessment of migratory activity after wound scratch was performed by using the IncuCyte ZOOM-ImageLock plate system. ${ }^{*} \mathrm{P}<0.05(\mathrm{n}=6$, time points $6-24 \mathrm{~h}$, HBEGF $v s$. untreated), ${ }^{*} \mathrm{p}<0.05(\mathrm{n}=6$, time points $6-24 \mathrm{~h}$, anti-HBEGF $v s$. untreated), One-way ANOVA followed by Tukey's post-hoc tests. Representative data from two independent experiments with 6 replicates are presented. C. Images of representative wound fields at 0 and $24 \mathrm{~h}$ after wound scratch as described in B. D. Conditioned medium or purified exosomes from MDA-MB-231-EV cells (24 h after wound scratch) increased migratory activities of MDA-MB-231 BHLHE40-KO cells, as determined by the transwells migration assays. The migrated cells in six fields were imaged and counted under fluorescent microscope. The results were presented as: Percent migration $=$ mean number of cells migrating through the uncoated transwells x 100/mean number of seeded cells; ${ }^{*} \mathrm{p}<0.05(\mathrm{n}=12, v s$. untreated control), One-way ANOVA followed by Tukey's post-hoc tests. 

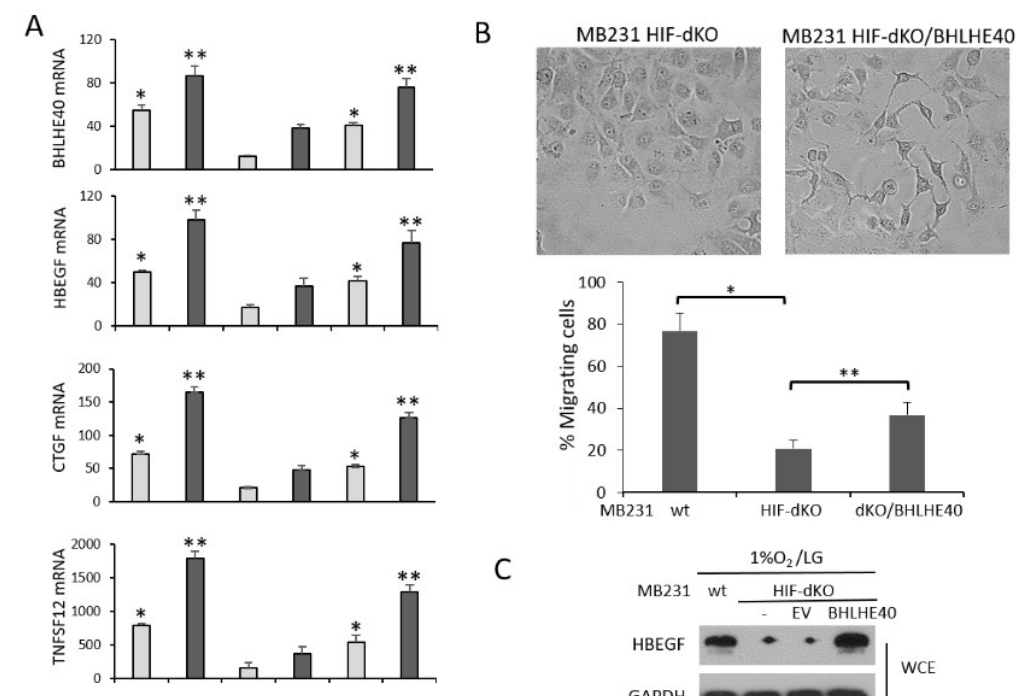

C

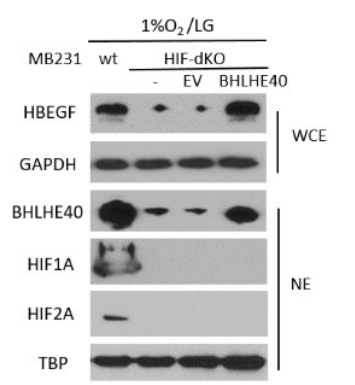

Figure 3-9. Effect of BHLHE40 overexpression on molecular and phenotypic changes caused by HIF1A/EPAS1 double knockout (HIF-dKO) in MDA-MB-231 cells.

A. BHLHE40 overexpression restored baseline and hypoxia/low glucose $\left(1 \% \mathrm{O}_{2} / \mathrm{LG}(6 \mathrm{~h})\right.$ induced expression of HBEGF, CTGF, TNFSF12 and VEGFC in in HIF-dKO cells. The expression levels of mRNAs were determined by qPCR, normalized to RPL13A and presented as mean $\pm \mathrm{SD}(\mathrm{n}=6) .{ }^{*} \mathrm{p}<0.05\left(\mathrm{n}=6, v s\right.$. untreated HIF-dKO), ${ }^{* *} \mathrm{p}<0.05(\mathrm{n}=6, v s$. HIF-dKO exposed to $1 \% \mathrm{O}_{2} / \mathrm{LG}$ for $6 \mathrm{~h}$ ), One-way ANOVA followed by Tukey's posthoc tests B. BHLHE40 overexpression decreased cell-cell contact (as showed by the cell images) and increased migratory activity of HIF-dKO cells exposed to 1\% O2/LG (24h). Migratory activity was determined by Transwell assays and presented as meant of percentage of migrating cells $\pm \mathrm{SD}(\mathrm{n}=6)$. ${ }^{*} \mathrm{p}<0.05(\mathrm{n}=6, \mathrm{HIF}-\mathrm{dKO}$ vs. control wild-type cells), ${ }^{* *} \mathrm{p}<0.05(\mathrm{n}=6$, HIF-dKO/BHLHE40 overexpression $v s$. HIF-dKO), One-way ANOVA followed by Tukey's post-hoc tests. C. BHLHE40 overexpression restored expression of HBEGF proteins in HIF-dKO cells exposed to $1 \% \mathrm{O}_{2} / \mathrm{GF}$ (6h). Proteins were detected by immunoblotting. GAPDH and TBP were used as loading control for whole cell extract (WCE) or Nuclear extract (NE), respectively. 


\section{High expression of BHLHE40 and HBEGF is associated with poor prognosis of breast cancer}

Having established a role of the BHLHE40-HBEGF axis in enhancing cell survival and migration, we sought to examine the association of BHLHE40 and HBEGF with clinical characteristics of breast tumors by using the gene expression data in the Kaplan Meier plotter database, which contains the Affymetrix microarray expression data of 2,178 breast cancer patients [108]. We found that high expression of BHLHE40 or HBEGF is significantly associated with shorter interval of relapse free survival (RFS) among patients diagnosed with triple-negative breast cancer $(\mathrm{TNBC}, \mathrm{n}=255)$ and patients treated with chemotherapy $(n=602)$ (Figure 3-10). However, BHLHE40 and HBEGF are not poor prognostic markers for patients with estrogen receptor positive tumors or patients treated with endocrine therapy. In addition, we analyzed the association of BHLHE40 and HBEGF with overall survival (OS) of TNBC by using the METABRIC dataset in the cBioPortal for Cancer Genomics (Breast Cancer, METABRIC). High BHEGF expression was found to be associated with short interval of OS (Figure 3-10C). Although TNBC with higher expression of BHLHE40 tends to have a shorter interval of OS, this correlation did not reach statistical significance (Figure 3-10C). These findings suggest that activation of the BHLHE40-HBEGF pathway contributes to aggressive behaviors of TNBC and chemoresistance.

\section{Discussion}

Breast cancer metastasis is the major cause of fatality in breast cancer patients. Adaptation to hypoxia is a driving force of metastatic progression and drug resistance [120]. Proteins secreted by tumor cells under hypoxia promote metastasis by altering tumor cell behaviors and modifying the tumor microenvironment [119]. Therefore, the regulation of hypoxia-driven protein secretion is currently under intense investigation. This study, reports a novel role of BHLHE40, a transcription factor directly targeted by HIF1A, in regulating exosomic release of HBEGF. Our results suggest that the HIFBHLHE40-HBEGF axis constitutes an important signaling mechanism to promote metastasis of breast tumors.

Exosomes are 40-100 $\mathrm{nm}$ vesicles that originate from the endocytic compartment. Exosomes contain a wide range of proteins, lipids, mRNAs and microRNAs (miRNAs) that reflect the molecular contents of the parental cells [144]. Compared to normal cells, cancer cells exhibit higher activity of exosome secretion, which is further augmented by stress conditions, including TP53 activation, alteration of intracellular calcium levels, senescence, hypoxia and acidosis [149]. Exosomes released by tumor cells have been reported to contain cytokines and growth factors that promote metastasis and chemoresistance [149-151]. However, the precise molecular mechanism governing the release of exosomes remains elusive. This study however, suggests that BHLHE40 acts as a key downstream effector of HIFs to activate transcription and subsequent exosome secretion of a set of cytokines and growth factors. 
A
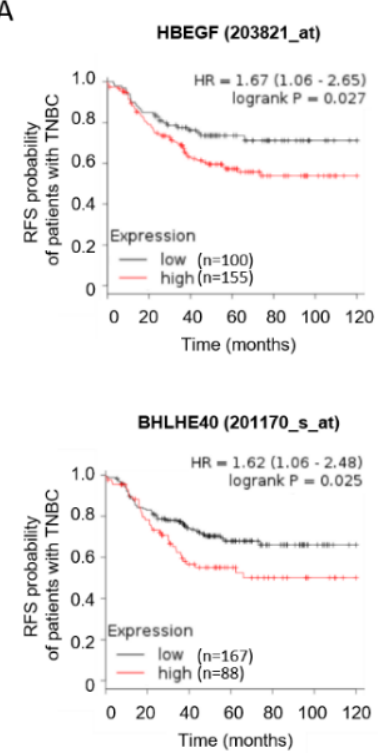

B
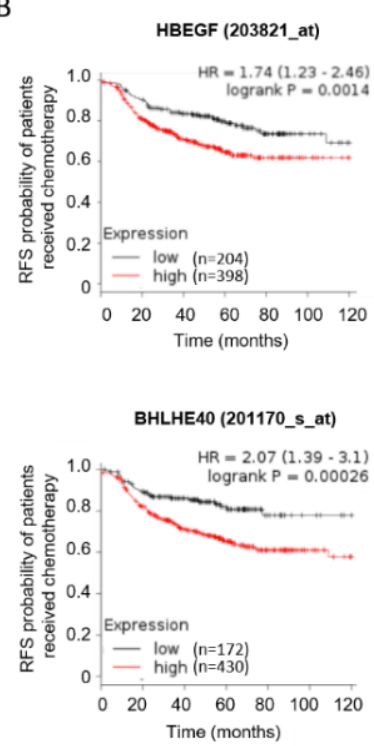

C
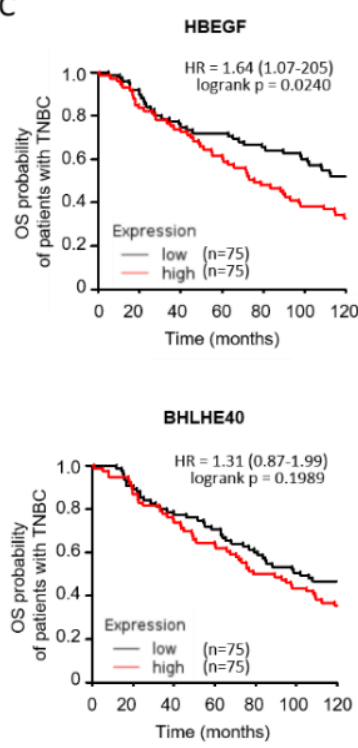

Figure 3-10. High expression of BHLHE40 and HBEGF is associated with poor prognosis of breast cancer.

A. High expression of BHLHE40 and HBEGF is associated with short interval of relapse free survival (RFS) of patients diagnosed with triple-negative breast cancer (TNBC, $\mathrm{n}=255$ ). The gene expression data and patient information were obtained from the Kaplan-Meier plotter database. B. High expression of BHLHE40 and HBEGF is associated with short interval of RFS among patients treated with chemotherapy $(n=602)$. The gene expression data and patient information were obtained from the Kaplan-Meier plotter database. C. High expression of HBEGF is associated with short interval of overall survival (OS) of patients diagnosed with TNBC $(n=150)$. The gene expression data and patient information were obtained from the cBioPortal for Cancer Genomics (Breast Cancer, METABRIC, Nature 2012 \& Nat Commun 2016). 
BHLHE40 was previously described as a transcriptional repressor that binds to the class B E-box (CACGTG) and recruits HDAC1 and HDAC2 to block transcription [152]. BHLHE40 activation has been linked to cell cycle arrest, senescence, differentiation and apoptosis [153-155]. On the other hand, emerging evidence supports a role of BHLHE40 in transcription activation and promoting cell survival. For instance, BHLHE40 was reported to activate transcription of pro-survival factors in tumor cells, including BIRC5 and DeltaNp63 [156, 157]. In addition, BHLHE40 was reported to activate the transcription of a panel of cytokines required for activation of murine CD4+ $\mathrm{T}$ cells $[117,158]$. It remains undefined what factors determine the selectivity of BHLHE40 to suppress or activate transcription. The BHLHE40-mediated transcription activation of DeltaNp63 was shown to depend on its direct interaction with HDAC2 [156]. In agreement with this observation, our results suggest that BHLHE40 activates HBEGF transcription by sequestering $\mathrm{HDAC} 1 / 2$ from DNA binding. It remains to be determined whether interfering with HDAC1/2-DNA binding is a general mechanism responsible for BHLHE40-mediated transcription activation.

Elevated EGFR activation is known to promote survival, proliferation and invasion of tumor cells under hypoxia, and multiple mechanisms have been linked to hypoxia-induced EGFR activation [159]. For example, EPAS1 activation by hypoxia was shown to increase EGFR mRNA translation [160]. Hypoxia-mediated activation of metalloproteases (e.g., ADAM12 and ADAM17) was reported to activate EGFR by increasing ectodomain shedding of HBEGF $[161,162]$. Our study provides a novel aspect of EGFR activation through the BHLHE40-HBEGF axis. In addition to autocrine or paracrine effects within tumor cells, exosomic release of HBEGF might exert paracrine effect to remodel tumor stroma or endocrine effect to prime distant metastatic niches [163].

In conclusion, this study provides evidence supporting an essential role of BHLHE40 in exosomic release of HBEGF, a critical pro-survival and pro-metastasis factor. The clinical relevance of our findings is evidenced by the fact that the elevated expression of BHLHE40 and HBEGF in breast tumors is associated with poor prognosis of patients with TNBC and chemoresistance. Therapeutic intervention targeting the BHLHE40-HBEGF axis may represent an effective approach to combat hypoxia-driven drug resistance and metastasis. 


\section{CHAPTER 4. SUMMARY}

When mammalian cells encounter hypoxia, they develop a variety of mechanisms to cope with this stress and survive, as summarized in Table 4-1 [164]. Approximately 1$1.5 \%$ of the genome is transcriptionally responsive to hypoxia. However, there is significant heterogeneity in the transcriptional response to hypoxia between different cell types [165]. For instance, in the context of tumor cells, HIF1A can impart protumorigenic abilities by direct upregulation of targets like VEGF, LOX and MMP's while in the context of innate immunity, HIF1A can regulate T cells and lymphocytes to promote inflammation by regulating the secretion of cytokines such as CCL5 and IL6 [166]. Therefore, strategies exploiting hypoxic response for therapy need to take into consideration the cell context. This study identifies two HIF1A mediated pathways that require the presence of other transcription regulators (i.e., SMARCE1 and BHLHE40) to impart pro-metastatic abilities to breast cancer cells.

Low oxygen concentration of solid tumors has been linked to increased migratory and invasive potential [19] a pre-requisite to metastatic breast cancer. HIF1A, master controller of hypoxia, acts via multiple pathways to bestow on cancer cells pro-survival and pro-metastatic abilities [167]. To develop strategies to prevent HIF1A-driven tumor progression requires a detailed understanding of upstream regulators and downstream effectors of HIF1A signaling pathways. Our results discover two downstream pathways, HIF1A/SMARCE1-PTK2 and HIF1A-BHLHE40-HBEGF, that HIF1A exploits to impart an increased metastatic potential to the breast cancer cells. Future studies are warranted to evaluate the efficacy of strategies targeting HIF1A-SMARCE1 interaction, PTK2 activation and HBEGF secretion.

\section{Key-findings}

Key findings of this study are listed below (and represented in Figure 4-1).

- Knockdown of EHMT2, SMARCA4, and KDM6B did not affect the metastatic potential of MDA-MB-231 cells.

- SMARCE1 knockdown does not affect the primary tumor growth but significantly reduced spontaneous lung metastases in an orthotopic xenograft model of breast cancer

- SMARCE1 is required for HIF1A-mediated transactivation of PTK2 in response to cell detachment. HIF1A/PTK2 pathway plays a key role in protecting circulating tumor cells against anoikis.

- BHLHE40 knockdown reduced both primary tumor burden and spontaneous lung metastasis in-vivo 
Table 4-1. Summary of epigenetic factors involved in hypoxia-regulated gene expression.

\begin{tabular}{|c|c|c|c|}
\hline Category & $\begin{array}{l}\text { Chromatin } \\
\text { modifiers } \\
\text { involved }\end{array}$ & $\begin{array}{l}\text { Genes or proteins } \\
\text { regulated }\end{array}$ & Phenotypic changes \\
\hline $\begin{array}{l}\text { Transcriptional co- } \\
\text { regulators }\end{array}$ & $\begin{array}{l}\text { CITED1, } \\
\text { HDAC, PCAF, } \\
\text { PPARGC1A, } \\
\text { PKM2, MCM, } \\
\text { FHL }\end{array}$ & $\begin{array}{l}\text { Various hypoxia- } \\
\text { responsive genes, TP53, } \\
\text { HIF1A, BID, VEGF, } \\
\text { LDHA, PDK1, } \\
\text { SLC2A1, HK1 }\end{array}$ & $\begin{array}{l}\text { Angiogenesis, cell } \\
\text { cycle progression, } \\
\text { glycolytic pathway, } \\
\text { cell proliferation, and } \\
\text { other processes } \\
\text { regulated by hypoxia }\end{array}$ \\
\hline $\begin{array}{l}\text { Chromatin- } \\
\text { modifying } \\
\text { complexes }\end{array}$ & $\begin{array}{l}\text { SWI/SNF, } \\
\text { SMARCA4, } \\
\text { ISWI, MTA1 }\end{array}$ & $\begin{array}{l}\text { HIF1A, SHBG, ITIH, } \\
\text { HIF1AN, BNIP3, CA9, } \\
\text { VEGF }\end{array}$ & $\begin{array}{l}\text { Cell cycle arrest, } \\
\text { hematopoietic and } \\
\text { vascular development, } \\
\text { apoptosis, angiogenesis }\end{array}$ \\
\hline $\begin{array}{l}\text { Chromatin } \\
\text { modifiers } \\
\text { mediating hypoxia- } \\
\text { induced EMT }\end{array}$ & $\begin{array}{l}\text { HDAC3, } \\
\text { WDR5 }\end{array}$ & $\begin{array}{l}\text { EMT marker genes } \\
\text { (CDH1, JUP, CDH2, } \\
\text { VIM, etc.) }\end{array}$ & $\begin{array}{l}\text { Migration, invasion, } \\
\text { metastasis }\end{array}$ \\
\hline
\end{tabular}

Hypoxia activated transcriptional co-regulators, chromatin modifying complexes, histone modification enzymes and their role in tumor progression. Reprinted with permission from Kou-Juey Wu, Epigenetic Regulation of hypoxia responsive gene expression: Focusing on chromatin and DNA modifications. International Journal of Cancer, 2013, Volume 134, Issue2. 
A

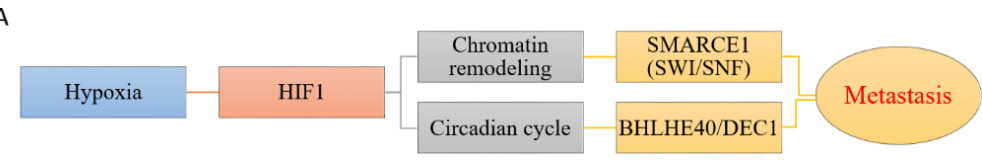

B

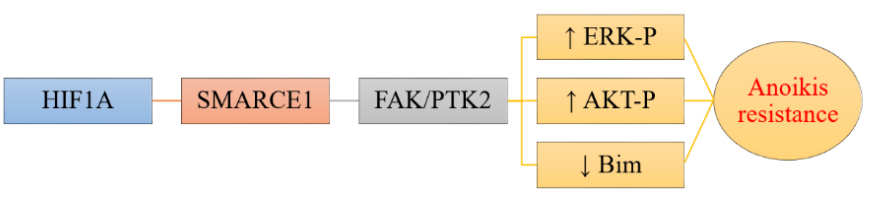

C

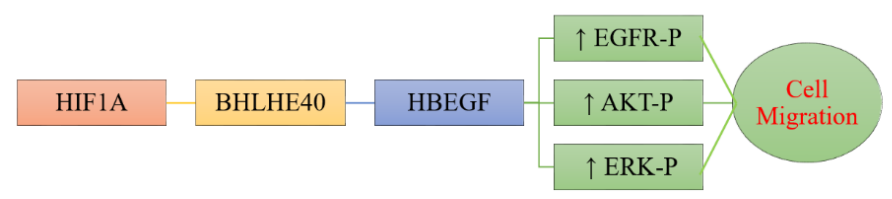

Figure 4-1. Summary of results.

A. HIF1A modulates chromatin remodeling via the SMARCE1 subunit of the SWI/SNF complex and the circadian cycle by direct transcriptional regulation of BHLHE40 leading to tumor progression and metastasis. B. SMARCE1 regulates metastatic potential of breast cancer cells by promoting survival of detached cells through the HIF1A/PTK2 pathway. C. HIF1A-BHLHE40 driven HBEGF activation potentates breast cancer cell survival, invasion and metastasis. 
- BHLHE40 functions as key downstream effector of HIF1A to regulate exosomal secretion of HBEGF, which plays a key role in supporting cell migration and survival.

\section{SMARCE1: Implications in Other Cancers and Further Direction}

Chromatin remodelers have been shown to play a role in tumor progression [167]. One of these important chromatin remodelers is the SWI/SNF nucleosome remodeling complex also implicated in co-activation of HIF1 A target genes [48]. SMARCE1/BAF57 is one of the subunits of this complex. Germline mutation in SMARCE1 predispose young patients to both spinal and intracranial clear cell meningiomas (CCM) and studies reveal heterozygous loss of function mutation in SMARCE1 to be causative of this tumor [168]. This study warrants genetic sequencing of future cases of childhood CCM including investigation of SMARCE1 gene [169] and such mutation screening can help facilitate meningioma classification [170]. SMARCE1 also plays a role in regulating drug response and cytokine secretion of ovarian cancer cells. siRNA mediated knockdown of SMARCE1 was reported to increase cell cycle arrest in G1 when cells were treated with anticancer drugs such as cisplatin, doxorubicin, paclitaxel and 5-fluorouracil [171]. Overexpression of SMARCE1 in SKOV3 ovarian cancer cells resulted in secretion of IL8, MIP1B and RANTES chemokines, which are associated with CD8+ cytotoxic T-cell infiltration. In addition, SMARCE1 has been shown to be a novel target to abrogate androgen receptor-mediated gene activation in prostate cancer [172] [173].

Our study reveals a novel link of SMARCE1 to HIF1A-mediated breast cancer metastasis. PTK2, a non-receptor tyrosine kinase is activated by integrin engagement and growth factor stimulation. Following phosphorylation, PTK2 activates MAPK and AKT pro-survival pathways [174]. PTK2 gene amplification has been reported in various cancers and has been shown to aid in cancer initiation and cell survival. Recently, PTK2 activation has been shown to be required for Src-regulated CDH1 expression in colon cancer cells and inhibition of PTK2 activity reduced cell invasion in these cells. In addition, PTK2 overexpression can modulate ITGB1 to enhance EMT, invasion, and metastasis of cancer cells to the lung [175]. Blocking PTK2 activation has been proven to impair the migratory potential of breast cancer cells [176]. In this study, we identified a novel mechanism of activation of PTK2 by HIF1 A/SMARCE1 in breast cancer cell, which contributes to HIF1A-mediated anoikis resistance.

Further studies are needed to identify other crucial SMARCE1 targets to facilitate the development of SMARCE1-targeted therapy. Additional research should focus on identifying other SWI/SNF accessory subunits involved in the hypoxic response and delineate which domains of SMARCE1 are involved in HIF1A interaction. 


\section{BHLHE40: Implications in Other Cancers and Further Direction}

Deregulation of BHLHE40/DEC1 has been implicated in various types of malignant carcinomas [177]. In human glioblastomas, BHLHE40 facilitates the metabolic adaptability of cells to hypoxia stress by increasing glycolysis [178]. BHLHE40 expression is a poor prognostic marker in pancreatic cancer and modulates chemo sensitivity to gemcitabine [179]. BHLHE40 was reported to be activated by TGFB in colorectal cancer cells to invoke anti-apoptotic response by blocking the apoptotic pathways initiated by mitochondria [180] [181]. Dominant negative mutants of BHLHE40 were shown to prevent lung and liver metastasis of mammary carcinoma cell lines JygMC(A) and 4T1 [182]. In pancreatic cancer, BHLHE40 activation by TGFB has been shown to regulate EMT by regulating the expression of SMAD3, SNAIL, Caludin4, and N-cadherin [133]. Studies in human endometrial cancer cells, however, show that BHLHE40 and BHLHE41 act to block EMT by suppressing TWIST1 expression through competing with SP1 binding to DNA [183]. In addition, BHLHE40 has also been implicated in regulation of cytokine secretion [71], and it plays a key role in production of GM-CSF and IL-10 in T-cells [184]. Our results show that BHLHE40 is required for exosomal secretion of HBEGF in response to HIF1A activation in breast cancer cells. HBEGF, a ligand of EGFR, which plays a key role in promoting breast cancer cell survival and migration [185].

Epidermal growth factor receptor belongs to a family of receptor tyrosine kinase that is anchored in the cytoplasmic membrane and is activated by several ligands like epidermal growth factor (EGF), TGFB, amphiregulin, and HBEGF. Upon activation, EGFR causes activation of the MAPK/ERK, AKT/PI3K, JNK, STAT's and E2F family of proteins that impart pro-survival capabilities to tumor cells. EGFR/MAPK/AKT pathways have been often dysregulated in human cancers [186]. EGFR is overexpressed in 50\% of triple negative breast cancers (TNBC's) and EGFR targeted therapies are being used to enhance the initial sensitivity of TNBC cells to cytotoxic therapy [187]. Cotreatment of EGFR pathway inhibitors with PI3K/AKT pathway inhibitors enhanced the anti-proliferative effects of EGFR inhibitors in MDA-MB-468 cells. Combinational therapy of gefitinib and PI-103 reduced phosphorylation of AKT and MAPK and induced caspase-dependent apoptosis in basal like breast cancer cells [188]. Recent studies have also shown that EGFR signaling can enhance tumor invasion by upregulating MMP-9 expression and PTK2 phosphorylation in MDA-MB-231 and MCF-7 cells [189]. These observations show the importance of EGFR activation in several steps of the metastatic cascade [190-192].

While this study has produced some valuable insights into the role of BHLHE40 in cytokine release induced by HIF1A activation, there are two unanswered questions that need to be addressed. First, the molecular mechanism underlying BHLHE40-mediated transcription activation of HBEGF. Second, whether BHLHE40 plays a role in regulating exosome assembly, transport and cargo loading. Probing for answers to these two questions will facilitate investigation in the therapeutic potential of BHLHE40/HBEGF pathway. 


\section{LIST OF REFERENCES}

1. Foundation; T.S.G.K.B.C., UNDERSTANDING BREAST CANCER SURVIVAL RATES. 2018.

2. National Cancer Institute, Cancer Stat Facts: Female Breast Cancer. 2017.

3. Breast Cancer Research Foundation, Emerging Therapies in Metastatic Breast Cancer. 2017.

4. van 't Veer, L.J., et al., Gene expression profiling predicts clinical outcome of breast cancer. Nature, 2002. 415: p. 530.

5. $\quad$ van de Vijver, M.J., et al., A Gene-Expression Signature as a Predictor of Survival in Breast Cancer. New England Journal of Medicine, 2002. 347(25): p. 1999-2009.

6. Pantel, K. and R.H. Brakenhoff, Dissecting the metastatic cascade. Nature Reviews Cancer, 2004. 4: p. 448.

7. Hanahan, D. and Robert A. Weinberg, Hallmarks of Cancer: The Next Generation. Cell, 2011. 144(5): p. 646-674.

8. Jin, X. and P. Mu, Targeting Breast Cancer Metastasis. Breast Cancer : Basic and Clinical Research, 2015. 9(Suppl 1): p. 23-34.

9. Ribatti, D., G. Mangialardi, and A. Vacca, Stephen Paget and the 'seed and soil' theory of metastatic dissemination. Clinical and Experimental Medicine, 2006. 6(4): p. 145-149.

10. Gupta, G.P. and J. Massagué, Cancer Metastasis: Building a Framework. Cell, 2006. 127(4): p. 679-695.

11. Wei, W. and M.T. Lewis, IDENTIFYING AND TARGETING TUMORINITIATING CELLS IN THE TREATMENT OF BREAST CANCER. Endocrinerelated cancer, 2015. 22(3): p. R135-R155.

12. Hirsch, D.S., Y. Shen, and W.J. Wu, Growth and Motility Inhibition of Breast Cancer Cells by Epidermal Growth Factor Receptor Degradation Is Correlated with Inactivation of Cdc42. Cancer Research, 2006. 66(7): p. 3523.

13. Wang, M., et al., Mechanism of immune evasion in breast cancer. OncoTargets and therapy, 2017. 10: p. 1561-1573. 
14. Eccles, S.A. and D.R. Welch, Metastasis: recent discoveries and novel treatment strategies. Lancet, 2007. 369(9574): p. 1742-1757.

15. Yu, Y., et al., Cancer-associated fibroblasts induce epithelial-mesenchymal transition of breast cancer cells through paracrine TGF- $\beta$ signalling. British Journal Of Cancer, 2013. 110: p. 724.

16. Izumi, D., et al., CXCL12/CXCR4 activation by cancer-associated fibroblasts promotes integrin $\beta 1$ clustering and invasiveness in gastric cancer. International Journal of Cancer, 2015. 138(5): p. 1207-1219.

17. Fidler, I.J., The pathogenesis of cancer metastasis: the \&\#39;seed and soil\&\#39; hypothesis revisited. Nature Reviews Cancer, 2003. 3: p. 453.

18. Palmer, G.M., et al., Optical imaging of tumor hypoxia dynamics. Journal of Biomedical Optics, 2010. 15(6): p. 066021.

19. Muz, B., et al., The role of hypoxia in cancer progression, angiogenesis, metastasis, and resistance to therapy. Hypoxia, 2015. 3: p. 83-92.

20. McKeown, S.R., Defining normoxia, physoxia and hypoxia in tumoursimplications for treatment response. The British Journal of Radiology, 2014. 87(1035): p. 20130676.

21. Vaupel, P., A. Mayer, and M. Höckel, Tumor Hypoxia and Malignant Progression, in Methods in Enzymology. 2004, Academic Press. p. 335-354.

22. Cronin, P.A., J.H. Wang, and H.P. Redmond, Hypoxia increases the metastatic ability of breast cancer cells via upregulation of CXCR4. BMC Cancer, 2010. 10(1): p. 225.

23. Ebbesen, P., et al., Taking advantage of tumor cell adaptations to hypoxia for developing new tumor markers and treatment strategies. Journal of Enzyme Inhibition and Medicinal Chemistry, 2009. 24(sup1): p. 1-39.

24. Rundqvist, H. and R.S. Johnson, Tumour oxygenation: implications for breast cancer prognosis. Journal of Internal Medicine, 2013. 274(2): p. 105-112.

25. Padua, D., et al., TGF $\beta$ primes breast tumors for lung metastasis seeding through angiopoietin-like 4. Cell, 2008. 133(1): p. 66-77.

26. Rankin, E.B. and A.J. Giaccia, Hypoxic control of metastasis. Science (New York, N.Y.), 2016. 352(6282): p. 175-180.

27. Xiao, Q. and G. Ge, Lysyl Oxidase, Extracellular Matrix Remodeling and Cancer Metastasis. Cancer Microenvironment, 2012. 5(3): p. 261-273. 
28. Lu, P., V.M. Weaver, and Z. Werb, The extracellular matrix: A dynamic niche in cancer progression. The Journal of Cell Biology, 2012. 196(4): p. 395.

29. Gilkes, D.M., G.L. Semenza, and D. Wirtz, Hypoxia and the extracellular matrix: drivers of tumour metastasis. Nature reviews. Cancer, 2014. 14(6): p. 430-439.

30. Alvaro, M.-H., et al., HIF-1\&\#945; Modulates Energy Metabolism in Cancer Cells by Inducing Over-Expression of Specific Glycolytic Isoforms. Mini-Reviews in Medicinal Chemistry, 2009. 9(9): p. 1084-1101.

31. Cui, X.-g., et al., HIF 1/2 $\alpha$ mediates hypoxia-induced LDHA expression in human pancreatic cancer cells. Oncotarget, 2017. 8(15): p. 24840-24852.

32. Kato, Y., et al., Cancer metabolism: New insights into classic characteristics. Japanese Dental Science Review, 2018. 54(1): p. 8-21.

33. Eales, K.L., K.E.R. Hollinshead, and D.A. Tennant, Hypoxia and metabolic adaptation of cancer cells. Oncogenesis, 2016. 5: p. e190.

34. Kim, J.-w., et al., HIF-1-mediated expression of pyruvate dehydrogenase kinase: A metabolic switch required for cellular adaptation to hypoxia. Cell Metabolism, 2006. 3(3): p. 177-185.

35. Buchheit, C.L., K.J. Weigel, and Z.T. Schafer, Cancer cell survival during detachment from the ECM: multiple barriers to tumour progression. Nature Reviews Cancer, 2014. 14: p. 632.

36. Miyazaki, K., et al., Identification of Functional Hypoxia Response Elements in the Promoter Region of the DEC1 and DEC2 Genes. Journal of Biological Chemistry, 2002. 277(49): p. 47014-47021.

37. Baba, K., et al., Hypoxia-induced ANGPTL4 sustains tumour growth and anoikis resistance through different mechanisms in scirrhous gastric cancer cell lines. Scientific Reports, 2017. 7(1): p. 11127.

38. Whelan, K.A., et al., Hypoxia Suppression of Bim and Bmf Blocks Anoikis and Luminal Clearing during Mammary Morphogenesis. Molecular Biology of the Cell, 2010. 21(22): p. 3829-3837.

39. Lee, S.-H., et al., HIF-1 is induced via EGFR activation and mediates resistance to anoikis-like cell death under lipid rafts/caveolae-disrupting stress. Carcinogenesis, 2009. 30(12): p. 1997-2004.

40. Heerboth, S., et al., EMT and tumor metastasis. Clinical and Translational Medicine, 2015. 4: p. 6. 
41. Smith, H.A. and Y. Kang, The Metastasis-Promoting Roles of Tumor-Associated Immune Cells. Journal of molecular medicine (Berlin, Germany), 2013. 91(4): p. 411-429.

42. Gray, L.H., et al., The Concentration of Oxygen Dissolved in Tissues at the Time of Irradiation as a Factor in Radiotherapy. The British Journal of Radiology, 1953. 26(312): p. 638-648.

43. Yang, M., et al., Prolyl hydroxylase domain enzymes: important regulators of cancer metabolism. Hypoxia, 2014. 2: p. 127-142.

44. Chan, D.A., et al., Coordinate Regulation of the Oxygen-Dependent Degradation Domains of Hypoxia-Inducible Factor 1 $\alpha$. Molecular and Cellular Biology, 2005. 25(15): p. 6415-6426.

45. Ziello, J.E., I.S. Jovin, and Y. Huang, Hypoxia-Inducible Factor (HIF)-1 Regulatory Pathway and its Potential for Therapeutic Intervention in Malignancy and Ischemia. The Yale Journal of Biology and Medicine, 2007. 80(2): p. 51-60.

46. Poillet-Perez, L., et al., Interplay between ROS and autophagy in cancer cells, from tumor initiation to cancer therapy. Redox Biology, 2015. 4: p. 184-192.

47. Rey, S., et al., Molecular targeting of hypoxia in radiotherapy. Advanced Drug Delivery Reviews, 2017. 109(Supplement C): p. 45-62.

48. Dengler, V.L., M. Galbraith, and J.M. Espinosa, Transcriptional Regulation by Hypoxia Inducible Factors. Critical reviews in biochemistry and molecular biology, 2014. 49(1): p. 1-15.

49. Semenza, G.L., The hypoxic tumor microenvironment: A driving force for breast cancer progression. Biochimica et Biophysica Acta (BBA) - Molecular Cell Research, 2016. 1863(3): p. 382-391.

50. Liu, Z.-j., G.L. Semenza, and H.-f. Zhang, Hypoxia-inducible factor 1 and breast cancer metastasis. Journal of Zhejiang University. Science. B, 2015. 16(1): p. 3243.

51. Clara Carlos, A., et al., Angiogenesis and expression of PDGF-C, VEGF, CD105 and HIF-1 $\alpha$ in human glioblastoma. Neuropathology, 2014. 34(4): p. 343-352.

52. Du, J., et al., PI3K and ERK-Induced Racl Activation Mediates Hypoxia-Induced HIF-1 $\alpha$ Expression in MCF-7 Breast Cancer Cells. PLOS ONE, 2011. 6(9): p. e25213. 
53. Guo, M., et al., Hypoxia Promotes Migration and Induces CXCR4 Expression via HIF-1 a Activation in Human Osteosarcoma. PLoS ONE, 2014. 9(3): p. e90518.

54. Wilson, S.S., et al., Expression of angiopoietin-like protein 4 at the fracture site: Regulation by hypoxia and osteoblastic differentiation. J Orthop Res, 2015. 33(9): p. 1364-73.

55. Liu, J.L., et al., [Mechanism of lysyl oxidase (LOX) in breast cancer invasion and metastasis]. Zhonghua Yi Xue Za Zhi, 2012.92(20): p. 1379-83.

56. Schito, L. and S. Rey, Hypoxic pathobiology of breast cancer metastasis. Biochimica et Biophysica Acta (BBA) - Reviews on Cancer, 2017. 1868(1): p. 239-245.

57. Masoud, G.N. and W. Li, HIF-1alpha pathway: role, regulation and intervention for cancer therapy. Acta Pharm Sin B, 2015. 5(5): p. 378-89.

58. Kenneth, N.S., et al., SWI/SNF Regulates the Cellular Response to Hypoxia. Journal of Biological Chemistry, 2009. 284(7): p. 4123-4131.

59. Melvin, A., S. Mudie, and S. Rocha, The chromatin remodeler ISWI regulates the cellular response to hypoxia: role of FIH. Molecular Biology of the Cell, 2011. 22(21): p. 4171-4181.

60. Ebert, B.L. and H.F. Bunn, Regulation of Transcription by Hypoxia Requires a Multiprotein Complex That Includes Hypoxia-Inducible Factor 1, an Adjacent Transcription Factor, and p300/CREB Binding Protein. Molecular and Cellular Biology, 1998. 18(7): p. 4089-4096.

61. Firth, J.D., B.L. Ebert, and P.J. Ratcliffe, Hypoxic Regulation of Lactate Dehydrogenase A: INTERACTION BETWEEN HYPOXIA-INDUCIBLE FACTOR 1 AND cAMP RESPONSE ELEMENTS. Journal of Biological Chemistry, 1995. 270(36): p. 21021-21027.

62. Ma, W., et al., Hypoxia-induced overexpression of DEC1 is regulated by HIFlalpha in hepatocellular carcinoma. Oncol Rep, 2013. 30(6): p. 2957-62.

63. Nebert, D.W., Transcription factors and cancer: an overview. Toxicology, 2002. 181-182: p. 131-141.

64. Bhagwat, A.S. and C.R. Vakoc, Targeting Transcription Factors in Cancer. Trends in Cancer. 1(1): p. 53-65.

65. Baxter, E., et al., Epigenetic regulation in cancer progression. Cell \& Bioscience, 2014. 4: p. 45-45. 
66. Gene Cards (Human Gene Database), BHLHE40 gene.

67. Yao, F., et al., Identification of Gene-Expression Signatures and Protein Markers for Breast Cancer Grading and Staging. PLOS ONE, 2015. 10(9): p. e0138213.

68. Sato, F., et al., DEC1 and DEC2 Crosstalk between Circadian Rhythm and Tumor Progression. Journal of Cancer, 2016. 7(2): p. 153-159.

69. Liu, Y., et al., DEC1 is positively associated with the malignant phenotype of invasive breast cancers and negatively correlated with the expression of claudin1. Int J Mol Med, 2013. 31(4): p. 855-60.

70. $\quad \mathrm{Bi}, \mathrm{H}$., et al., DEC1 regulates breast cancer cell proliferation by stabilizing cyclin E protein and delays the progression of cell cycle S phase. Cell Death \& Disease, 2015. 6(9): p. e1891.

71. Sato, F., et al., DEC1 and DEC2 Crosstalk between Circadian Rhythm and Tumor Progression. J Cancer, 2016. 7(2): p. 153-9.

72. Salminen, A., K. Kaarniranta, and A. Kauppinen, Hypoxia-Inducible Histone Lysine Demethylases: Impact on the Aging Process and Age-Related Diseases. Aging and Disease, 2016. 7(2): p. 180-200.

73. Ramadoss, S., X. Chen, and C.-Y. Wang, Histone Demethylase KDM6B Promotes Epithelial-Mesenchymal Transition. The Journal of Biological Chemistry, 2012. 287(53): p. 44508-44517.

74. Salton, M., T.C. Voss, and T. Misteli, Identification by high-throughput imaging of the histone methyltransferase EHMT2 as an epigenetic regulator of VEGFA alternative splicing. Nucleic Acids Research, 2014. 42(22): p. 13662-13673.

75. NCBI, EHMT2 euchromatic histone lysine methyltransferase 2 [ Homo sapiens (human) ]. 2018.

76. Cho, H.S., et al., Enhanced expression of EHMT2 is involved in the proliferation of cancer cells through negative regulation of SIAH1. Neoplasia, 2011. 13(8): $\mathrm{p}$. 676-84.

77. Wikipedia, Chromatin. 2018.

78. Melvin, A. and S. Rocha, Chromatin as an oxygen sensor and active player in the hypoxia response. Cellular Signalling, 2012. 24(1): p. 35-43.

79. Sena, J.A., L. Wang, and C.J. Hu, BRG1 and BRM chromatin-remodeling complexes regulate the hypoxia response by acting as coactivators for a subset of 
hypoxia-inducible transcription factor target genes. Mol Cell Biol, 2013. 33(19): p. 3849-63.

80. Jubierre, L., et al., BRG1/SMARCA4 is essential for neuroblastoma cell viability through modulation of cell death and survival pathways. Oncogene, 2016. 35(39): p. 5179-5190.

81. Do, S.I., et al., Increased Brahma-related Gene 1 Expression Predicts Distant Metastasis and Shorter Survival in Patients with Invasive Ductal Carcinoma of the Breast. Anticancer Res, 2016. 36(9): p. 4873-82.

82. Sokol, E.S., et al., SMARCE1 is required for the invasive progression of in situ cancers. Proceedings of the National Academy of Sciences, 2017. 114(16): p. 4153-4158.

83. Krutilina, R., et al., MicroRNA-18a inhibits hypoxia-inducible factor 1alpha activity and lung metastasis in basal breast cancers. Breast Cancer Res, 2014. 16(4): p. R78.

84. Society, A.C., Cancer Facts \& Figures 2016. http://www.cancer.org/research/cancerfactsstatistics/cancerfactsfigures2016/, 2016.

85. Kim, Y.N., et al., Anoikis resistance: an essential prerequisite for tumor metastasis. Int J Cell Biol, 2012. 2012: p. 306879.

86. Labelle, M. and R.O. Hynes, The initial hours of metastasis: the importance of cooperative host-tumor cell interactions during hematogenous dissemination. Cancer Discov, 2012. 2(12): p. 1091-9.

87. Nair, S.S. and R. Kumar, Chromatin remodeling in cancer: a gateway to regulate gene transcription. Mol Oncol, 2012. 6(6): p. 611-9.

88. Kadoch, C., et al., Proteomic and bioinformatic analysis of mammalian SWI/SNF complexes identifies extensive roles in human malignancy. Nat Genet, 2013. 45(6): p. 592-601.

89. Kiskinis, E., et al., Identification of BAF57 mutations in human breast cancer cell lines. Breast Cancer Res Treat, 2006. 98(2): p. 191-8.

90. Wilson, B.G., et al., Residual complexes containing SMARCA2 (BRM) underlie the oncogenic drive of SMARCA4 (BRG1) mutation. Mol Cell Biol, 2014. 34(6): p. 1136-44. 
91. Oike, T., et al., A synthetic lethality-based strategy to treat cancers harboring a genetic deficiency in the chromatin remodeling factor BRG1. Cancer Res, 2013. 73(17): p. 5508-18.

92. Hohmann, A.F. and C.R. Vakoc, A rationale to target the SWI/SNF complex for cancer therapy. Trends Genet, 2014. 30(8): p. 356-63.

93. Schiaffino-Ortega, S., et al., SWI/SNF proteins as targets in cancer therapy. J Hematol Oncol, 2014. 7(1): p. 81.

94. Davis, L.M., et al., Amplification patterns of three genomic regions predict distant recurrence in breast carcinoma. J Mol Diagn, 2007. 9(3): p. 327-36.

95. Sethuraman, A., et al., SMARCE1 regulates metastatic potential of breast cancer cells through the HIF 1A/PTK2 pathway. Breast Cancer Res, 2016. 18(1): p. 81.

96. Fan, M., et al., Comprehensive analysis of microRNA (miRNA) targets in breast cancer cells. J Biol Chem, 2013. 288(38): p. 27480-93.

97. Patsialou, A., et al., Selective gene-expression profiling of migratory tumor cells in vivo predicts clinical outcome in breast cancer patients. Breast Cancer Res, 2012. 14(5): p. R139.

98. Valastyan, S. and R.A. Weinberg, Tumor metastasis: molecular insights and evolving paradigms. Cell, 2011. 147(2): p. 275-92.

99. Garcia-Pedrero, J.M., et al., The SWI/SNF chromatin remodeling subunit BAF57 is a critical regulator of estrogen receptor function in breast cancer cells. J Biol Chem, 2006. 281(32): p. 22656-64.

100. Paoli, P., E. Giannoni, and P. Chiarugi, Anoikis molecular pathways and its role in cancer progression. Biochim Biophys Acta, 2013. 1833(12): p. 3481-98.

101. Guadamillas, M.C., A. Cerezo, and M.A. Del Pozo, Overcoming anoikis-pathways to anchorage-independent growth in cancer. J Cell Sci, 2011. 124(Pt 19): p. 3189-97.

102. Zouq, N.K., et al., FAK engages multiple pathways to maintain survival of fibroblasts and epithelia: differential roles for paxillin and p130Cas. J Cell Sci, 2009. 122(Pt 3): p. 357-67.

103. Kallergi, G., et al., Hypoxia-inducible factor-1alpha and vascular endothelial growth factor expression in circulating tumor cells of breast cancer patients. Breast Cancer Res, 2009. 11(6): p. R84. 
104. Consortium, E.P., An integrated encyclopedia of DNA elements in the human genome. Nature, 2012. 489(7414): p. 57-74.

105. Whelan, K.A., et al., The oncogene HER2/neu (ERBB2) requires the hypoxiainducible factor HIF-1 for mammary tumor growth and anoikis resistance. J Biol Chem, 2013. 288(22): p. 15865-77.

106. Kenneth, N.S., et al., SWI/SNF regulates the cellular response to hypoxia. J Biol Chem, 2009. 284(7): p. 4123-31.

107. Stampfer, M.R. and J.C. Bartley, Induction of transformation and continuous cell lines from normal human mammary epithelial cells after exposure to benzo[a]pyrene. Proc Natl Acad Sci U S A, 1985. 82(8): p. 2394-8.

108. Gyorffy, B., et al., An online survival analysis tool to rapidly assess the effect of 22,277 genes on breast cancer prognosis using microarray data of 1,809 patients. Breast Cancer Res Treat, 2010. 123(3): p. 725-31.

109. Cancer Genome Atlas, N., Comprehensive molecular portraits of human breast tumours. Nature, 2012. 490(7418): p. 61-70.

110. Wilson, B.G. and C.W. Roberts, SWI/SNF nucleosome remodellers and cancer. Nat Rev Cancer, 2011. 11(7): p. 481-92.

111. Kadoch, C. and G.R. Crabtree, Mammalian SWI/SNF chromatin remodeling complexes and cancer: Mechanistic insights gained from human genomics. Science Advances 2015. 1(5): p. e1500447.

112. Frisch, S.M. and H. Francis, Disruption of epithelial cell-matrix interactions induces apoptosis. J Cell Biol, 1994. 124(4): p. 619-26.

113. Mailleux, A.A., M. Overholtzer, and J.S. Brugge, Lumen formation during mammary epithelial morphogenesis: insights from in vitro and in vivo models. Cell Cycle, 2008. 7(1): p. 57-62.

114. Tancioni, I., et al., FAK activity protects nucleostemin in facilitating breast cancer spheroid and tumor growth. Breast Cancer Res, 2015. 17: p. 47.

115. Bouchard, V., et al., Fak/Src signaling in human intestinal epithelial cell survival and anoikis: differentiation state-specific uncoupling with the PI3-K/Akt-1 and MEK/Erk pathways. J Cell Physiol, 2007. 212(3): p. 717-28.

116. Zheng, Y., et al., Protein tyrosine kinase 6 protects cells from anoikis by directly phosphorylating focal adhesion kinase and activating AKT. Oncogene, 2013. 32(36): p. 4304-12. 
117. Cowley, G.S., et al., Parallel genome-scale loss of function screens in 216 cancer cell lines for the identification of context-specific genetic dependencies. Sci Data, 2014. 1: p. 140035.

118. Siegel, R.L., K.D. Miller, and A. Jemal, Cancer statistics, 2018. CA Cancer J Clin, 2018. 68(1): p. 7-30.

119. Gilkes, D.M., G.L. Semenza, and D. Wirtz, Hypoxia and the extracellular matrix: drivers of tumour metastasis. Nat Rev Cancer, 2014. 14(6): p. 430-9.

120. Rankin, E.B. and A.J. Giaccia, Hypoxic control of metastasis. Science, 2016. 352(6282): p. 175-80.

121. Petrova, V., et al., The hypoxic tumour microenvironment. Oncogenesis, 2018. 7(1): p. 10.

122. Sormendi, S. and B. Wielockx, Hypoxia Pathway Proteins As Central Mediators of Metabolism in the Tumor Cells and Their Microenvironment. Frontiers in Immunology, 2018. 9(40).

123. Weidle, H.U., et al., The Multiple Roles of Exosomes in Metastasis. Cancer Genomics \& Proteomics, 2017. 14(1): p. 1-16.

124. Harris, D.A., et al., Exosomes Released from Breast Cancer Carcinomas Stimulate Cell Movement. PLOS ONE, 2015. 10(3): p. e0117495.

125. O'Driscoll, L., Expanding on exosomes and ectosomes in cancer. N Engl J Med, 2015. 372(24): p. 2359-62.

126. Melo, S.A., et al., Cancer exosomes perform cell-independent microRNA biogenesis and promote tumorigenesis. Cancer Cell, 2014. 26(5): p. 707-21.

127. King, H.W., M.Z. Michael, and J.M. Gleadle, Hypoxic enhancement of exosome release by breast cancer cells. BMC Cancer, 2012. 12: p. 421.

128. Pawlus, M.R., L. Wang, and C.J. Hu, STAT3 and HIFlalpha cooperatively activate HIF1 target genes in MDA-MB-231 and RCC4 cells. Oncogene, 2014. 33(13): p. 1670-9.

129. Villar, D., et al., Cooperativity of stress-responsive transcription factors in core hypoxia-inducible factor binding regions. PLoS One, 2012. 7(9): p. e45708.

130. Miyazaki, K., et al., Identification of functional hypoxia response elements in the promoter region of the DEC1 and DEC2 genes. J Biol Chem, 2002. 277(49): p. 47014-21. 
131. Khurana, P., et al., HypoxiaDB: a database of hypoxia-regulated proteins. Database (Oxford), 2013. 2013: p. bat074.

132. Xiong, J., et al., The anti-metastatic effect of 8-MOP on hepatocellular carcinoma is potentiated by the down-regulation of bHLH transcription factor DEC1. Pharmacol Res, 2016. 105: p. 121-33.

133. Wu, Y., et al., The BHLH transcription factor DEC1 plays an important role in the epithelial-mesenchymal transition of pancreatic cancer. Int J Oncol, 2012. 41(4): p. 1337-46.

134. Chakrabarti, J., et al., The transcription factor DEC1 (stra13, SHARP2) is associated with the hypoxic response and high tumour grade in human breast cancers. Br J Cancer, 2004. 91(5): p. 954-8.

135. Nakashima, A., et al., DEC1 modulates the circadian phase of clock gene expression. Mol Cell Biol, 2008. 28(12): p. 4080-92.

136. Nishiyama, Y., et al., HIF-1alpha induction suppresses excessive lipid accumulation in alcoholic fatty liver in mice. J Hepatol, 2012. 56(2): p. 441-7.

137. Chung, S.Y., et al., Bhlhe40 Represses PGC-1alpha Activity on Metabolic Gene Promoters in Myogenic Cells. Mol Cell Biol, 2015. 35(14): p. 2518-29.

138. Kanda, M., et al., Transcriptional regulator Bhlhe40 works as a cofactor of T-bet in the regulation of IFN-gamma production in iNKT cells. Proc Natl Acad Sci U S A, 2016. 113(24): p. E3394-402.

139. Fan, M., et al., Comprehensive Analysis of MicroRNA (miRNA) Targets in Breast Cancer Cells. The Journal of Biological Chemistry, 2013. 288(38): p. 2748027493.

140. Fan, M., et al., Diverse gene expression and DNA methylation profiles correlate with differential adaptation of breast cancer cells to the antiestrogens tamoxifen and fulvestrant. Cancer Res, 2006. 66(24): p. 11954-66.

141. Patsialou, A., et al., Selective gene-expression profiling of migratory tumor cells in vivo predicts clinical outcome in breast cancer patients. Breast Cancer Research, 2012. 14(5): p. R139.

142. Whelan, K.A., et al., The Oncogene HER2/neu (ERBB2) Requires the Hypoxiainducible Factor HIF-1 for Mammary Tumor Growth and Anoikis Resistance. The Journal of Biological Chemistry, 2013. 288(22): p. 15865-15877.

143. Ciriello, G., et al., Comprehensive Molecular Portraits of Invasive Lobular Breast Cancer. Cell, 2015. 163(2): p. 506-19. 
144. Keerthikumar, S., et al., ExoCarta: A Web-Based Compendium of Exosomal Cargo. J Mol Biol, 2016. 428(4): p. 688-92.

145. Yáñez-Mó, M., et al., Biological properties of extracellular vesicles and their physiological functions. Journal of Extracellular Vesicles, 2015. 4: p. 10.3402/jev.v4.27066.

146. Abcam, Extracellular vesicles: an introduction.

147. Prenzel, N., et al., EGF receptor transactivation by G-protein-coupled receptors requires metalloproteinase cleavage of proHB-EGF. Nature, 1999. 402(6764): p. 884-8.

148. Rubin, J.S., et al., A broad-spectrum human lung fibroblast-derived mitogen is a variant of hepatocyte growth factor. Proc Natl Acad Sci U S A, 1991. 88(2): p. 415-9.

149. Atretkhany, K.S.N., et al., Chemokines, cytokines and exosomes help tumors to shape inflammatory microenvironment. Pharmacology \& Therapeutics, 2016.

168: p. 98-112.

150. Kucharzewska, P., et al., Exosomes reflect the hypoxic status of glioma cells and mediate hypoxia-dependent activation of vascular cells during tumor development. Proc Natl Acad Sci U S A, 2013. 110(18): p. 7312-7.

151. Keerthikumar, S., et al., Proteogenomic analysis reveals exosomes are more oncogenic than ectosomes. Oncotarget, 2015. 6(17): p. 15375-96.

152. St-Pierre, B., et al., Stra13 homodimers repress transcription through class $B$ Ebox elements. J Biol Chem, 2002. 277(48): p. 46544-51.

153. Bhawal, U.K., et al., Basic helix-loop-helix transcription factor DEC1 negatively regulates cyclin D1. The Journal of Pathology, 2011. 224(3): p. 420-429.

154. Seino, H., et al., Basic helix-loop-helix transcription factor DEC1 regulates the cisplatin-induced apoptotic pathway of human esophageal cancer cells. Biomed Res, 2015. 36(2): p. 89-96.

155. Bi, H., et al., DECl regulates breast cancer cell proliferation by stabilizing cyclin E protein and delays the progression of cell cycle $S$ phase. Cell Death Dis, 2015. 6: p. e1891.

156. Qian, Y., Y.S. Jung, and X. Chen, DeltaNp63, a target of DEC1 and histone deacetylase 2, modulates the efficacy of histone deacetylase inhibitors in growth 
suppression and keratinocyte differentiation. J Biol Chem, 2011. 286(14): p. 12033-41.

157. Li, Y., et al., The expression of antiapoptotic protein survivin is transcriptionally upregulated by DEC1 primarily through multiple sp1 binding sites in the proximal promoter. Oncogene, 2006. 25(23): p. 3296-306.

158. Martinez-Llordella, M., et al., CD28-inducible transcription factor DEC1 is required for efficient autoreactive CD4+ T cell response. J Exp Med, 2013. 210(8): p. 1603-19.

159. Chen, Y., et al., Tyrosine kinase receptor EGFR regulates the switch in cancer cells between cell survival and cell death induced by autophagy in hypoxia. Autophagy, 2016. 12(6): p. 1029-46.

160. Franovic, A., et al., Translational up-regulation of the EGFR by tumor hypoxia provides a nonmutational explanation for its overexpression in human cancer. Proc Natl Acad Sci U S A, 2007. 104(32): p. 13092-7.

161. Diaz, B., et al., Notch increases the shedding of HB-EGF by ADAM12 to potentiate invadopodia formation in hypoxia. J Cell Biol, 2013. 201(2): p. 279-92.

162. Wang, X.J., C.W. Feng, and M. Li, ADAM17 mediates hypoxia-induced drug resistance in hepatocellular carcinoma cells through activation of EGFR/PI3K/Akt pathway. Mol Cell Biochem, 2013. 380(1-2): p. 57-66.

163. Yotsumoto, F., et al., Molecular hierarchy of heparin-binding EGF-like growth factor-regulated angiogenesis in triple-negative breast cancer. Mol Cancer Res, 2013. 11(5): p. 506-17.

164. Tsai, Y.-P. and K.-J. Wu, Epigenetic regulation of hypoxia-responsive gene expression: Focusing on chromatin and DNA modifications. International Journal of Cancer, 2014. 134(2): p. 249-256.

165. Denko, N.C., et al., Investigating hypoxic tumor physiology through gene expression patterns. Oncogene, 2003. 22: p. 5907.

166. Doedens, A.L., et al., Hypoxia-inducible factors enhance the effector responses of CD8(+) T cells to persistent antigen. Nature immunology, 2013. 14(11): p. 11731182.

167. Gilkes, D.M. and G.L. Semenza, Role of hypoxia-inducible factors in breast cancer metastasis. Future oncology (London, England), 2013. 9(11): p. 16231636. 
168. Gerkes, E.H., et al., A heritable form of SMARCE1-related meningiomas with important implications for follow-up and family screening. Neurogenetics, 2016. 17(2): p. 83-9.

169. Raffalli-Ebezant, H., et al., Pediatric intracranial clear cell meningioma associated with a germline mutation of SMARCE1: a novel case. Childs Nerv Syst, 2015. 31(3): p. 441-7.

170. Smith, M.J., et al., SMARCE1 mutation screening in classification of clear cell meningiomas. Histopathology, 2017. 70(5): p. 814-820.

171. Yamaguchi, T., et al., Expression of BAF57 in ovarian cancer cells and drug sensitivity. Cancer Sci, 2015. 106(4): p. 359-66.

172. Brooks, D.L.P., et al., ITGA6 is directly regulated by hypoxia-inducible factors and enriches for cancer stem cell activity and invasion in metastatic breast cancer models. Molecular Cancer, 2016. 15: p. 26.

173. Link, K.A., et al., Targeting the BAF57 SWI/SNF subunit in prostate cancer: a novel platform to control androgen receptor activity. Cancer Res, 2008. 68(12): p. 4551-8.

174. Tai, Y.-L., L.-C. Chen, and T.-L. Shen, Emerging Roles of Focal Adhesion Kinase in Cancer. BioMed Research International, 2015. 2015: p. 13.

175. Yoon, H., et al., Understanding the Roles of FAK in Cancer: Inhibitors, Genetic Models, and New Insights. Journal of Histochemistry \& Cytochemistry, 2014. 63(2): p. 114-128.

176. Chan, K.T., C.L. Cortesio, and A. Huttenlocher, FAK alters invadopodia and focal adhesion composition and dynamics to regulate breast cancer invasion. The Journal of Cell Biology, 2009. 185(2): p. 357-370.

177. Zheng, Y., et al., The hypoxia-regulated transcription factor DEC1 (Stra13, SHARP-2) and its expression in gastric cancer. OMICS, 2009. 13(4): p. 301-6.

178. Talasila, K.M., et al., The angiogenic switch leads to a metabolic shift in human glioblastoma. Neuro Oncol, 2017. 19(3): p. 383-393.

179. Wang, W., et al., Hypoxia inducible BHLHB2 is a novel and independent prognostic marker in pancreatic ductal adenocarcinoma. Biochem Biophys Res Commun, 2010. 401(3): p. 422-8.

180. Li, Y., et al., Abundant expression of Dec1/stra13/sharp2 in colon carcinoma: its antagonizing role in serum deprivation-induced apoptosis and selective inhibition of procaspase activation. Biochem J, 2002. 367(Pt 2): p. 413-22. 
181. Zawel, L., et al., DEC1 is a downstream target of TGF-beta with sequencespecific transcriptional repressor activities. Proc Natl Acad Sci U S A, 2002. 99(5): p. 2848-53.

182. Ehata, S., et al., Transforming growth factor-beta promotes survival of mammary carcinoma cells through induction of antiapoptotic transcription factor DEC1. Cancer Res, 2007. 67(20): p. 9694-703.

183. Asanoma, K., et al., Regulation of the Mechanism of TWIST1 Transcription by BHLHE40 and BHLHE41 in Cancer Cells. Mol Cell Biol, 2015. 35(24): p. 4096109.

184. Lin, C.C., et al., Bhlhe40 controls cytokine production by T cells and is essential for pathogenicity in autoimmune neuroinflammation. Nat Commun, 2014. 5: p. 3551.

185. Zhou, Z.N., et al., Autocrine HBEGF expression promotes breast cancer intravasation, metastasis and macrophage-independent invasion in vivo. Oncogene, 2014. 33(29): p. 3784-3793.

186. De Luca, A., et al., The RAS/RAF/MEK/ERK and the PI3K/AKT signalling pathways: role in cancer pathogenesis and implications for therapeutic approaches. Expert Opinion on Therapeutic Targets, 2012. 16(sup2): p. S17-S27.

187. Masuda, H., et al., Role of Epidermal Growth Factor Receptor in Breast Cancer. Breast cancer research and treatment, 2012. 136(2): p. 10.1007/s10549-012-22899.

188. Yi, Y.W., et al., Inhibition of the PI3K/AKT pathway potentiates cytotoxicity of EGFR kinase inhibitors in triple-negative breast cancer cells. Journal of Cellular and Molecular Medicine, 2013. 17(5): p. 648-656.

189. Jeong, Y.-J., et al., Melittin suppresses EGF-induced cell motility and invasion by inhibiting PI3K/Akt/mTOR signaling pathway in breast cancer cells. Food and Chemical Toxicology, 2014. 68(Supplement C): p. 218-225.

190. Pachmayr, E., C. Treese, and U. Stein, Underlying Mechanisms for Distant Metastasis - Molecular Biology. Visceral Medicine, 2017. 33(1): p. 11-20.

191. Ranganathan, S., et al., Loss of EGFR-ASAP1 signaling in metastatic and unresectable hepatoblastoma. Scientific Reports, 2016. 6: p. 38347.

192. Minder, P., et al., EGFR Regulates the Development and Microarchitecture of Intratumoral Angiogenic Vasculature Capable of Sustaining Cancer Cell Intravasation. Neoplasia (New York, N.Y.), 2015. 17(8): p. 634-649. 


\section{VITA}

Aarti Sethuraman was born in 1986 in Tamil Nadu, India. She completed her BSc (2003-2006) and MSc (2006-2008) in Biotechnology from University of Mumbai. After graduating she worked at Yashraj Biotechnology, Navi Mumbai, India, from 2008-2010. She then worked as a Scientist at Biocon, Bangalore, India (2010-2011), before entering the Integrated Program in Biomedical Sciences (IPBS) Cancer and Developmental Biology track at the University of Tennessee Health Science Center in August 2012. Here she joined Dr. Meiyun Fan's laboratory studying the role of transcriptional regulators in metastatic breast cancer. She graduated with the degree Doctor of Philosophy in May 2018 with a focus on cancer biology.

\section{Google Scholar Profile:}

https://scholar.google.com/citations?user=yuf3FF4AAAAJ\&hl=en 